\title{
Pseudogap phenomena in ultracold atomic Fermi gases
}

\author{
Qijin Chen* and Jibiao Wang \\ Department of Physics and Zhejiang Institute of Modern Physics, \\ Zhejiang University, Hangzhou, Zhejiang 310027, China
}

(Dated: October 17, 2018)

\begin{abstract}
The pairing and superfluid phenomena in a two-component ultracold atomic Fermi gas is an analogue of Cooper pairing and superconductivity in an electron system, in particular, the high $T_{c}$ superconductors. Owing to the various tunable parameters that have been made accessible experimentally in recent years, atomic Fermi gases can be explored as a prototype or quantum simulator of superconductors. It is hoped that, utilizing such an analogy, the study of atomic Fermi gases may shed light to the mysteries of high $T_{c}$ superconductivity. One obstacle to the ultimate understanding of high $T_{c}$ superconductivity, from day one of its discovery, is the anomalous yet widespread pseudogap phenomena, for which a consensus is yet to be reached within the physics community, after over 27 years of intensive research efforts. In this article, we shall review the progress in the study of pseudogap phenomena in atomic Fermi gases in terms of both theoretical understanding and experimental observations. We show that there is strong, unambiguous evidence for the existence of a pseudogap in strongly interacting Fermi gases. In this context, we shall present a pairing fluctuation theory of the pseudogap physics and show that it is indeed a strong candidate theory for high $T_{c}$ superconductivity.
\end{abstract}

PACS numbers: 03.75.Ss, 03.75.Hh,67.85.Pq,74.25.Dw

Keywords: Pseudogap, Pairing fluctuation theory, atomic Fermi gases, BCS-BEC crossover, high $T_{c}$ superconductivity

\section{CONTENTS}

\section{Introduction}

II. What is a pseudogap? - Pseudogap phenomena in high $T_{c}$ superconductors

A. What is a pseudogap?

B. Pseudogap in the normal state above $T_{c}$

C. Pseudogap in the superfluid state below $T_{c}$

D. Theoretical debate about the nature of the pseudogap

III. Pairing fluctuation theory for the pseudogap

A. Various pairing fluctuation theories for BCS-BEC crossover

B. Pairing fluctuation theory for the pseudogap

C. Extended to Fermi gases in a trap

D. Thermodynamics and superfluid density

IV. Key results of the present pairing fluctuation theory 11

A. Two-component homogeneous Fermi gases in the 3D continuum

B. Application for the cuprates: quasi-2D superconductors on a lattice with a $d$-wave pairing symmetry

C. 3D Fermi gas in an isotropic trap

V. Experimental evidence of the pseudogap in atomic Fermi gases

A. Thermodynamics and density profiles

B. Momentum distribution

.

12

ses
Study of atomic Fermi gases, especially the pairing and
superfluid phenomena, has become a major field in physics research over the last decade $[1,2]$. Intrinsically a manybody system, atomic Fermi gases have attracted physicists from both condensed matter and atomic, molecular and optics (AMO) communities, as well as from other communities, e.g., nuclear and particle physics and astrophysics. Even superstring theorists have now found it a play ground for the ingenious idea of the AdS/CFT correspondence [3-6]. This is primarily due to the fact that many tunable parameters have been made accessible experimentally for atomic Fermi gases, including temperature, pairing interaction strength, pairing symmetry, population imbalance, mass imbalance, geometric aspect ratio of the trap, optical lattices, and dimensionality, etc., as well as extra degrees of freedom such as spin-orbit coupling and synthetic gauge fields, which make atomic gases 
a suitable system for quantum simulation and quantum engineering of existing and previously unknown systems, and have thus provided a great opportunity for studying many exotic quantum phenomena.

In terms of superfluidity, atomic Fermi gases can be thought of as the charge neutral counterpart of superconductors, which have been an important subject in contemporary condensed matter physics. In particular, high $T_{c}$ superconductivity has been a great challenge since its discovery a quarter century ago. With tunable interactions, it is strongly hoped that one may learn about the notoriously difficult problem of high $T_{c}$ superconductivity via studying atomic Fermi gases.

At the heart of the high $T_{c}$ problem is the widespread anomalous normal state gap [7] in the single particle excitation spectrum, which has been referred to as the pseudogap, and has emerged since the discovery of high $T_{c}$ superconductors. It is essential to understand the pseudogap phenomena in order to reach a consensus on the mechanism of high $T_{c}$ superconductivity. Due to the analogy between superfluidity and superconductivity, it is expected that study of the pairing and superfluid phenomena in ultracold Fermi gases may eventually shed light on the pseudogap physics and thus the mechanism of high $T_{c}$ superconductivity.

The first and most widely explored parameter in ultracold atomic Fermi gases is the pairing interaction strength. Using an $s$-wave Feshbach resonance, one can tune the effective pairing strength from the weak coupling limit of Bardeen-Cooper-Schrieffer (BCS) superfluidity [8] all the way through the strong coupling limit of Bose-Einstein condensation (BEC) [9-12]. In this way, the theoretical idea of BCS-BEC crossover, which was first proposed by Eagles [13] and Leggett [14] at zero temperature $T$ and then extended to finite $T$ by Nozieres and Schmitt-Rink [15] and many others [16-36], can be realized and studied systematically in experiment.

There have been a few milestones in experimental studies of the superfluidity and BCS-BEC crossover of ultracold Fermi gases. Degenerate Fermi gases was achieved a few years [37] after the experimental realization of BEC of dilute gases of bosonic alkali atoms [38-41], such as ${ }^{23} \mathrm{Na},{ }^{87} \mathrm{Rb}$, and ${ }^{7} \mathrm{Li}$. BEC of diatomic molecules on the BEC side of a Feshbach resonance was first reported in 2003 in Fermi gases of ${ }^{40} \mathrm{~K}$ and of ${ }^{6} \mathrm{Li}$ [42-44]. Superfluidity in a Fermi gas in the entire BCS-BEC crossover was achieved and reported in 2004 [45-48]. A continuous thermodynamic superfluid transition was not observed until late 2004 [49]. A smoking gun of superfluidity came from the Ketterle group in 2005 which reported observation of vortex lattices, a macroscopic manifestation of quantum phenomena, from the BCS through BEC regimes [50]. Population (or spin) imbalance has been the second experimental parameter which has been explored in ultracold Fermi gases since 2006 [51, 52]. It is expected to lead to new phases such as phase separation and the exotic FuldeFerrell-Larkin-Ovchinnikov (FFLO) states [53-55]. Further parameters which have been gradually explored experimentally include geometric aspect ratio and dimensionality, mass imbalance, pairing symmetry such as $p$-wave, synthetic gauge fields and spin-orbit coupling, long range interactions as in dipolar molecules and magnetic atoms, as well as periodic potential, i.e., optical lattices.

There have been a few reviews on the subject of atomic Fermi gases. References [1] and [57] are the earliest reviews on this subject, emphasizing the similarity between Fermi gases and high $T_{c}$ superconductivity as well as BCS-BEC crossover physics. Reference [58] reviewed the progress on the physics of degenerate Fermi gases from the theoretical perspective. Strong correlation effects in terms of many-body physics were only quickly mentioned as "other theoretical approaches". The review by Chin et al [59] focuses more on Feshbach resonances, with a very brief touch on the experiments on BCS-BEC crossover. A few papers in the Varenna proceedings [60], as well as Ref. [2], also gave an review on the experimental and theoretical progress on atomic Fermi gases, without much emphasis on the pseudogap physics. It is the purpose of the current paper to give a more or less systematic review on the study of the pseudogap physics in cold atomic Fermi gases.

The rest of this paper is arranged as follows. In Sec. II, we shall first introduce the concept of pseudogap in the context of high $T_{c}$ superconductivity, and then provide examples of the pseudogap phenomena above and below $T_{c}$, and finally give an overview of the theoretical debate on the nature of the pseudogap. In Sec. III, we shall start by a summary of various theories of pairing fluctuations in the context of BCS-BEC crossover, and then present a particular pairing fluctuation theory for the pseudogap phenomena for a homogeneous system and later extend to Fermi gases in a trap. We shall end this section by presenting theoretical results on the thermodynamics and superfluid density. In Sec. IV, we shall show key results from the present pairing fluctuation theory on the pseudogap phenomena in both the 3D homogeneous case (Subsec. IVA) and in a trap (Subsec. IVC). In Subsec. IVB, we shall also give a summary of the applications of the present theory to high $T_{c}$ superconductors with a $d$-wave pairing symmetry. In Sec. V, we will present a series of experiments which show strong evidence or support of a pseudogap in unitary Fermi gases. While we focus mainly on population balanced two component Fermi gases, we shall show one case of population imbalanced Fermi gas experiment. In Sec. VI, we shall discuss the effect of particle-hole fluctuations and propose further experiments on pseudogap physics. Finally, we will conclude in Sec. VII.

\section{WHAT IS A PSEUDOGAP? - PSEUDOGAP PHENOMENA IN HIGH $T_{c}$ SUPERCONDUCTORS}

\section{A. What is a pseudogap?}

We begin by introducing the concept of pseudogap, which has emerged since day one of high $T_{c}$ superconductivity. In BCS theory, when the superconducting order parameter $\Delta$ becomes nonzero below the transition temperature $T_{c}$, a gap opens up at the Fermi level in the single particle excitation spectrum. The density of states (DOS) becomes zero within the gap. This gap originates purely from the order parameter 

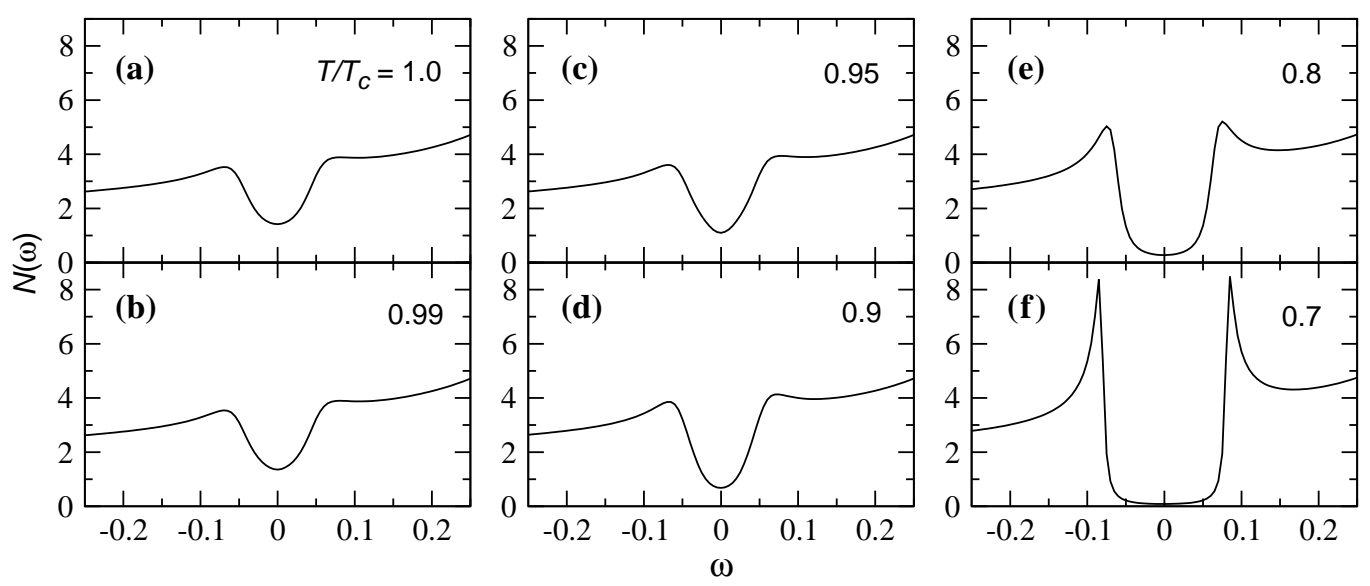

Figure 1. Typical evolution of the density of states in the presence of a pseudogap for an $s$-wave superconductor as a function of temperature, calculated for an quasi-2D superconductor on a square lattice at quarter filling. Panels (a)-(f) correspond to various temperatures (as labeled) decreasing from $T=T_{c}$. The DOS drops rapidly as the system enters the superconducting state below $T_{c}$. At $T / T_{c} \sim 0.7$, as shown in (f), the DOS is close to a true gap as that of strict BCS theory. The frequency $\omega$ is in units of the $2 \mathrm{D}$ half band width. Taken from Ref. [56].

and therefore vanishes at and above $T_{c}$. Soon after the high $T_{c}$ superconductivity was discovered in cuprates, an excitation gap was observed already above $T_{c}$, below a higher temperature $T^{*}$ (which is referred to as the pseudogap crossover temperature). Without phase coherence, such a gap does not lead to complete depletion of the DOS within the gap, but rather the DOS was only partially depleted. As $T$ approaches $T_{c}$ from above, the DOS drops quickly to zero at the Fermi level once phase coherence sets in as the system enters the superconducting state. In contrast to the true gap below $T_{c}$, the gap observed experimentally above $T_{c}$ has been referred to as a pseudogap. Whether the pseudogap persists below $T_{c}$ has been under debate.

The typical behavior of the DOS near the Fermi level for a pseudogapped superconductor is shown in Fig. I for various temperatures from $T_{c}$ to slightly below $T_{c}$. The curves are calculated theoretically for an $s$-wave superconductor on a quasitwo dimensional (2D) lattice. From Fig. I(a), one can see clearly a partial depletion of the DOS around the Fermi level $(\omega=0)$. As $T$ lowers into the broken symmetry state, phase coherence sets in, and the DOS drops rapidly. At $T=0.7 T_{c}$, the depletion within the gap becomes almost complete so that the DOS looks like one in a strict BCS mean-field theory, with two sharp coherent peaks at the gap edges.

\section{B. Pseudogap in the normal state above $T_{c}$}

Above $T_{c}$, the pseudogap manifests itself in various physical quantities, including the $d I / d V$ characteristics in tunneling spectroscopy [62-65], specific heat [66, 67], dc resistivity [68-70], nuclear magnetic resonance (NMR) [71-76], infrared and ac conductivity [77-79], neutron scattering [8082], Raman scattering [83-86], Nernst effect [87-90], spin susceptibility, etc., as a function of temperature. The most direct probe, of course, is the angle-resolved photoemission spectroscopy (ARPES) [61, 91, 92], which probes directly the spectral function $A(\mathbf{k}, \omega)$. Along the Fermi surface, the quasiparticle coherence peak position in the measured spectral function reveals directly the gap parameter. A review on various experiments on the pseudogap phenomena can be found in Ref. [7]. Here we only show a couple of examples to illustrate the pseudogap phenomena.

High $T_{c}$ superconductors of the cuprates, such as $\mathrm{YBa}_{2} \mathrm{Cu}_{3} \mathrm{O}_{7-\delta}$ (YBCO), $\mathrm{Bi}_{2} \mathrm{Sr}_{2} \mathrm{CaCu}_{2} \mathrm{O}_{8+\delta}$ (Bi2212) and $\mathrm{La}_{2-x} \mathrm{Sr}_{x} \mathrm{CuO}_{4}$ (LSCO), have a layered structure, with charge carriers moving in the copper-oxide planes. The electron transport along the $c$-axis (i.e., the direction perpendicular to the planes) is largely incoherent. This makes the cuprates quasi-2D materials. While the parent compounds are insulating antiferromagnets, superconductivity occurs at low $T$ upon hole doping [93]. Within the superconducting ( $a b$ )planes, it is now known that the order parameter $\Delta$ of the cuprate superconductors has a $d_{x^{-}} y^{2}$ symmetry, such that $\Delta=\Delta_{0}\left(\cos k_{x}-\cos k_{y}\right) / 2$, where we have set the in-plane lattice constant $a$ to unity. Thus the gap has a maximum in the anti-nodal directions near $(\pi, 0)$, whereas it closes in the nodal directions from $\Gamma$ to $(\pi, \pi)$ in the Brillouin zone (BZ). The order parameter changes sign across the nodal points along the Fermi surface.

Shown in Fig. 2(a) is a schematic phase diagram for the cuprate superconductors. The transition temperature $T_{c}$ reaches a maximum around doping concentration $x=0.155$. There is a temperature range between $T_{c}$ and $T^{*}$ in the underdoped regime where a finite pseudogap exists. Shown in Fig. 2(b) are the ARPES measurements of the excitation gap near $(\pi, 0)$ for Bi2212 at different doping concentrations. At optimal doping ( $T_{c}=87 \mathrm{~K}$ sample), the gap closes roughly at $T_{c}$, similar to that predicted in BCS theory. However, for the underdoped samples, it is clear that the gap persists at very high $T$. This is the most direct measurement, and hence evidence of the existence, of a pseudogap above $T_{c}$.

In Fig. 3, we present, as an example, typical normalinsulator-superconductor (SIN) tunneling spectra measured 

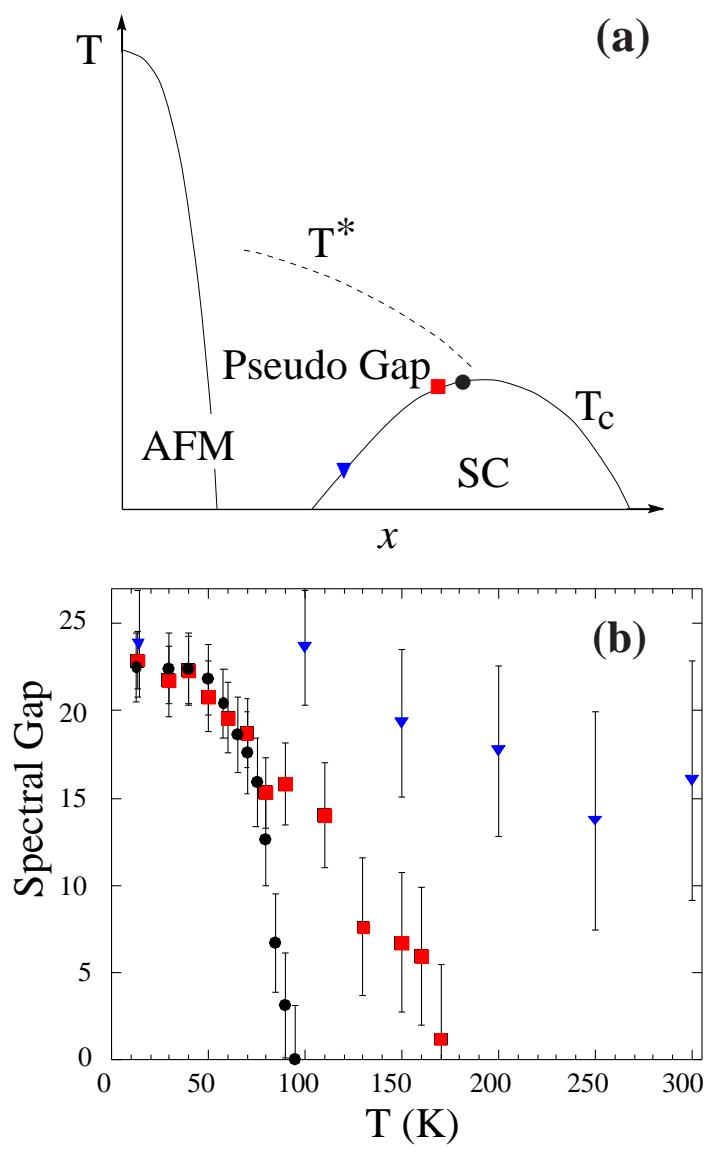

Figure 2. (a) Schematic phase diagram for the cuprate superconductors (The horizontal axis is the hole doping concentration), and (b) ARPES measurement of the temperature dependence of the excitation gap at $(\pi, 0)$ in a near-optimal $T_{c}=87 \mathrm{~K}$ sample $(\bullet)$, and underdoped $83 \mathrm{~K}(\boldsymbol{\square})$ and $10 \mathrm{~K}(\boldsymbol{\nabla})$ samples. The gap values were determined via leading edge shift from the Fermi level. The units for the gap are meV. Panel (b) is taken from Ref. [61].

for an underdoped cuprate superconductor as a function of temperature, using scanning tunneling microscopy (STM). Here the $d I / d V$ characteristics can be regarded as the DOS, but broadened by thermal effects. In sharp contrast with a BCS mean-field true gap, the pseudogap does not close even at $T^{*}$. But rather, the coherence peaks broaden and the DOS fills in with increasing $T$. At $T^{*}$, the sign of DOS depletion disappears and so does the pseudogap. The way the pseudogap disappears at high $T$ is a clear distinction from that of a true gap in a weak coupling BCS superconductor, which shrinks in magnitude to zero at $T_{c}$.

Similar behavior can be found in the ARPES energy distribution curves (EDC) for underdoped samples as well [61,91]. Usually, an ARPES EDC curve consists of a quasiparticle coherence peak on top of an incoherent background. At $T \ll T_{c}$, the coherence peak is sharp and pronounced. Once the temperature rises above $T_{c}$, for an underdoped sample, it becomes broadened quickly, and the spectral weight under the peak decreases with $T$ rapidly, until it merges with the large incoherent background. At the same time, the peak location al-

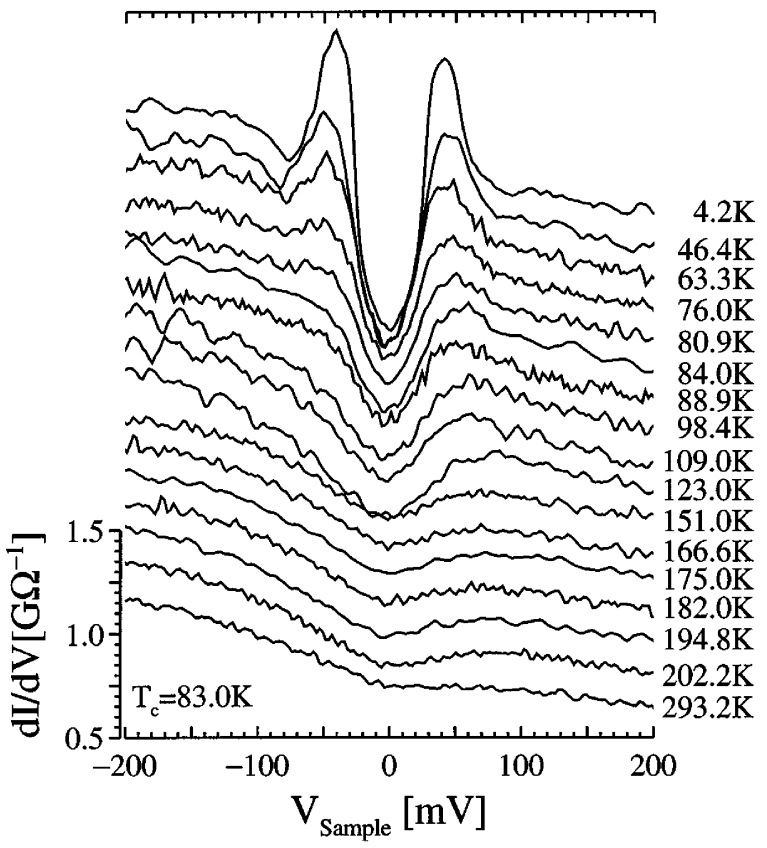

Figure 3. Typical tunneling spectra for an underdoped cuprate superconductor as a function of temperature. Shown data were measured on an underdoped Bi2212 sample of $T_{c}=83 \mathrm{~K}$. The horizontal axis is the bias. The conductance scale corresponds to the $293 \mathrm{~K}$ spectrum, the other spectra are offset vertically for clarity. Taken from Ref. [63].

most does not move with $T$. This can be seen in Fig. 4. The EDC curves in Figs. 4(a) and (b) were taken along the cut in the Brillouin zone shown Fig. 4(c), which goes across the Fermi level. Panels (a) and (b) correspond to low $T \ll T_{c}$ and above $T_{c}$ cases, respectively, with curves of the same $\mathbf{k}$ lined up together. As the cut goes through the Fermi surface, the coherence peak reaches the minimum quasiparticle excitation energy, as determined by the excitation gap $\Delta_{\mathbf{k}}$. It is obvious that the coherence peak in the pseudogap case is much broader and less pronounced than its superconducting counterpart.

\section{Pseudogap in the superfluid state below $T_{c}$}

Figures $2-4$ and most experimental measurements show clear evidence of the existence of a pseudogap at and above $T_{c}$. It is a natural question to ask how the pseudogap above $T_{c}$ and the superconducting gap below $T_{c}$ connect to each other at $T_{c}$. There have been intensive debates on this issue over the years. The answer to this important question depends on the interpretation of the pseudogap in different theories. Despite the differences from one theory to another, one can think of two possibilities in general. One possibility is that the pseudogap becomes the superconducting gap instantly once the system enters the superconducting state across $T_{c}$. The other is that the order parameter or the superconducting gap increases gradually from zero at $T_{c}$. For the former, one would see a first order phase transition and a jump in the order parameter and superfluid density across $T_{c}$. For the latter possibility, 


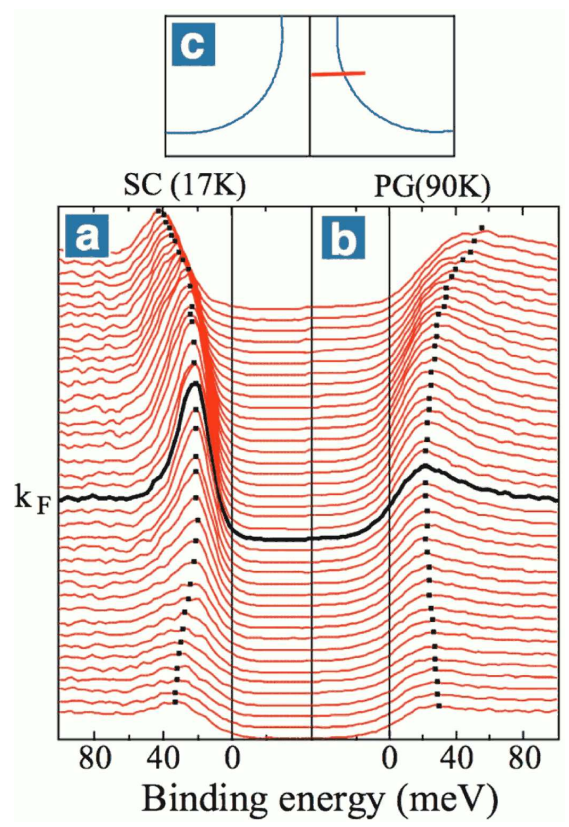

Figure 4. Comparison of EDCs between (a) the superconducting state $(T=17 \mathrm{~K})$ and $(\mathrm{b})$ the pseudogap phase $(T=90 \mathrm{~K})$ for a Bi2212 film with $T_{c}=80 \mathrm{~K}$ for the cut in the zone shown in (c). The thick curves in (a) and (b) correspond to where the cut goes through the Fermi surface. Here SC and PG denote superconducting and pseudogap state, respectively. Taken from Ref. [92].

the pseudogap necessarily persists into the superfluid state, in order to keep the total excitation gap smooth across $T_{c}$ as observed in ARPES data and other experiments. Given these rather obvious differences between these two possibilities, superfluid density $\left(n_{s} / m\right)$ or in-plane London penetration depth $(\lambda)$ measurements seem to have unambiguously ruled out the former possibility. Indeed, superfluid density $n_{s} / m \propto \lambda^{-2}$ vanishes continuously as $T$ approaches $T_{c}$ from below in bulk cuprate superconductors.

At a more concrete level, compatible with the first possibility may be a school of microscopic theories which consider the pseudogap above $T_{c}$ as a signature of a competing hidden order, such as the $d$-density wave (DDW) order [94], the staggered orbital current [95-97], loop current order [98, 99], etc. Given the hidden order assumption, a natural prediction would be that the hidden ordered phase gives way completely to the superconducting order across $T_{c}$, as in most other phase transitions. However, if this is true, not only a first order transition is necessary, but also it would take a miracle for the total excitation gap to remain so smooth across $T_{c}$ as observed experimentally. Then to pass the experimental test, these hidden order theories may also need to associate themselves with the second possibility, namely the hidden order parameter (and thus the pseudogap) survives the superconducting phase transition and coexists with the superconducting order below $T_{c}$. Together they contribute to the total excitation gap.

Among the second possibility, there are different scenarios which give rise to different interpretations and different temperature dependencies of the pseudogap. These differ-

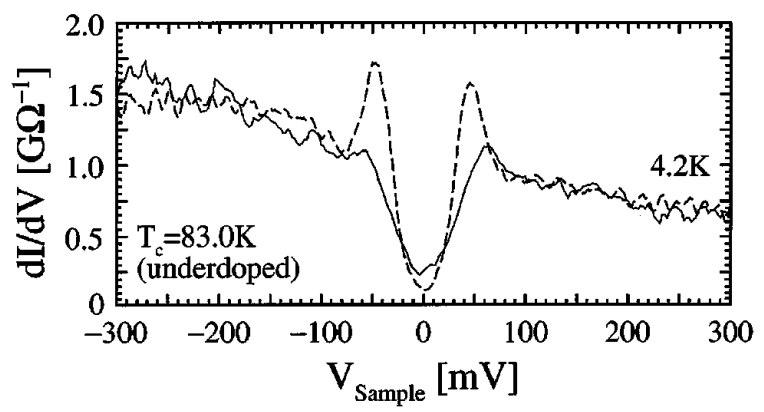

Figure 5. STM measurements of the $d I / d V$ characteristics of an underdoped Bi2212 sample inside (solid) and outside (dashed) a vortex core at low $T=4.2 \mathrm{~K}$. Here $T_{c}=83 \mathrm{~K} . d I / d V$ is proportional to the density of states. Taken from Ref. [62].

ences are associated with the origins of the pseudogap in these theories or conjectures. In order to fit the specific heat data for underdoped superconductors, Loram and coworkers $[66,100,101]$ contemplated that the pseudogap below $T_{c}$ may take its value at (and above) $T_{c}$ such that it is relatively temperature independent. As a consequence, (the magnitude of) the order parameter $\left(\Delta_{s c}\right)$ is much smaller than the total excitation gap $(\Delta)$ at all $T<T_{c}$ for an underdoped cuprate superconductor. We note that this was a rather simple recipe without any theoretical justification. Among microscopic theories, while the competing hidden order theories may be associated with the second possibility, a most natural school of theories in this category would be the precursor superconductivity, in which the pseudogap is a precursor to the superconductivity and originates from the same pairing as that causes the superconducting order at low $T$. We will elaborate further on this in the next subsection.

To probe the pseudogap below $T_{c}$, if it does exist, the best way is arguably to suppress the order parameter. Luckily, this can be achieved inside a vortex core. Figure 5 shows STM measurements of the $d I / d V$ characteristics of an underdoped Bi2212 sample inside and outside a vortex core at very low $T$. Outside the vortex core, the order parameter is large at low $T$, and there are sharp peaks at the gap edges (dashed curve). At the center of the vortex core, the superconducting order parameter is suppressed to zero (solid curve). Nevertheless, it is clear that the $d I / d V$ curve shows a strong depletion of the DOS within the peaks. The peak locations are roughly the same as those outside the core. Of course, such a depletion is absent above the pseudogap crossover temperature $T^{*}$. Therefore, this plot serves as evidence of pseudogap below $T_{c}$ in an underdoped cuprate. Indeed, this can be naturally explained within a pairing fluctuation theory [23]; In addition to noncondensed pairs, the magnetic field inside the vortex core causes the originally condensed pairs lose phase coherence and thus contribute to the pseudogap rather than the order parameter [102]. On the other hand, as one may notice, in order for the competing hidden order theories to explain the survival of the total gap at the center of the vortex core at very low $T$, one would have to assume that the superconducting order is converted into hidden order parameter by the magnetic field. 
This, however, is very unnatural.

\section{Theoretical debate about the nature of the pseudogap}

The pseudogap phenomena is widespread in high $T_{c}$ superconductivity experiments. However, a consensus of its origin is yet to be reached. There have been many different theories attempting to explain the nature of the pseudogap. Most of these theories only provide qualitative pictures, incapable of quantitative calculations.

Early models include the resonating valence bond (RVB) theory of Anderson [103, 104] and the closely related spincharge separation idea $[105,106]$. In these theories, the pseudogap originates from the spin gap of the antiferromagnetic spin pairing (i.e., the spinon pairing). Despite the well established phenomena of spin-charge separation in 1D, so far there has been no experimental support for spin-charge separation in $2 \mathrm{D}$, not to mention 3D.

About the same time, Uemura and coworkers [107, 108] noticed possible connections between the cuprate superconductivity and BEC via the well-known Uemura plot of $T_{c}$ versus superfluid density $n_{s} / m^{*}$. This has been used to suggest that the cuprates have to do with BCS-BEC crossover. In an attempt to explain the pseudogap phenomena, Lee and coauthors $[16,17]$ proposed a boson-fermion model. The pseudogap phenomena was then explored using the BCS-BEC crossover idea in 3D continuum [18-22] or the negative- $U$ Hubbard model on a lattice [26-29], assuming an $s$-wave pairing symmetry. These theories belong to the school of precursor superconductivity, in which the pseudogap above $T_{c}$ and the superconducting gap below $T_{c}$ originate from the same pairing interaction, and thus the pseudogap in the normal state is a precursor to superconductivity below $T_{c}$. A theory of the broken symmetry state and the presence of the pseudogap below $T_{c}$, especially for $d$-wave pairing, was not available until the work of Refs. [23-25].

It is worth mentioning that in a very recent work [109], Mishra and coworkers showed that a pseudogap which is not associated with pairing would suppress $T_{c}$ to zero. Therefore, they concluded that the pseudogap observed in the cuprates must be due to pairing.

Compatible with but distinct from the precursor superconductivity school are theories based on phase fluctuations such as that of Emery and Kivelson [110] and the QED3 theory of Tesanovic and coworkers [111]. The former addresses mainly spin-wave type of phase fluctuations whereas the latter has an emphasis on vortex fluctuations. In both theories, the pseudogap originates from a pairing field without phase coherence; the pairing field emerges at $T^{*}$ but the phase coherence does not lock in until a lower temperature $T_{c}$. The strong Nernst signals observed above $T_{c}$ in underdoped cuprates [87-89] may be regarded as a support for the latter theory. On the other hand, it should be noted that the Nernst effect data can be explained within a pairing fluctuation theory as well $[90,112]$.

Both the spin gap scenarios as in RVB and spin-charge separation and charge gap scenarios as in precursor superconductivity and phase fluctuation pictures have to do with pairing in the particle-particle channel. A big departure from this common feature are the competing hidden order ideas, mentioned in Subsec. II C, which take the pseudogap as a hidden order parameter. For example, the DDW order is associated with the particle-hole channel. The staggered current and loop current order are not related to pairing, either. They rely on the underlying quasi-2D lattice structure of the cuprates.

The RVB and spin-charge separation ideas can be traced back to the fact that the parent compounds of the cuprate superconductors are insulating antiferromagnets in the Mott state, with an underlying quasi-2D, layered lattice structure. The DDW, staggered current and loop current ideas have also to do with the underlying lattice structures, which are apparently not pertinent to the atomic Fermi gases in a big single trap. Deeper than but closely related to the pseudogap phenomena is the mechanism of superconductivity in the cuprates, namely, what provides the glue for the electrons to pair up.

Luckily, for atomic Fermi gases, the underlying pairing interaction is known and can be precisely manipulated experimentally. While one may continue to debate on the origin of the pseudogap phenomena in the cuprates, as far as the atomic Fermi gases are concerned, this fact does make the pairing fluctuation theory the most natural candidate for the theory of the superfluidity and pairing.

\section{PAIRING FLUCTUATION THEORY FOR THE PSEUDOGAP}

\section{A. Various pairing fluctuation theories for BCS-BEC crossover}

Pairing fluctuation theories belong to the school of precursor superfluidity. There are different pairing fluctuation theories. Nevertheless, common to these theories are strong pairing fluctuations or pairing correlations already above $T_{c}$, which necessarily cause deviation of the system behavior from those described by the BCS mean-field theory. The first thing that has been looked into is the superfluid transition temperature $T_{c}$. Not all of these theories contain a pseudogap in their single particle excitation spectrum, nor are they all selfconsistent. As the pairing strength varies, a pairing fluctuation theory is often used to address the BCS-BEC crossover problem, and thus is often referred to as a BCS-BEC crossover theory as well. Note that from this section on, we will use the term "superfluidity" in place of "superconductivity", in order to be appropriate for both superconductors and charge neutral superfluids.

The very first work on finite temperature BCS-BEC crossover, by Noziéres and Schmitt-Rink (NSR) [15] in 1985, can be regarded as the earliest pairing fluctuation theory. However, in the NSR theory, only bare Green's functions are involved so that the pairing fluctuations induced self energy does not feed back into the $T_{c}$ equation. As a consequence, pseudogap does not appear in the NSR theory. Although one may find features of pseudogap via further calculation of the spectral function with the self-energy included, this procedure 
certainly breaks self-consistency. Indeed, not including the self energy in the $T_{c}$ equation itself introduces inconsistency. For example, the $T_{c}$ equation is inconsistent with the condition

$$
\frac{\partial \Omega_{S}}{\partial \Delta}=0
$$

as $T_{c}$ is approached from below, where $\Omega_{S}$ is the thermodynamic potential in the superfluid state. Sa de Melo et al. [18] obtained identical equations as NSR, using a Saddle point approximation plus Gaussian fluctuations. There have been many studies in the literature using a similar approximation [19]. Milstein et al [113] used a similar treatment but within a two-channel model. Indeed, it has turned out that the saddle point approximation with Gaussian fluctuations and the NSR approximation are equivalent. With a narrow Feshbach resonance in a two-channel model, Ohashi and Griffin [114] caculated $T_{c}$ using the NSR approximation. Strinati and coworkers $[115,116]$ also followed the NSR calculation found the same $T_{c}$ and number equations as NSR. There are other pairing fluctuation theories on BCS-BEC crossover based on the NSR approximation. Noticeably, rather than fixing the inconsistency in the $T_{c}$ equation, $\mathrm{Hu}$ and Drummond [117] proposed to add an extra term in the number equation. This necessarily leads to two unphysical results: (i) This extra term does not exist above $T_{c}$, so that it will give rise to a different $T_{c}$ and chemical potential $\mu$, depending on whether $T_{c}$ is approached from above or below. (ii) In a trap, a uniform global chemical potential requires that the density jumps across the edge of the superfluid core. In fact, should the $T_{c}$ equation is fixed so that Eq. (1) is satisfied, this extra term would vanish automatically. More systematic and detailed comparison between the NSR-based theories and the pairing fluctuation theory which we will present soon below can be found in Ref. [118].

Using a $G G$ scheme for the $T$-matrix approximation, which is sometimes referred to as a conserving approximation, Haussmann [32] and Tchernyshyov [33] et al developed a different kind of pairing fluctuation theory, which leads to a substantially lower $T_{c}$ than others, especially in the BCS through unitary regimes. This is primarily because the $G G$ scheme double counts certain self energy diagrams. This theory is rather similar to the FLEX approximation of Scalapino and coworkers [119, 120] for the cuprates. Recently, Haussmann et al. [121] improved upon the NSR theory but found unphysical non-monotonic first-order-like behavior in the temperature dependence of entropy, $S(T)$.

It should be emphasized that none of these above mentioned theories contained pseudogap self energy in the $T_{c}$ equation. All NSR based theories essentially inherit the inconsistency of NSR treatment as well.

A pairing fluctuation theory which does contain a pseudogap was developed by the Levin group. Levin and coworkers [20-22] did intensive numerical study and found that a pseudogap opens up as $T$ approaches $T_{c}$ from above once the pairing correlation self energy is fed back into the $T_{c}$ equation. Chen, Kosztin and coworkers [23, 25] extended this work into a systematic theory for the superfluid state, and applied it to $d$-wave cuprate superconductors. With proper inclusion of low dimensionality and lattice effects [24], Chen et al.
[23] found an excellent (semi-quantitative) agreement of their computed cuprate phase diagram with that observed experimentally. This theory also gives a very natural explanation of the anomalous quasi-universal behavior of the superfluid density as a function of $T$ for different hole doping concentrations. In contrast to other theories mentioned above, pseudogap is a natural unavoidable consequence of strong pairing correlations in this theory.

Now with experimental evidence of a pseudogap in atomic Fermi gases, more people are finding in their theories evidence of a pseudogap [122-124]. Various quantum Monte Carlo simulations are also finding a pseudogap at unitarity above $T_{c}$.

\section{B. Pairing fluctuation theory for the pseudogap}

In this subsection, we will present a particular pairing fluctuation theory, in which the pairing correlation self energy is fed back into the $T_{c}$ and gap equation in a self-consistent fashion.

As in all pairing fluctuation theories, the key difference between this theory and the BCS mean field theory is that it includes finite center-of-mass momentum pairing. It is the finite momentum pairing that will give rise to a self energy beyond the strict BCS mean-field treatment.

What makes this theory unique is that finite momentum pairs and single particles are treated on an equal footing. As a consequence, these finite momentum pairs will cause a single particle excitation gap without phase coherence. In fact, the physical picture here is very intuitive. When strong pairing correlations are present, to excite a single fermion above $T_{c}$, one necessarily has to pay extra energy in order to break the pairing. This extra energy is associated with the pseudogap. In the BCS limit, this extra energy is negligible. However, in the BEC limit, stable two-body bound pairs will form at high $T$ so that one has to pay at least the binding energy to break the pairs. In the unitary or crossover regime, the pairs are meta-stable with a zero two-body binding energy so that the pseudogap is most pronounced. Needless to say, very much like the superfluid order parameter, the pseudogap is a many-body effect. While the pseudogap persists deep into the BEC regime, where a Fermi surface no longer exists, the big two-body binding energy may obscure the pseudogap effects. While the low energy excitations are Bogoliubov quasiparticles and finite momentum pairs for the BCS and BEC limits, respectively, a mix of both types necessarily takes place in the crossover regime. This is a requirement of the smoothness of the crossover.

The derivation of this theory [56] follows the early work of Kadanoff and Martin [125]. Through the equation of motion approach with a truncation of the infinite series of equations at the three-particle level, and decomposing the three particle Green's function $G_{3}$ into a sum of products of single particle Green's function $G$ and two particle Green's function $G_{2}$, we rigorously derived our self-consistent set of equations, with reasonable simplifications. While our equations may be conveniently cast diagrammatically into a $T$-matrix approxi- 


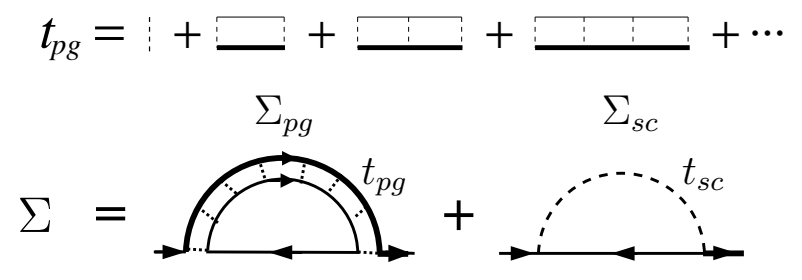

Figure 6. Feynman diagrams of the particle-particle scattering $T$ matrix $t_{p g}$ and the single particle self energy $\Sigma$. The self energy $\Sigma$ contains two contributions, from the condensate and finite momentum pairs, respectiveily.

mation, we emphasize that this theory is not a diagrammatic approach. For example, the pair susceptibility $\chi$ consists of a mix of bare Green's function $G_{0}$ and full Green's function $G$. This mix is not an ad hoc diagrammatic choice, but rather a natural consequence of the equation of motion approach. A main and nice feature of this theory is that it naturally recovers the BCS-Leggett result at zero $T$ and in the BCS limit. In addition, throughout the superfluid phase, our Green's function and the equations take the BCS form, except for the extra pseudogap contribution in the quasiparticle dispersion. Finally, the pseudogap (squared) is directly proportional to the density of finite momentum pairs, so that it provides a good measure of the contributions of finite momentum pairing fluctuations.

Instead of giving a full derivation of the theory, which can be found elsewhere [56], here we only give a summary and present the key equations so that we can focus on the physical picture. In addition, here we only consider the one-channel model, which is appropriate for high $T_{c}$ superconductors as well as atomic Fermi gases with a wide Feshbach resonance. A two-channel version of this theory can be found in Refs. [1, 126].

It is known that superfluidity concerns primarily the particle-particle channel. The main processes are summarized in the Feynman diagrams shown in Fig. 6. Here the finite momentum $T$-matrix $t_{p g}$ may be regarded as (the central part of) a two particle propagator, and the dashed line represents nonpropagating, zero-momentum pairs in the condensate. The self-energy $\Sigma$ of the single fermions comes from scattering with condensed and non-condensed pairs. Alternatively, a fermion may decay into a pair and a hole, which then recombine at a later point in spacetime, as shown in the second line in the figure. From the first line, it is not hard to conclude that the $T$-matrix can be regarded as a renormalized pairing interaction. Indeed, summing up the ladder diagrams, one obtain

$$
t_{p g}(Q)=\frac{U}{1+U \chi(Q)},
$$

with the same dimensionality as the interaction, where we have assumed a separable pairing interaction $V_{\mathbf{k}, \mathbf{k}^{\prime}}=$ $U \varphi_{\mathbf{k}} \varphi_{\mathbf{k}^{\prime}}$, with $\varphi_{\mathbf{k}}=1$ for a short range contact potential in atomic Fermi gases and $\varphi_{\mathbf{k}}=\cos k_{x}-\cos k_{y}$ for $d$-wave cuprate superconductors [127]. Here the pair susceptibility

$$
\chi(Q)=\sum_{K} G_{0}(Q-K) G(K) \varphi_{\mathbf{k}-\mathbf{q} / 2}^{2} .
$$

For clarity, a four-vector notation has been used, i.e., $K=$ $\left(i \omega_{l}, \mathbf{k}\right), Q=\left(i \Omega_{n}, \mathbf{q}\right), \sum_{K} \equiv T \sum_{l} \sum_{\mathbf{k}}$, etc., where $\Omega_{n}=$ $2 n \pi T$ and $\omega_{l}=(2 l+1) \pi T$ are even and odd Matsubara frequencies, respectively. Here and throughout we shall use the natural units $\hbar=k_{B}=1$ and set the volume to unity.

From Fig. 6, it is straightforward to write down the selfenergy $\Sigma$ and its superconducting component $\Sigma_{s c}$ and pseudogap component $\Sigma_{p g}$, as follows.

$$
\begin{aligned}
\Sigma(K) & =\Sigma_{s c}(K)+\Sigma_{p g}(K), \\
\Sigma_{s c}(K) & =-\Delta_{s c}^{2} G_{0}(-K) \varphi_{\mathbf{k}}^{2}=\frac{\Delta_{s c}^{2} \varphi_{\mathbf{k}}^{2}}{i \omega_{l}+\xi_{\mathbf{k}}}, \\
\Sigma_{p g}(K) & =\sum_{Q} t_{p g}(Q) G_{0}(Q-K) \varphi_{\mathbf{k}-\mathbf{q} / \mathbf{2}}^{2},
\end{aligned}
$$

where $\xi_{\mathbf{k}}=\epsilon_{\mathbf{k}}-\mu$ is the free fermion dispersion measured with respect to the Fermi level.

At this point, an approximation is needed in order to simplify the final result. Notice that pairing instability condition, i.e., the Thouless criterion, $t_{p g}^{-1}(0)=0$, implies that the main contribution in Eq. (4c) comes from the vicinity of $Q=0$. This leads to a good mathematical simplification over the complicated convolution,

$$
\Sigma_{p g}(K) \approx\left[\sum_{Q} t_{p g}(Q)\right] G_{0}(-K) \varphi_{\mathbf{k}}^{2}=-\Delta_{p g}^{2} G_{0}(-K) \varphi_{\mathbf{k}}^{2},
$$

where we have defined the pseudogap $\Delta_{p g}$ via

$$
\Delta_{p g}^{2}=-\sum_{Q} t_{p g}(Q)
$$

Now it is clear that, under approximation Eq. (5), we have the total self energy in the BCS form,

$$
\Sigma(K)=-\Delta^{2} G_{0}(-K) \varphi_{\mathbf{k}}^{2},
$$

where we have defined a total excitation gap $\Delta$ via

$$
\Delta^{2}=\Delta_{s c}^{2}+\Delta_{p g}^{2}
$$

Therefore, one immediately concludes that the full Green's function $G(K)$ also takes the BCS form,

$$
G(K)=\frac{u_{k}^{2}}{i \omega_{l}-E_{\mathbf{k}}}+\frac{v_{k}^{2}}{i \omega_{l}+E_{\mathbf{k}}},
$$

where $E_{\mathbf{k}}=\sqrt{\xi_{\mathbf{k}}^{2}+\Delta^{2} \varphi_{\mathbf{k}}^{2}}$ is the dispersion of the Bogoliubov quasiparticles, and $u_{\mathbf{k}}^{2}, v_{\mathbf{k}}^{2}=\frac{1}{2}\left(1 \pm \xi_{\mathbf{k}} / E_{\mathbf{k}}\right)$ are formally the usual BCS coherence factors.

Upon substituting the expressions for $G_{0}$ and $G$ into the Thouless criteria, $U^{-1}+\chi(0)=0$, one obtains immediately the gap (or $T_{c}$ ) equation after carrying out the Matsubara summation,

$$
1+U \sum_{\mathbf{k}} \frac{1-2 f\left(E_{\mathbf{k}}\right)}{2 E_{\mathbf{k}}} \varphi_{\mathbf{k}}^{2}=0
$$


where $f(x)$ is the Fermi distribution function. For a short range contact potential with $\varphi_{\mathbf{k}}=1$, as in atomic Fermi gases, one may conveniently regularize the ultraviolet divergence via the relation [128]

$$
\frac{m}{4 \pi a}=\frac{1}{U}+\sum_{\mathbf{k}} \frac{\varphi_{\mathbf{k}}^{2}}{2 \epsilon_{\mathbf{k}}}
$$

based on the Lippmann-Schwinger equation, so that the interaction strength $U$ is replaced with (the inverse of) the low energy $s$-wave scattering length $a$, which is a widely used experimental parameter in the AMO community. In this way, the gap equation becomes

$$
\frac{m}{4 \pi a}+\sum_{\mathbf{k}}\left[\frac{1-2 f\left(E_{\mathbf{k}}\right)}{2 E_{\mathbf{k}}}-\frac{1}{2 \epsilon_{\mathbf{k}}}\right] \varphi_{\mathbf{k}}^{2}=0 .
$$

Note that Eq. (11) defines a critical coupling $U_{c}$, which is more familiar to the condensed matter community; $U_{c}$ corresponds to the threshold for two fermions to form a bound state in vacuum, where the scattering length $a$ diverges,

$$
U_{c}=-1 / \sum_{\mathbf{k}} \frac{\varphi_{\mathbf{k}}^{2}}{2 \epsilon_{\mathbf{k}}} \text {. }
$$

Obviously, $U_{c}$ depends on the ultraviolet cutoff momentum in $\varphi_{\mathbf{k}}^{2}$. It is worth mentioning that in 2D and the contact limit in $3 \mathrm{D}, U_{c}$ goes to zero for an $s$-wave pairing interaction [129].

Now it should be emphasized that it is the mixed form of the pair susceptibility $\chi$ in Eq. (3) that gives rise to the BCS form in the gap equation (10). This is very satisfying since it is known that BCS theory works well in the weak coupling regime. Such a feature was already recognized in the early paper by Kadanoff and Martin [125]. Throughout the entire BCS-BEC crossover, this BCS form of gap equation reproduces the BCS-Leggett ground state [14]. This is an important merit of the present pairing fluctuation theory, since, while one may argue that the BCS-Leggett ground state is not perfect in the BEC regime, it has nonetheless been a basis for various theoretical works. It is apparent that the gap equation in other competing $T$-matrix approximations with a $G_{0} G_{0}$ or $G G$ in the pair susceptibility will deviate substantially from the BCS form.

Given the full Green's function Eq. (9), it is straightforward to write the fermion number constraint,

$$
n=2 \sum_{\mathbf{k}}\left[v_{k}^{2}+\frac{\xi_{\mathbf{k}}}{E_{\mathbf{k}}} f\left(E_{\mathbf{k}}\right)\right]
$$

which is the number equation.

Equations (10), (9) and (6) now forms a closed set of selfconsistent equations, which can be used to solve for $T_{c}$ and $\mu$, and $\Delta_{p g}$ at $T_{c}$, or for $\Delta, \mu$, and $\Delta_{p g}$ for $T<T_{c}$. To simplify and facilitate the computation of Eq. (6), we Taylor-expand the inverse $T$ matrix after analytic continuation, $i \Omega_{n} \rightarrow \Omega+$ $i 0^{+}$, as

$$
t_{p g}^{-1}(\Omega, \mathbf{q})=Z\left(\Omega-\frac{q^{2}}{2 M^{*}}+\mu_{\text {pair }}\right)
$$

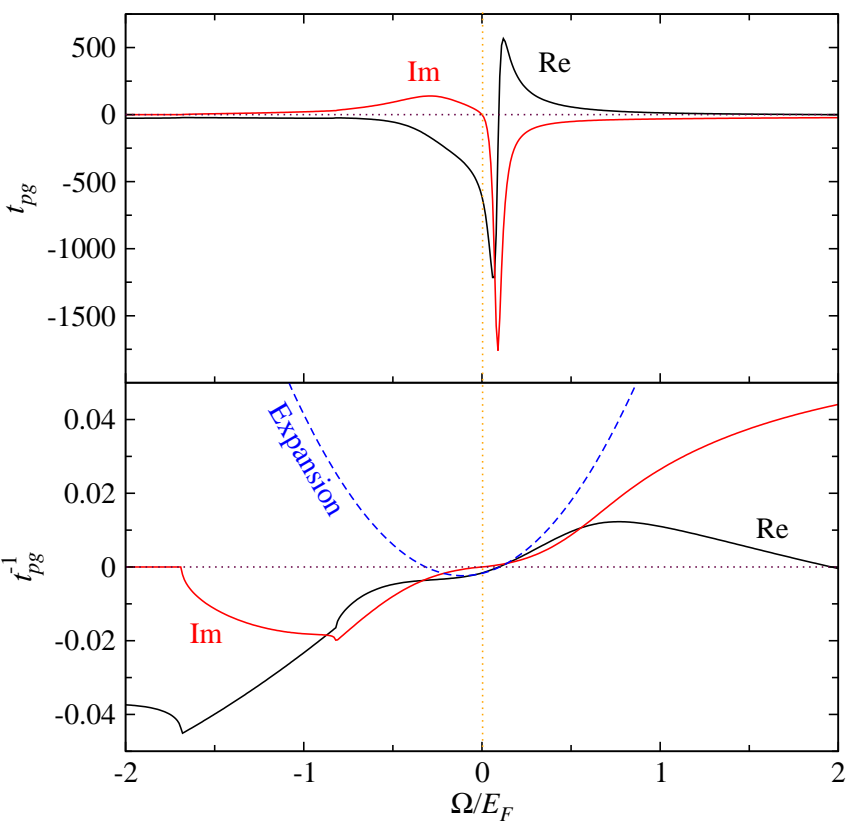

Figure 7. Typical behavior of the $T$-matrix and its inverse. Shown are calculated at unitarity $U=U_{c}$ at $T_{c}$, for a 3D continuum case with a finite range of interaction of the Lorentzian type $\varphi_{\mathbf{k}}^{2}=[1+$ $\left.\left(k / k_{0}\right)^{2}\right]^{-1}$, with $k_{0} / k_{F}=4$ and pair momentum $q / k_{F}=0.3$. Here Re and Im denote real and imaginary parts, respectively. The blue dashed curve is from the expansion of the inversion $T$-matrix, which coincides with the full $\operatorname{Re} t_{p g}^{-1}$ curve in the neighborhood of $\Omega=\Omega_{\mathbf{q}}$.

at the lowest order in $\Omega$ and q. A more elaborate treatment which includes higher order terms such as $\Omega^{2}$ as well as the imaginary part can be found in Ref. [56]. Use of such higher order expansion is made in cases where it makes a substantial (quantitative) difference [130, 131]. Here the inverse residue $Z$, the effective pair mass $M^{*}$, and the effective pair chemical potential $\mu_{\text {pair }}$ can be obtained in the process of the expansion. One can immediately extract the pair dispersion $\Omega_{\mathbf{q}}=q^{2} / 2 M^{*}-\mu_{\text {pair }}$. It now follows that

$$
Z \Delta_{p g}^{2} \approx \sum_{\mathbf{q}} b\left(\Omega_{\mathbf{q}}\right)
$$

where $b(x)$ is the usual Bose distribution function. Evidently, Eq. (16) suggests that $\Delta_{p g}^{2}$ represents the density of finite momentum pairs (up to a nearly constant coefficient).

Typical behaviors of the $T$-matrix $t_{p g}(\Omega, \mathbf{q})$ and its inverse are shown in Fig. 7. The curves are calculated for a 3D unitary Fermi gas at $T_{c}$, with a Lorentzian type of pairing potential, $\varphi_{\mathbf{k}}^{2}=\left[1+\left(k / k_{0}\right)^{2}\right]^{-1}$ at $k_{0} / k_{F}=4$ and $q / k_{F}=0.3$. From the lower panel, one can see that the Taylor expansion of the inverse $T$-matrix, $t_{p g}^{-1}(\Omega, \mathbf{q})$, up to the order of $\Omega^{2}$, agrees with the real part of the full curve very well near the dispersion relation $\Omega \approx \Omega_{\mathbf{q}}$, where the imaginary part, $\operatorname{Im} t_{p g}^{-1}(\Omega, \mathbf{q})$, becomes very small. This leads to a sharp resonance peak in $\operatorname{Im} t_{p g}(\Omega, \mathbf{q})$ at $\Omega \approx \Omega_{\mathbf{q}}$. This peak becomes sharper for smaller $q$ and at lower $T$, as expected. When the order parameter develops below $T_{c}$, for $q=0$, there is an extended range 


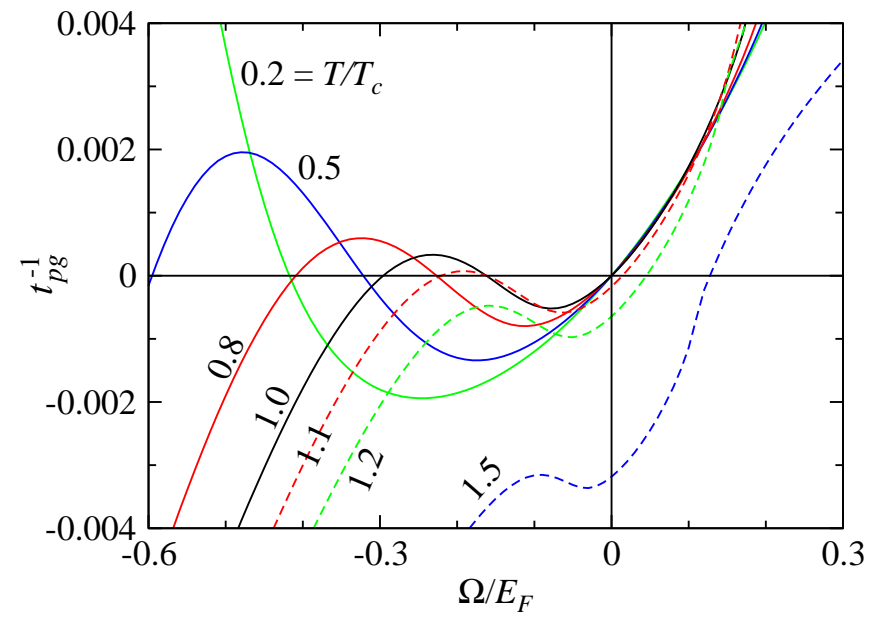

Figure 8. Typical behavior of the real part of the inverse $T$-matrix $t_{p g}^{-1}(\Omega, 0)$ near $\Omega=0$ for different $T$, as labeled, below (solid) and above $T_{c}$ (dashed curves). Shown are results calculated at unitarity $U=U_{c}$, for a $3 \mathrm{D}$ continuum case with a finite range of interaction of the Lorentzian type $\varphi_{\mathbf{k}}^{2}=\left[1+\left(k / k_{0}\right)^{2}\right]^{-1}$, with $k_{0} / k_{F}=4$.

of $\Omega$ at which $\operatorname{Im} t_{p g}^{-1}(\Omega, 0)$ vanishes. This is an effect of a finite excitation gap.

From the expansion Eq. (15), it is easy to see that the Thouless criterion requires

$$
\mu_{\text {pair }}=0, \quad \text { for } \quad T \leq T_{c},
$$

which is precisely the BEC condition of the (bosonic) fermion pairs. Therefore, it is transparent that the present pairing fluctuation theory unifies BCS theory and Bose-Einstein condensation using the BCS-BEC crossover picture; They are two sides of the same coin. Such a unification has not been made so obvious in other competing pairing fluctuation theories. Technically, it is the Taylor expansion Eq. (15) that has made this unification transparent; a similar expansion has not been seen in competing theories.

Indeed, as shown in Fig. 8, for different $T \leq T_{c}$ (solid curves), the real part $\operatorname{Re} t_{p g}^{-1}(\Omega, \mathbf{q}=\mathbf{0})$ always goes through the origin. However, for $T>T_{c}$ (dashed curves), this is no longer true. The nonzero intercept $t_{p g}^{-1}(0,0)=Z \mu_{\text {pair }}$ determines the effective pair chemical potential above $T_{c}$.

In fact, there are various situations where we need to know the approximate value of the pseudogap above $T_{c}$. In such a case, we need to extend the gap equation (10) or (12) to situations above $T_{c}$, as

$$
\frac{m}{4 \pi a}+\sum_{\mathbf{k}}\left[\frac{1-2 f\left(E_{\mathbf{k}}\right)}{2 E_{\mathbf{k}}}-\frac{1}{2 \epsilon_{\mathbf{k}}}\right] \varphi_{\mathbf{k}}^{2}=Z \mu_{\text {pair }} .
$$

The pseudogap equation is still given by Eq. (16) but with a nonzero $\mu_{\text {pair }}$, along with the number equation (14). Since $\Delta_{s c}=0$ above $T_{c}$, it is clear that the three unknowns are now $\left(\Delta_{p g}=\Delta, \mu, \mu_{\text {pair }}\right)$, as compared to $\left(\Delta_{p g}, \mu, \Delta_{s c}\right)$ below $T_{c}$.

It should be pointed out that as $T$ increases above $T_{c}$, the $T$ matrix $t_{p g}(Q)$ no longer diverges at $Q=0$. Therefore, Eq. (5) is no longer a good approximation for the pseudogap self energy $\Sigma_{p g}(K)$. In this sense, the use of the extended gap equation (18) should be restricted to a temperature regime not far above $T_{c}$, where $-\mu_{\text {pair }}$ is still very small.

Generalization of the above equations to population imbalanced as well as mass imbalanced situations is straightforward, which can be found in Refs. [130-135].

Finally, a few remarks are in order. The pseudogap self energy given in Eq. (4c) formally contains all contributions at the $T$-matrix level. However, by Eq. (5), the pseudogap self energy is approximated by a BCS-like, off-diagonal, coherent form. When the pseudogap $\Delta_{p g}$ vanishes, the pseudogap self energy is gone. Therefore, the diagonal incoherent contributions are dropped out. The incoherent contributions, $\delta \Sigma(K)$, is dominant in the weak coupling BCS limit, and becomes less important in the intermediate through strong coupling BEC regimes. It mainly causes a chemical potential shift, as well as a slight fermion mass renormalization. Such contributions are usually neglected in the study of superconductivity. Nevertheless, for atomic Fermi gases, as the strong couplings regime becomes accessible, it is known that these contributions have a substantial quantitative impact on the so-called beta factor at unitarity [136], which is defined as $1+\beta=\mu(0) / E_{F}$, where $\mu(0)$ and $E_{F}$ are the zero $T$ chemical potential at unitarity and the noninteracting Fermi energy, respectively. Without the incoherent contributions, the present theory produces the same prediction as the BCS meanfield result, $\beta \approx-0.41$, whereas the experimental values and quantum Monte Carlo (QMC) simulation results are found between -0.5 and $-0.7[49,137,138]$. When the incoherent contributions are included, theoretical calculations of Perali et al found $\beta \approx-0.545$, in better agreement with experiment, as expected. Here our attention is focused mainly on the moderate and strong coupling regimes, where the pseudogap effect is strong so that the incoherent self energy contribution is less important and only causes minor quantitative corrections.

Despite the simple BCS form of the self energy, Eq. (7), our result does include the contributions of pairing fluctuations, as in Eq. (4c). It is the simplification via Eq. (5) that encapsulates the fluctuations into a single parameter, $\Delta_{p g}$, via an integration of the fluctuation spectrum, as given in Eq. (6).

\section{Extended to Fermi gases in a trap}

When placed in a 3D isotropic harmonic trap, with a trapping potential $V(r)=\frac{1}{2} m \omega^{2} r^{2}$, one can resort to the local density approximation (LDA), by imposing a local chemical potential $\mu(r)=\mu-V(r)$ and the total particle number constraint,

$$
N=\int \mathrm{d}^{3} r n(r),
$$

where $n(r)$ is the local number density, and $\mu \equiv \mu_{0}=\mu(0)$ is the chemical potential at the trap center, often referred to as the global chemical potential. Note that here the trap potential does not necessarily have to be isotropic; it may be anisotropic with a variable aspect ratio, including the quasi-2D pancake or quasi-1D cigar shapes as the limit of a large aspect ratio. It 
may also be an optical lattice, which we shall not cover in this review.

With LDA, at any given location, the fermions are subject to pairing. Below $T_{c}$, there exists a superfluid core in the center of the trap. Outside the core, the fermions may or may not be paired, depending on their concrete radial position and the strength of the pairing interaction. When the pairing correlation is strong, one expects to find a pseudogap in the outskirt of the superfluid core. Inside the superfluid core, the fermions locally satisfy the gap equation as well as the pseudogap equation, while the local chemical potential $\mu(r)$ determines the local density $n(r)$. Outside the superfluid core, the fermions are in the normal state, so that the effective pair chemical potential $\mu_{\text {pair }}$ becomes nonzero. In this case, we need to use the extended gap equation (18) in place of equation (10). As mentioned earlier, the use of the extended gap equation (18) should be restricted to a temperature regime not far above $T_{c}$. In the trapped case, this translates into a narrow shell outside the superfluid core. Nevertheless, as the density gets lower towards the trap edge, the gap becomes small and the error introduced into the total number $N$ via the local $n(r)$ is negligible. Thus in our actual numerical calculations, we apply Eq. (18) for the entire shell of Fermi gases outside the superfluid core, and switch to unpaired normal Fermi gas state when the gap becomes tiny, e.g., when $\Delta<10^{-5}$.

\section{Thermodynamics and superfluid density}

The pseudogap and finite momentum pair excitations necessarily affect the thermodynamic behavior and transport properties, such as the superfluid density. Away from the BCS regime, both Bogoliubov quasiparticles and finite momentum pairs are present at finite $T$. They serve to destroy the superfluid density and contribute to the entropy. Knowing the excitation spectra, it is straightforward to write down the entropy $S$, as a sum of fermionic $\left(S_{f}\right)$ and bosonic $\left(S_{b}\right)$ contributions. In a trap, the total entropy involves an integral over the trap, given by $S=\int \mathrm{d}^{3} r s(r)$ (and similarly for $S_{f}$ and $S_{b}$ ), where

$$
\begin{aligned}
s & =s_{f}+s_{b}, \\
s_{f} & =-2 \sum_{\mathbf{k}}\left[f_{k} \ln f_{k}+\left(1-f_{k}\right) \ln \left(1-f_{k}\right)\right], \\
s_{b} & =-\sum_{q \neq 0}\left[b_{q} \ln b_{q}-\left(1+b_{q}\right) \ln \left(1+b_{q}\right)\right] .
\end{aligned}
$$

Here $f_{k} \equiv f\left(E_{\mathbf{k}}\right)$, and $b_{q} \equiv b\left(\Omega_{\mathbf{q}}-\mu_{\text {pair }}\right)$. The fermion contribution coincides formally with the standard BCS result for noninteracting Bogoliubov quasiparticles [although here $\left.\Delta\left(T_{c}\right) \neq 0\right]$. And the bosonic contribution is given by the expression for non-directly-interacting bosonic pairs with dispersion $\Omega_{\mathbf{q}}$, with an effective mass $M^{*}$ which is not necessarily equal to $2 \mathrm{~m}$. When the chemical potential $\mu$ becomes negative, the entropy $S$ becomes dominantly bosonic, since the fermionic part $S_{f}$ becomes exponentially suppressed.

One can also write down the energy of the Fermi gas, which consists of a fermionic and bosonic part in a similar fashion.
Thus, in a trap, the local energy is given by

$$
\begin{aligned}
E & =\mu n(r)+E_{f}+E_{b}, \\
E_{f} & =\sum_{K}\left(i \omega_{n}+\epsilon_{\mathbf{k}}-\mu(r)\right) G(K) \\
& =\sum_{\mathbf{k}}\left[2 E_{\mathbf{k}} f_{k}-\left(E_{\mathbf{k}}-\epsilon_{\mathbf{k}}+\mu(r)\right)\right]+\Delta^{2} \chi(0), \\
E_{b} & =\sum_{q}\left(\Omega_{\mathbf{q}}-\mu_{\text {pair }}\right) b_{q},
\end{aligned}
$$

where the pair susceptibility $\chi(0)$ is given by Eq. (3) at $Q=0$. One obtains the total energy by integrating Eqs. (21) over the entire trap.

To end this subsection, we present the expression for the superfluid density, which can be derived using the linear response theory with a generalized Ward identity [25, 56, 139]. In a homogeneous case, it is given by

$$
\begin{aligned}
\left(\frac{n_{s}}{m}\right)= & \frac{2}{d} \sum_{\mathbf{k}} \frac{\Delta_{s c}^{2}}{E_{\mathbf{k}}^{2}}\left[\frac{1-2 f\left(E_{\mathbf{k}}\right)}{2 E_{\mathbf{k}}}+f^{\prime}\left(E_{\mathbf{k}}\right)\right] \\
& \times\left[\left(\vec{\nabla}_{\mathbf{k}} \xi_{\mathbf{k}}\right)^{2} \varphi_{\mathbf{k}}^{2}-\frac{1}{4}\left(\vec{\nabla}_{\mathbf{k}} \xi_{\mathbf{k}}^{2}\right) \cdot\left(\vec{\nabla}_{\mathbf{k}} \varphi_{\mathbf{k}}^{2}\right)\right] \\
= & \frac{\Delta_{s c}^{2}}{\Delta^{2}}\left(\frac{n_{s}}{m}\right)^{M F}
\end{aligned}
$$

where $d=3$ is the dimensionality, $f^{\prime}(x)=\mathrm{d} f(x) / \mathrm{d} x$, and we have kept $\left(n_{s} / m\right)$ as a combination since on a lattice the mass $m$ is not well defined, but the combination is. The key result here is the last line in Eq. (22), where $\left(n_{s} / m\right)^{M F}$ is the BCS mean-field expression for $\left(n_{s} / m\right)$, which necessarily persists into the normal state when a pseudogap exists above $T_{c}$. It is the prefactor $\Delta_{s c}^{2} / \Delta^{2}$ that guarantees that there be no Meissner effects above $T_{c}$. Indeed, within the present pairing fluctuation theory, the superfluid density vanishes continuously and nicely as $T$ approaches $T_{c}$ from below, following the $T$ dependence of $\Delta_{s c}^{2}$ in the vicinity of $T_{c}$. A population imbalanced version of Eq. (22) can be found in Ref. [140]. In a trap, all one needs to do is to integrate the local superfluid density $n_{s}(r)$ over the entire trap, $N_{s}=\int \mathrm{d}^{3} r n_{s}(r)$.

\section{KEY RESULTS OF THE PRESENT PAIRING FLUCTUATION THEORY}

In this section, we will present some key results related to the pseudogap phenomena. We first present the results for a dilute two-component 3D Fermi gas with a short range $s$-wave pairing interaction, which serves to demonstrate the simple physical picture, and will be a basis of comparison for other cases. Next we shall present the main results for the cuprate superconductors, and then quickly switch to results relevant for atomic Fermi gases, which is the main subject of this Review. 

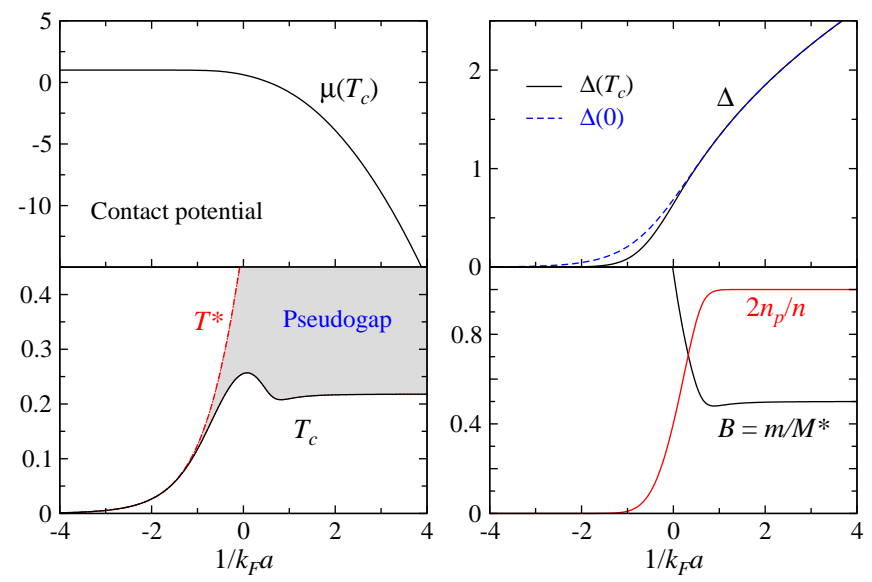

Figure 9. Calculated phase diagram and $T_{c}, T^{*}, \mu\left(T_{c}\right), \Delta(0), \Delta\left(T_{c}\right)$ and pair fraction $2 n_{p} / n$ and inverse pair mass $m / M^{*}$ (at $T_{c}$ ) of a $3 \mathrm{D}$ homogeneous Fermi gas with a contact potential as a function of $1 / k_{F} a$. Here $n_{p}$ denotes the number density of pairs.

\section{A. Two-component homogeneous Fermi gases in the 3D continuum}

Figure 9 summarizes the main results of the present theory on the behavior of a 3D Fermi gas with a contact potential. Shown are the phase diagram and related quantities, including $T_{c}, T^{*}, \mu\left(T_{c}\right), \Delta(0), \Delta\left(T_{c}\right)$, as well as the pair fraction $2 n_{p} / n$ and the effective inverse pair mass $m / M^{*}$ at $T_{c}$. Here the pair formation temperature $T^{*}$, as a crossover temperature, is approximated by the mean field solution of $T_{c}$. While the $T_{c}$ curve is close to its mean-field counterpart in the weak coupling BCS regime, a (shaded) pseudogap phase emerges in the intermediate (crossover or unitary) through strong pairing BEC regimes. Along with the BCS-BEC crossover, the fermionic chemical potential $\mu$ decreases from $E_{F}$ in the noninteracting limit, and approaches a large negative given by $-E_{b} / 2$ in the deep BEC regime, where $E_{b}$ is the two-body binding energy. At the same time, the excitation gaps $\Delta(0)$ and $\Delta\left(T_{c}\right)$, at zero $T$ and $T_{c}$, respectively, grow with $1 / k_{F} a$. While $\Delta\left(T_{c}\right)$ at $T_{c}$ roughly vanishes in the BCS regime, it becomes nearly equal to $\Delta(0)$ in the BEC regime. A scrutiny also reveals that the ratio $\Delta /|\mu|$ approaches 0 in the BEC limit, implying that in the deep BEC regime, many-body effects are relatively unimportant so that pairing is dominated by two-body physics. Indeed, the curve of $2 n_{p} / n$ shows that in the BEC regime, essentially all fermions form pairs. A calculation of the pair size reveals that it shrinks in real space with increasing pairing strength $[1,56]$, leading to a dilute Bose gas of tightly bound fermion pairs in the deep BEC regime.

One feature that seems unique to the present theory is that the $T_{c}$ curve reaches a maximum near unitarity. At the same time, there is a minimum where $\mu$ changes sign. In the BEC limit, $T_{c}$ approaches its ideal BEC asymptote from below, as expected on physical grounds. This nonmonotonic behavior of $T_{c}$ can be readily explained. Starting from the intermediate pairing strength regime, the formation of pairs quickly depletes the effective density of fermions, making the effective

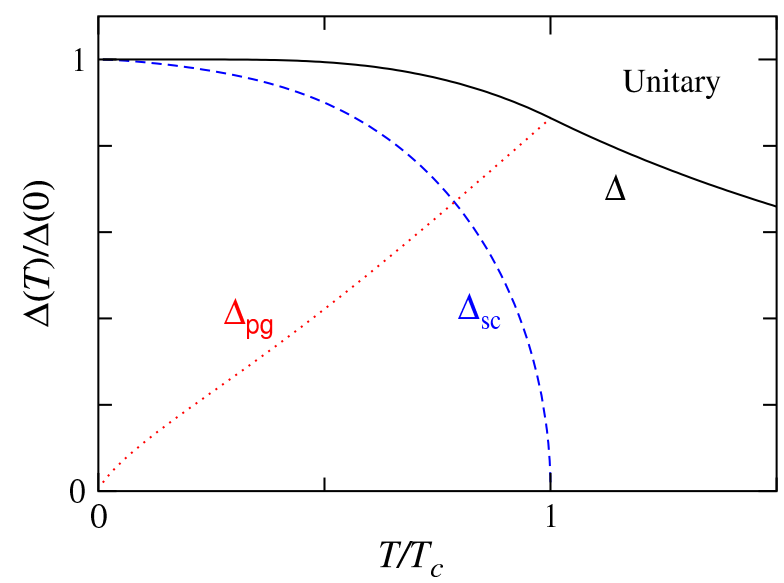

Figure 10. Normalized gaps as a function of reduced temperature $T / T_{c}$ at unitarity. The gap at $T_{c}$ is comparable to the gap at $T=0$. The curves are calculated for a homogeneous 3D Fermi gas in continuum, with pairing symmetry $\varphi_{\mathbf{k}}^{2}=1 /\left[1+\left(k / k_{0}\right)^{2}\right]$ at $k_{0} / k_{F}=4$.

fermionic density of state decrease, associated with a shrinking Fermi surface. This leads to a decrease in $T_{c}$. On the other hand, the bosonic part of the system emerges and grows, as given by the increasing pair density $n_{p}$. Beyond the $\mu=0$ point, the Fermi surface completely vanishes, and $n_{p}$ reaches its maximum value $n / 2$, so that $T_{c}$ is controlled by the BEC temperature, which increases slowly with $m / M^{*}$.

It should be emphasized that, as shown by the $m / M^{*}$ curve, except in the deep BEC regime, the effective pair mass differ significantly from $2 m$. This should be contrasted with NSRbased theories, which has $M^{*}=2 m$ in all cases.

As one can see from Fig. 9, in the pseudogap phase, the pseudogap $\Delta\left(T_{c}\right)$ and the pair density $n_{p}$ grow hand in hand.

Figure 10 illustrates the behavior of the gaps as a function of temperature for a 3D homogeneous Fermi gas at unitarity. The pseudogap at $T_{c}$ is close to the zero $T$ gap $\Delta(0)$. For weaker coupling toward the BCS limit, the pseudogap $\Delta_{p g} / \Delta(0)$ decreases and vanishes eventually. On the contrary, with increasing pairing strength toward the $\mathrm{BEC}$ regime, the ratio $\Delta\left(T_{c}\right) / \Delta(0)$ approaches unity so that the gap becomes essentially temperature independent except at very high $T$. At low $T$, following Eq. (16), the pseudogap scales as $\Delta_{p g}(T) \propto T^{3 / 4}$.

In Fig. 11, we present the typical density of states $N(\omega)=$ $-2 \sum_{\mathbf{k}} \operatorname{Im} G(\omega+i 0, \mathbf{k})$ for (a) BEC, (b) BCS and (c) pseudogap regimes for a homogeneous $3 \mathrm{D}$ Fermi gas, at different temperatures from slightly above $T_{c}$ down to $0.5 T_{c}$, half way into the superfluid phase. Note that to distinguish the incoherent pair contributions from that of the condensate to the self energy, we have used a more realistic form of the pseudogap self energy,

$$
\Sigma_{p g}(\omega, \mathbf{k}) \approx \frac{\Delta_{p g}^{2}}{\omega+\xi_{\mathbf{k}}+i \gamma},
$$

where $\gamma$ is treated as a phenomenological parameter independent of temperature [141]. The very low but finite DOS for $\omega<0$ in the BEC regime is purely a consequence of particle- 


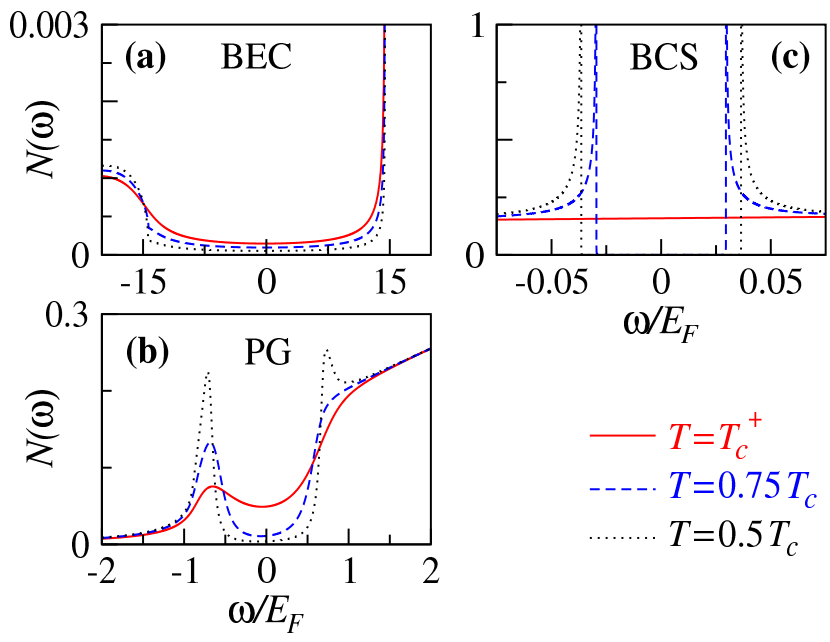

Figure 11. Fermionic density of states vs energy for the three regimes at three indicated temperatures from slightly above $T_{c}$ down to the superfluid phase. Note the big difference in the scales. Replotted from Ref. [126].

hole mixing due to pairing. Except for the BCS regime, where the gap closes at $T_{c}$, a pseudogap is already present at $T_{c}$ for both the BEC and the pseudogap regimes.

From Fig. 11, one can easily conclude that unless one has a very high resolution in experiment, one can no longer use the opening of a gap in the DOS as a signature of superfluid transition in the presence of a pseudogap. Instead, it is a signature of pairing which in general takes place before superfluid phase coherence sets in. This is a very important effect of the pseudogap.

Shown in Fig. 12 is the normalized superfluid density $n_{s} / n$ in a 3D homogeneous Fermi gas as a function of the reduced temperature $T / T_{c}$, for different pairing strengths $U / U_{c}=0.7$, 1.0 , and 1.5 , corresponding to the BCS, unitary, and BEC regimes, respectively. In comparison with the exponential $T$ dependence of the BCS case (black solid curve), a clear deviation can be seen in the unitary case (blue dashed curve) already. This is due to the bosonic pair excitations, which obey the $T^{3 / 2}$ power law at low $T$. In the BEC regime (red dot-dashed curve), the low $T$ behavior is dominated by the $T^{3 / 2}$ power law of the bosonic excitations. Indeed, in the BEC regime, fermionic quasiparticles are essentially absent below $T_{c}$, due to the large negative chemical potential $\mu$.

Figure 12 confirms that in our theory, due to the generalized Ward identity [25], Meissner effect is necessarily absent above $T_{c}$. In this way, our superfluid density vanishes nicely at $T_{c}$, unlike some competing scenarios (see, e.g., theories based on NSR) which predicts a first order jump or nonmonotonic temperature dependence at $T_{c}[117,121,142,143]$.

\section{B. Application for the cuprates: quasi-2D superconductors on a lattice with a $d$-wave pairing symmetry}

When the pairing fluctuation theory is applied to a quasi-2D lattice, it turns out that the lattice periodicity and the low di-

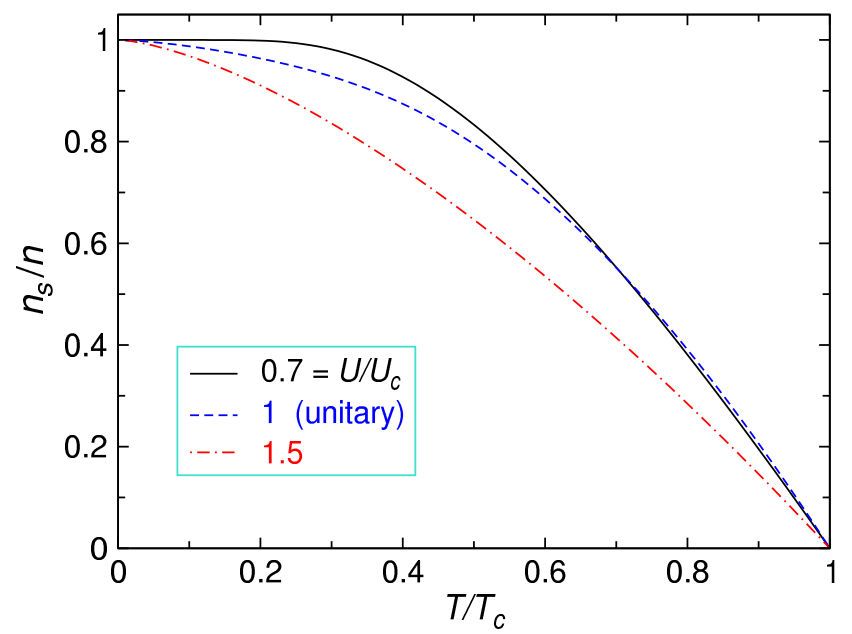

Figure 12. Normalized superfluid density $n_{s} / n$ of a 3D homogeneous Fermi gas as a function of $T / T_{c}$ for three different regimes. Here $U / U_{c}=1$ is equivalent to $1 / k_{F} a=0$, the unitary limit. The pseudogap or finite momentum pairs contribute a $T^{3 / 2}$ power law to the low $T$ dependence, which becomes dominant as $U / U_{c}$ increases. The calculation was done for an NSR type of potential, $\varphi_{\mathbf{k}}^{2}=1 /\left[1+\left(k / k_{0}\right)^{2}\right]$ with $k_{0} / k_{F}=4$.

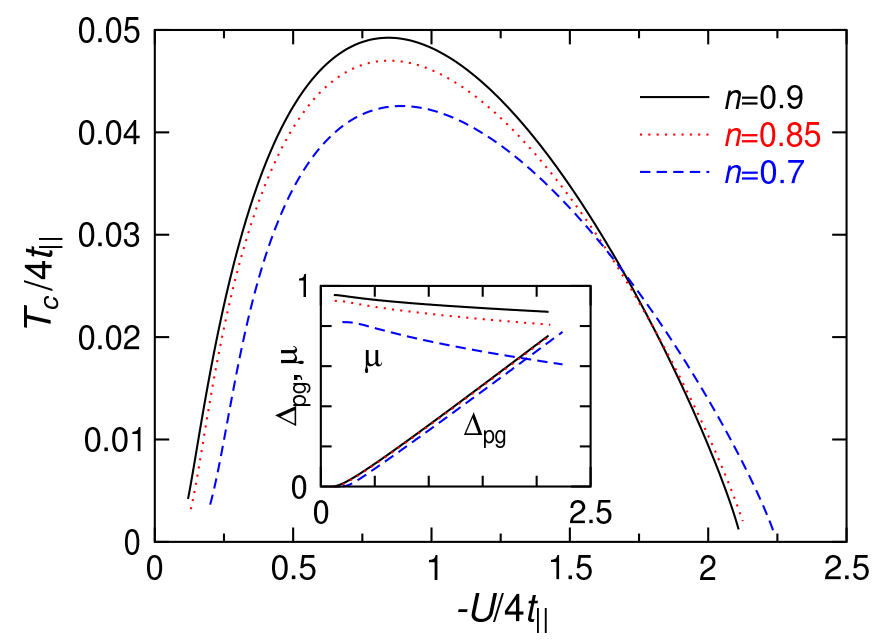

Figure 13. Superfluid transition temperature $T_{c}$ as a function of $-U / 4 t_{\|}$for fermions on a quasi-2D square lattice, with a $d$-wave pairing symmetry, at density $n=0.9$ (black solid line), 0.85 (red dotted), and 0.7 (blue dashed line). Shown in the inset are corresponding $\Delta\left(T_{c}\right)$ and $\mu$. The system is deep in the fermionic regime when $T_{c}$ vanishes, where the chemical potential $\mu$ is not far from its noninteracting value. Here $t_{\perp} / t_{\|}=0.01$. Taken from Ref. [24].

mensionality bear important consequences. The periodic lattice imposes an upper cut-off in the momentum space, and fermion pairs have to move via virtual ionization. As a result, the superfluid transition temperature $T_{c}$ scales as $t_{\|}^{2} / U$ at low densities, where $t_{\|}$is the in-plane nearest neighbor hopping integral, and $U$ is the on-site attractive (pairing) interaction. At high densities, calculations show that $T_{c}$ vanishes abruptly at an intermediate pairing strength so that the BEC regime is not accessible. For high $T_{c}$ superconductors, the $d$-wave pair- 

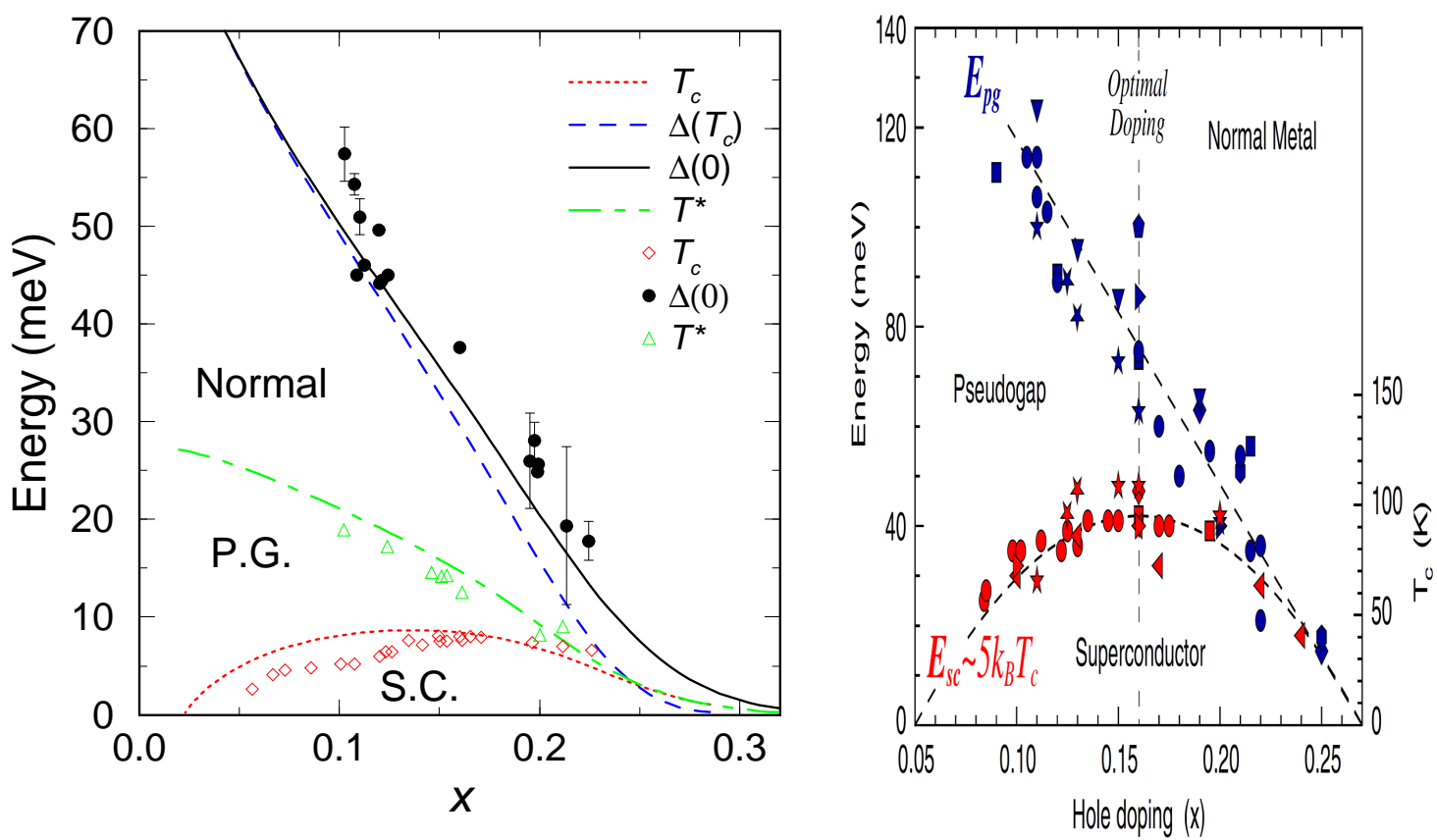

Figure 14. (Left) Cuprate phase diagram, taken from Ref. [56], showing $\Delta(0), T_{c}, \Delta\left(T_{c}\right)$, and $T^{*}$, calculated for $-U / 4 t_{0}=0.047$, and $t_{\perp} / t_{\|}=0.003$. Shown as symbols are experimental data. The normal, pseudogap, and superconducting phases are labeled with "Normal", "P.G.", and "S.C.", respectively. (Right) Plot of a recent collection of experimentally measured pseudogap data (with $E_{p g}=2 \Delta_{p g}$, blue symbols), taken from Ref. [145]. The right axis shows the temperature scales (for $T_{c}$ and $T^{*}$ ). Note that the right panel has been horizontally squeezed so that it can be overlaid on top of the left panel in the range of $x=0.05 \sim 0.27$. These experimental pseudogap data in the right panel agree with the blue dashed curve for $\Delta_{p g}=\Delta\left(T_{c}\right)$ in the left panel very well.

ing symmetry further restricts the lower bound of the pair size to that of a unit cell. Along with the non-local effect [144] of the $d$-wave pairing, the system is essentially always in the high density regime so that $T_{c}$ vanishes abruptly at an intermediate pairing strength. The high density strongly suppresses the motion of the (finite size) pairs so that at certain point, the pairing strength is so strong that the pairs become localized, with a diverging effective mass $M^{*} \rightarrow \infty$. More details regarding the lattice, low dimensionality and $d$-wave effects may be found in Ref. [24].

Shown in Fig. 13 are the $T_{c}$ curves for a $d$-wave superconductor on a quasi-2D square lattice at relatively high densities relevant to the cuprate superconductors. For all three densities, $T_{c} / 4 t_{\|}$shuts off around 2.2. The chemical potential shown in the inset reveals that the system is still deep in the fermionic regime when $T_{c}$ vanishes abruptly. The $\Delta_{p g}$ curves show that the pseudogap effect is strong. More details regarding the lattice, low dimensionality and $d$-wave effects may be found in Ref. [24].

In Fig. 14, we present the theoretical cuprate phase diagram calculated using this theory (lines) and compare with experimental data (symbols) in the left panel. In our calculations, we take $U$ to be doping independent, and incorporate the effect of the Mott transition at half filling, by introducing a doping concentration $x$ dependence into the inplane hopping matrix elements $t_{\|}=t_{0} x$, as would be expected in the limit of strong on-site Coulomb interactions in a Hubbard model [146]. Therefore, except the weak logarith- mic dependence [24] of $T_{c}$ on the anisotropy $t_{\perp} / t_{\|}$, there is only one free parameter, i.e., $U / t_{0}$. Without further tweaking details such as next nearest neighbor hopping $t^{\prime}$, the agreement between theory and experiment in terms of low $T$ gap $\Delta(0), T^{*}$ and $T_{c}$ is remarkable. A later collection of pseudogap at and above $T_{c}$ are shown in the right panel, which is so scaled that a direct comparison can be made by overlaying it on top of the left panel. Note that the experimental $T_{c}$ data points in both panels fit the same empirical formula $T_{c}=T_{c}^{\max }\left[1-82.6(0.16-x)^{2}\right]$ fairly well $[145,147]$.

This remarkable (semi-)quantitative agreement between theory and experiment really distinguishes the present theory from other rival theories of high $T_{c}$ superconductivity. Despite the fact many different high $T_{c}$ theories have been proposed, one finds it awkward that it is hard to find a high $T_{c}$ theory that is capable of quantitative computations.

\section{3D Fermi gas in an isotropic trap}

When the typical pair size or coherence length is far smaller than the trap size (more precisely, the size of the Fermi gas cloud), LDA is a good approximation. In Fig. 15, we present the solution of $T_{c}$ under LDA as a function of $1 / k_{F}$ a for a Fermi gas in a 3D isotropic harmonic trap with a contact potential. With no surprise, a pseudogap is found to emerge as the pairing strength grows. The behavior of the pseudogap at $T_{c}$ is shown in the inset. Near unitarity, the plateau in the $T_{c}$ 


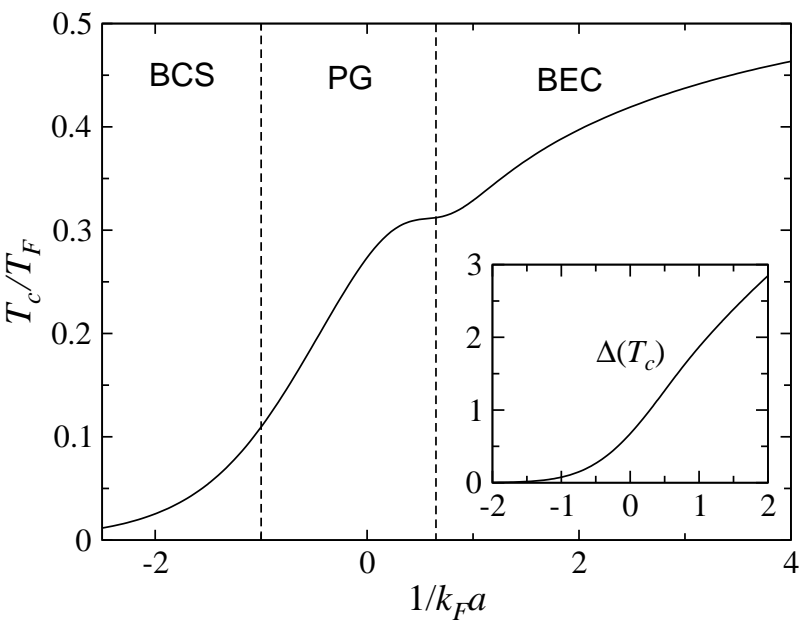

Figure 15. Behavior of $T_{c}$ as a function of $1 / k_{F} a$ for a Fermi gas in a $3 \mathrm{D}$ isotropic harmonic trap with a short range potential. The plateau is clearly a residue of the maximum-minimum feature in the $T_{c}$ curve of the homogeneous Fermi gas. As $1 / k_{F} a \rightarrow+\infty, T_{c}$ approaches its BEC asymptote in a trap, $0.518 T_{F}$, where $T_{F}$ is the global Fermi temperature in the noninteracting limit. The inset shows the behavior of corresponding $\Delta\left(T_{c}\right)$. Here "PG" denotes pseudogap.

curve is clearly a residue of the maximum-minimum feature in the $T_{c}$ curve of the homogeneous Fermi gas. Meanwhile, due to the shrunk cloud size and thus increased density at the trap center in the BEC regime, $T_{c}$ approaches a much greater BEC asymptote in a trap, $0.518 T_{F}$, as $1 / k_{F} a \rightarrow+\infty$, as compared to its homogeneous counterpart, $0.218 T_{F}$. Note that in a trap the global $T_{F}$ is defined by the Fermi temperature in the noninteracting limit. While the Fermi gas locally satisfies the gas equation as if it were in a homogeneous case, it is easy to conclude that from the weak coupling BCS limit through the deep BEC limit, the central density $n(0)$ is enhanced by a factor of $(0.518 / 0.218)^{3 / 2}=3.66$ by the pairing interaction. (Here we have made use of the relation $E_{F} \propto n^{2 / 3}$ for a homogeneous Fermi gas).

Shown in Fig. 16 in the evolution of the spatial density and gap profiles in the 3D harmonic trap as a function of the pairing strength. For illustration purpose, it suffices to focus in the near-BCS through near BEC regimes, without going to the extreme BCS or BEC limits. Indeed, it is the crossover or unitary regime, where the scattering length becomes large, that has been the focus of most studies. In the weak coupling limit, the scattering length is proportional to the interaction strength. For this reason, the unitary regime has often been referred to (mainly by the AMO community) as "strongly interacting". As can be seen from the figure, as the pairing strength increases, the Fermi gas cloud shrinks toward the trap center (upper panel), where the density necessarily increases as a result. At the same time, the spatial distribution of the pairing gap (lower panel) also becomes more focused at the trap center, despite its growing with the pairing strength.

To distinguish the present theory from competing theories, it is worth mentioning that the density profiles are smooth spatially, and evolve monotonically with temperature. This

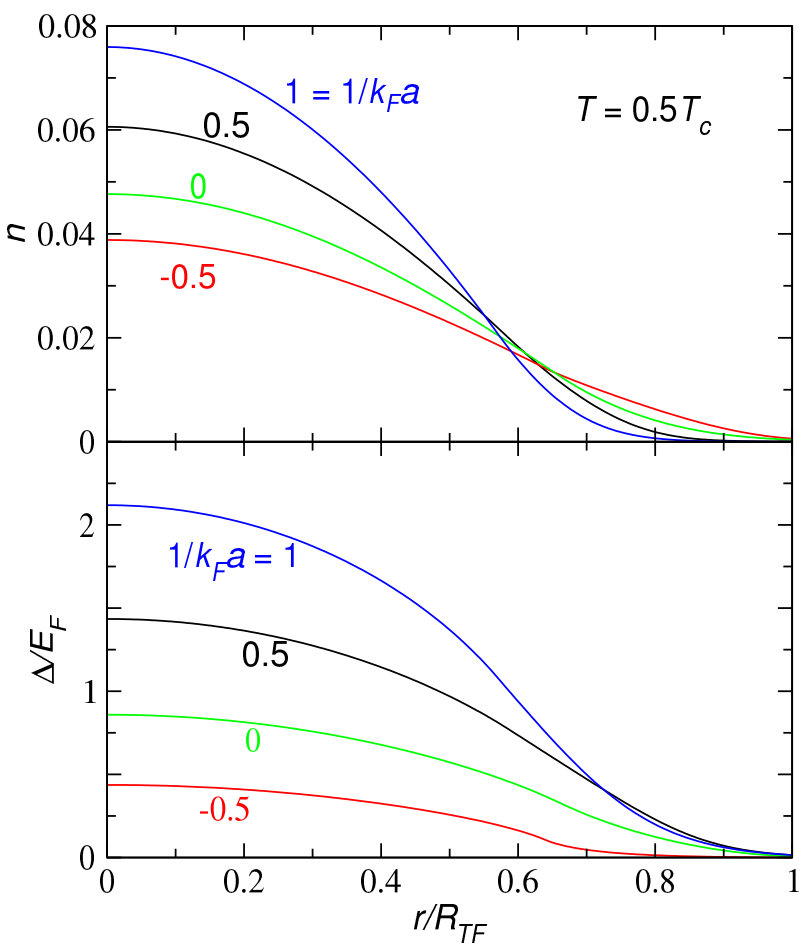

Figure 16. (a) Density and (b) gap profile of a Fermi gas in a 3D harmonic trap for various pairing strengths from (near-)BCS to BEC, as labeled. All curves are calculated half way below their corresponding $T_{c}$. Here $R_{T F}$ is the Thomas-Fermi (TF) radius, given by the zero $T$ radius in the noninteracting limit.

should be contrasted with the theory of Strinati and coworkers [116], which predicts non-monotonic radial dependence and non-monotonic temperature dependence.

It should also mentioned that mean-field calculations predict as a signature of superfluidity a kink at the edge of the superfluid core in the density profile [148, 149]. Such a kink is absent in our theory as well as experimental observations. Indeed, it can be easily shown that [56]

$$
\begin{aligned}
n & =2 Z \Delta^{2}+2 \sum_{\mathbf{k}} f\left(\xi_{\mathbf{k}}\right)=2 Z \Delta_{s c}^{2}+2 Z \Delta_{p g}^{2}+2 \sum_{\mathbf{k}} f\left(\xi_{\mathbf{k}}\right) \\
& \equiv 2 n_{c}+2 n_{p}+n_{f},
\end{aligned}
$$

where $n_{c} \equiv Z \Delta_{s c}^{2}$ is the number density of condensed Cooper pairs [150], $n_{f} \equiv 2 \sum_{\mathbf{k}} f\left(\epsilon_{\mathbf{k}}-\mu(r)\right)$ is the density of fermions as though they were free. In Fig. 17, we show the density profile $n(r)$ (black curve) and its component contributions from the condensate $2 n_{c}$ (green), finite momentum pairs $2 n_{p}$ (red) and free fermions $n_{f}$ (blue), for three representative temperatures $T / T_{c}=1,0.75$, and 0 . The right columns show the (de-)composition of the density. At $T / T_{c}=0.75$, the density profile is composed of all three components. It is evident that the contribution of finite momentum pairs (red area) is essential in eliminating the kink, which would exist otherwise at the edge of the superfluid core (green area). It is worth mentioning that finite momentum pair density $n_{p}$ (red curve) is nearly flat inside the superfluid core. This is because $\mu_{\text {pair }}=0$ and the effective pair mass is nearly the same 


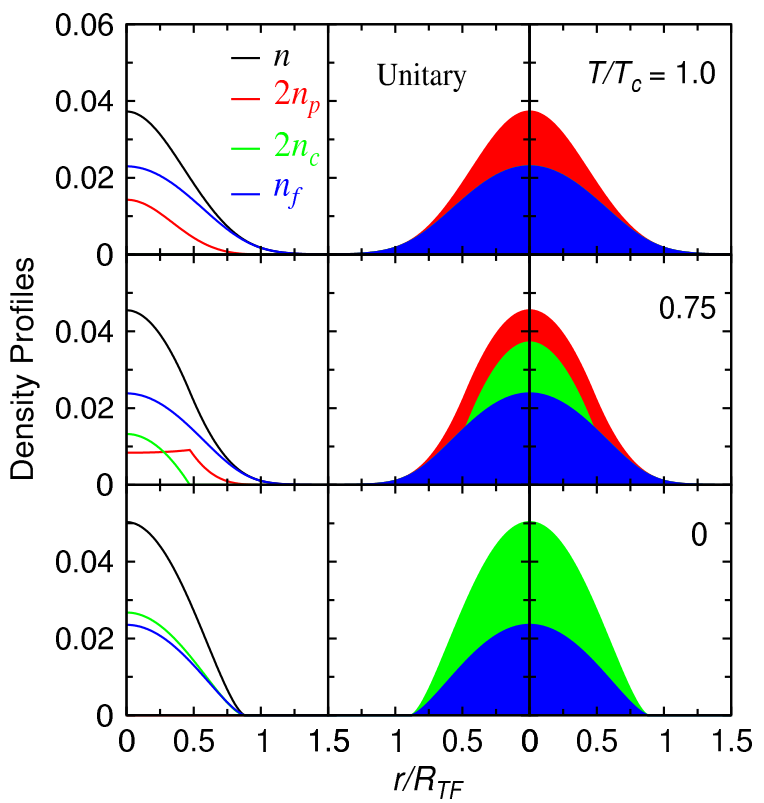

Figure 17. Decomposition of the density profile $n(r)$ of a Fermi gas in a trap at unitarity for representative temperatures $T / T_{c}=1,0.75$, and 0 , as labeled. At $T_{c}$, there are only finite momentum pairs (red) and fermions (blue). Below $T_{c}$, the condensate (green) develops, and the finite momentum pair contributions decreases. The pair density $n_{p}$ is nearly uniform inside the superfluid core. At $T=0$, finite momentum pairs disappear and all pairs are condensed.

across the core. Then $n_{p}$ starts to decrease gradually outside the core, when $-\mu_{\text {pair }}$ acquires a finite value and grows with radius. At $T_{c}$, the superfluid core disappears. On the other hand, at $T=0$, the finite momentum pairs disappear; all pairs are condensed. In the BCS mean-field theory, where $\Delta_{p g}=0$ and the fermion propagator contains no self energy feedback, Eq. (24) reduces to $n=2 n_{c}+n_{f}$. In fact, this is how the density was decomposed in Refs. [148, 149]. Figure 17 shows that without finite momentum pairs, there would be an unphysical kink at the edge of the superfluid core.

Interestingly, it is worth mentioning that under this decomposition, the condensate fraction (green area) $2 n_{c} / n$ is not $100 \%$ at $T=0$ at unitarity since $\mu>0$, even if the superfluid density is. This result is shown in Fig. 18, where the condensate fraction is plotted as a function of pairing strength for the entire BCS-BEC crossover for a short range potential in both the trapped (black solid) and homogeneous (red dashed line) cases. As one can imagine from Eq. (24), the figure shows that the condensate fraction does not rise to $100 \%$ until the $\mathrm{BEC}$ regime is reached, where $\mu$ changes sign and becomes negative. To understand the small condensate fraction in the BCS regime, one notices that, based on Eq. (24), at zero $T$ this fraction covers the rest part that is not accounted for by the $n_{f}$ term. Therefore, it is a measure of the extent to which a fermion lives a life as a component of a Cooper pair rather than an individual fermion. At unitarity, the condensate fraction is about 0.55 in the homogeneous case, and 0.53 in the trap, respectively. These numbers are close to that from quantum Monte Carlo simulations [151], 0.57 for a total particle

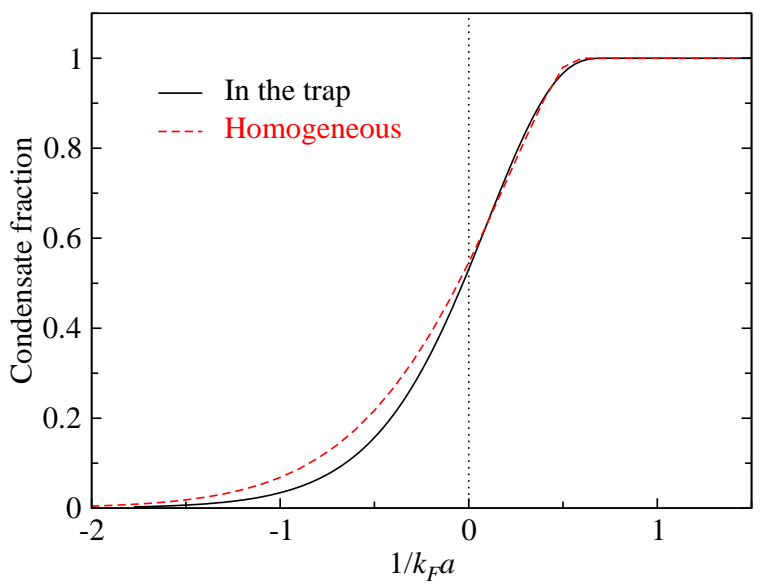

Figure 18. Condensate fraction at $T=0$ as a function of $1 / k_{F} a$ in a trapped (solid line) and homogeneous (red dashed line) Fermi gas with a short-range potential, based on the decomposition given by Eq. (24) and Fig. 17.

number $N=66$.

The effects of a pseudogap or finite momentum pair contributions on thermodynamics is summarized by Fig. 19, where the entropy per particle is shown for a series of pairing strengths from BCS through BEC for a Fermi gas in a trap [152]. The black curve for $1 / k_{F} a=-2$ is close to a noninteracting Fermi gas, exhibiting a linear $T$ dependence at low $T$. In the opposite strong coupling BEC regime, the $1 / k_{F} a=3$ curve is close to the ideal Bose gas curve, above the BEC asymptote for $T_{c} / T_{F}, 0.518$. At high $T$ (but $\ll T^{*}$ ), it is easy to guesstimate from the figure that the entropy in the deep BEC regime is given roughly by half that for a free Fermi gas. The existence of finite momentum pairs allows a continuous evolution from the Fermi gas limit through the Bose gas limit, as the pairing strength increases. The presence of the trap inhomogeneity inevitably makes the situation more complicated that its homogeneous counterpart, leading to a power law $T$ dependence at low $T$ for all pairing strengths.

At unitarity, the distributions of the fermionic and bosonic components of the entropy are shown in the inset. At low $T$ in the broken symmetry, superfluid phase, the bosonic contribution $s_{b}(r)$ (blue curve) is nearly flat inside the superfluid core, and decays outside the core. On the other hand, the fermionic part, $s_{f}(r)$, comes mainly from the edge of the Fermi gas cloud, where the pairing gap becomes very small. The sum $s(r)$ has a peak at the trap edge as well. Considering the phase space factor $r^{2}$ in the trap integral, the behavior of the entropy $S$ at unitarity is dominated by the fermionic component at the trap edge. As the system evolves deep into the BEC regime, the fermionic part becomes negligible so that the bosonic part eventually dominates.

The above thermodynamics behavior has an immediate consequence. It can be used as a thermometry. It is well known that the temperature measurement in a Fermi gas is notoriously difficult. It is essentially impossible to measure the temperature at an arbitrary interaction strength. Measurement of the temperature in a Fermi gas without a population 


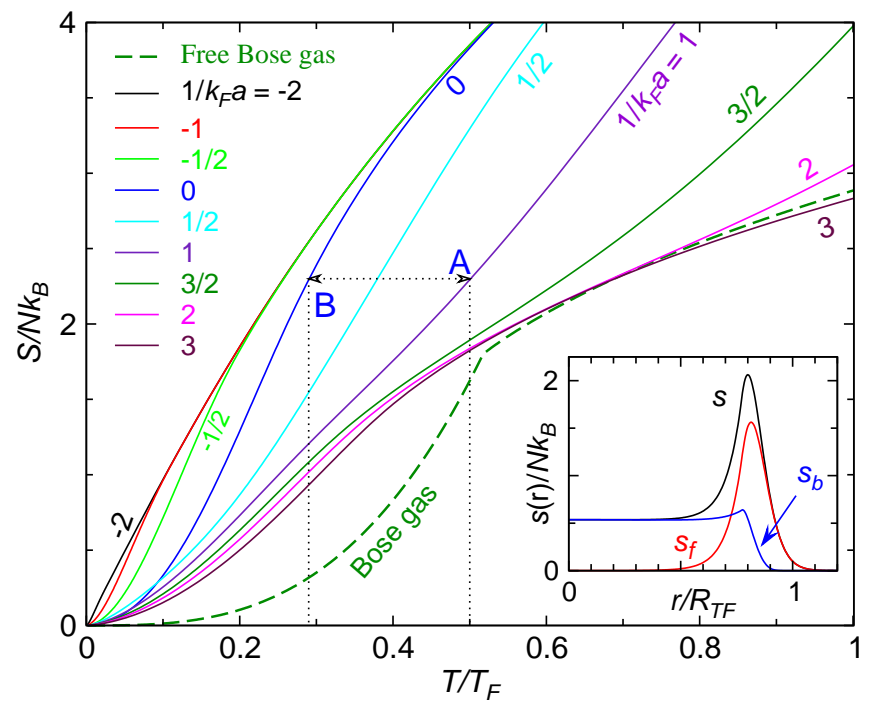

Figure 19. Entropy per atom as a function of $T$ for different pairing strengths from weak coupling BCS through strong coupling BEC in a harmonic trap. The dotted lines show an isentropic magnetic field sweep between $1 / k_{F} a=1$ and unitarity. For comparison, the dashed line represents the ideal Bose gas. The inset plots the spatial profile of total entropy $s(r)$ (black curve) and its fermionic $\left(s_{f}\right.$, red) and bosonic ( $s_{b}$, blue) component contributions at unitarity for $T=T_{c} / 4$. Here $T_{c}=0.27 T_{F}$. Reproduced from Ref. [153].

imbalance has been done successfully only in the BCS limit, deep BEC limit and at unitarity. In practice, it is convenient to connect the actual temperature at a given $1 / k_{F} a$ with the temperature in the non-interacting limit, using an adiabatic, isentropic magnetic field sweep. In other words, one can use the entropy in place of the temperature. As an example, the dotted lines in Fig. 19 shows how to connect the temperatures at $1 / k_{F} a$ and at unitarity.

As an application of the above pseudogap related thermometry, in Fig. 20 we plot the theoretically calculated phase diagram of ${ }^{40} \mathrm{~K}$ in a trap with an effective temperature $\left(T / T_{F}\right)^{0}$ measured adiabatically in the non-interacting limit, and compare with the experimental phase diagram from Jin's group $[45,154]$. The black and red curves are $T_{c}$ and the $N_{s} / N=$ 0.01 contour line, respectively. The experimental data show the contour plot of the condensate fraction. Given the large error bar in the data, we note that the overall trend of the experimental contour of $N_{0} / N=0.01$ and the theoretical line for $N_{s} / N=0.01$ are in good agreement [155]. One may also compare the $T_{c}$ curve with that shown in Fig. 15 to see directly the difference between $T_{c} / T_{F}$ and $\left(T_{c} / T_{F}\right)^{0}$ as an effect of the adiabatic isentropic sweep.

\section{EXPERIMENTAL EVIDENCE OF THE PSEUDOGAP IN ATOMIC FERMI GASES}

The concept of pseudogap was first introduced into atomic Fermi gases in Ref. [126]. It was not accepted and well understood by the AMO community, until more and more indi-

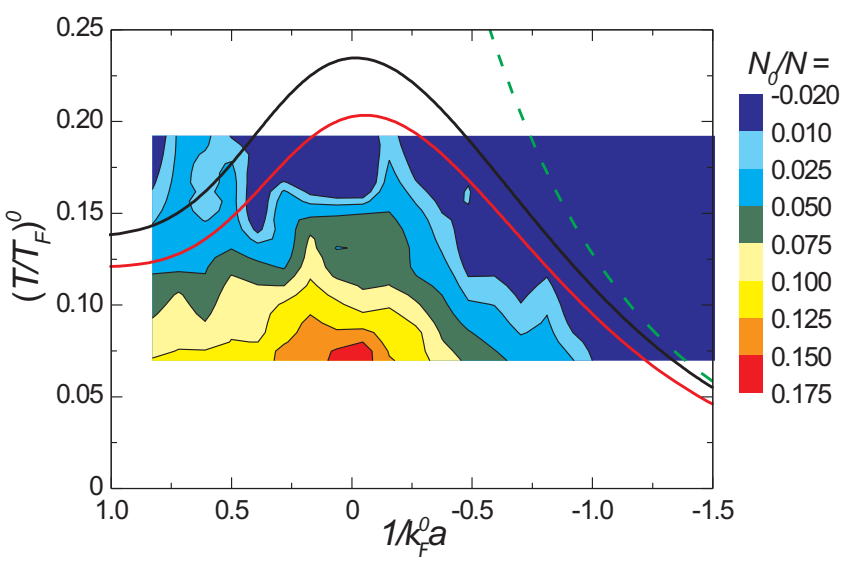

Figure 20. Phase diagram of ${ }^{40} \mathrm{~K}$. A contour plot of the measured condensate fraction $N_{0} / N$ as a function of $1 / k_{F}^{0} a$ and effective temperature $\left(T / T_{F}\right)^{0}$ in the noninteracting limit is compared with theoretically calculated contour lines at $N_{s} / N=0$ (at $T_{c}$, black curve) and 0.01 (red curve). Despite the large uncertainty in experimental data, the overall trend of the experimental contour of $N_{0} / N=0.01$ and the theoretical line for $N_{s} / N=0.01$ are in good agreement. The dashed line represents the naive BCS result $T_{c} / T_{F}^{0} \approx 0.615 e^{\pi / 2 k_{F}^{0} a}$. Here $k_{F}^{0} \equiv k_{F}$ and $T_{F}^{0} \equiv T_{F}$ are the global Fermi momentum and Fermi temperature, respectively. Reproduced from Ref. [154].

rect and direct experimental probes provided evidence for its existence. In this section, we shall present evidence of the pseudogap in atomic Fermi gases from various experiments, especially in the unitary regime.

Due to the extreme low $T$ and extreme small size as well as charge neutrality, the choice of experimental probes to ascertain the existence of the pseudogap is very limited for trapped Fermi gases. Typical condensed matter probes such as resistivity measurement, optical conductivity, penetration depth measurement, and angle-resolved photoemission spectroscopy (in the conventional sense) are not available. Therefore, one often has to resort to indirect measurements.

\section{A. Thermodynamics and density profiles}

Shown in Fig. 21 is the energy per atom for a unitary ${ }^{6} \mathrm{Li}$ Fermi gas. The lines are calculations of the present pairing fluctuation theory, while the symbols are experimental data from the Thomas group [49] at Duke University. The result for noninteracting Fermi gases serves as a calibration of the experimental measurement, where the Thomas-Fermi (TF) approximation works well. It is evident that the theory and experiment agree very well. It is worth mentioning that, in both the noninteracting and unitary cases, a finite trap depth as given by the experiment was used in order to arrive at the good agreement at high $T$. One of the most important messages one can read off the figure is that the unitary energy curve does not rise to that of the noninteracting curve until $T^{*} \approx 0.6 T_{F} \gg T_{c} \approx 0.29 T_{F}$ from the theory. This is a manifestation of the existence of a pseudogap above $T_{c}$ at unitarity, 


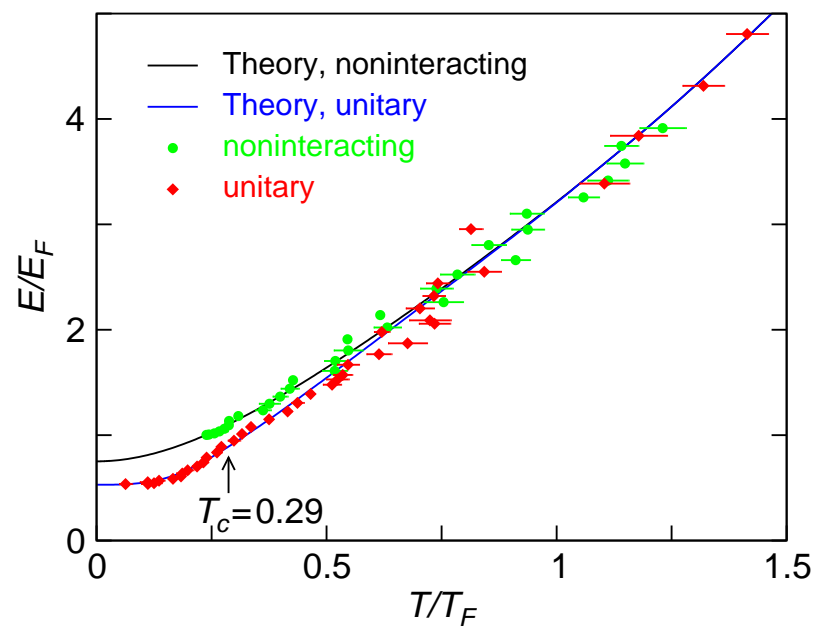

Figure 21. Comparison between theoretical calculations (lines) and experimental measurements of the energy per atom as a function of $T / T_{F}$ for noninteracting and unitary ${ }^{6} \mathrm{Li}$ gases. Here the temperature for the unitary case involves a temperature calibration [49]. The unitary and the noninteracting energy data do not merge until about $T^{*} \approx 0.6 T_{F}$. Reproduced from Ref. [49].

which helps to lower the energy.

If the energy curve $E(T)$ provides a signature of the pseudogap above $T_{c}$ at unitarity, the spatial density profile below $T_{c}$ may serve as indirect evidence of the pseudogap below $T_{c}$. In Fig. 22 we present a comparison of the one-dimensional density profile $\bar{n}(x)=\int \mathrm{d} z \mathrm{~d} y n(r)$ of a unitary Fermi gas between theory and experiment, at a temperature substantially below $T_{c}$. The agreement is good. There is no sign of the kink behavior at the edge of the superfluid core in the data [157]. We stress that such a good agreement is not expected for a mean-field theory $[148,149]$ or a theory that exhibits non-monotonic dependence in radius or temperature [116].

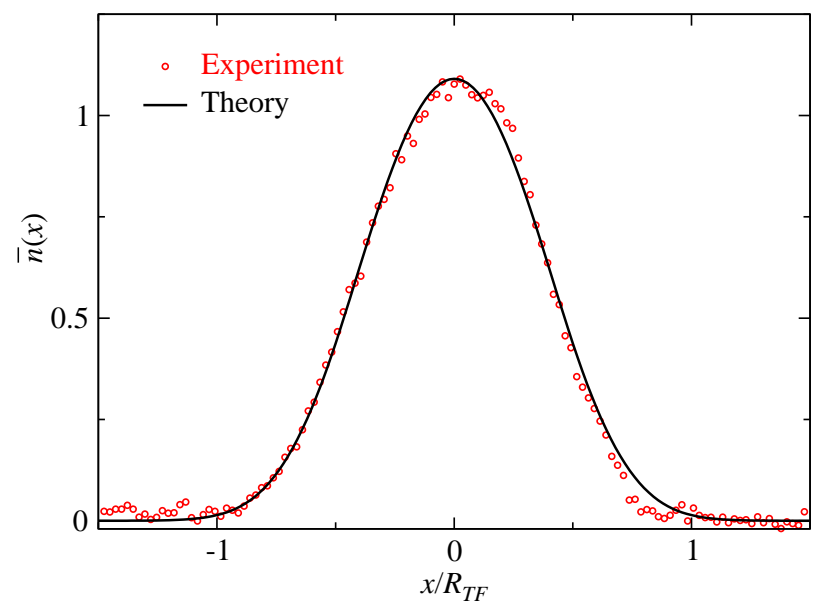

Figure 22. Comparison between theoretical calculations (black solid line) and experimental measurements (red circles) of the onedimensional density profile of a unitary ${ }^{6} \mathrm{Li}$ gas at $T=0.19 T_{F} \approx$ $0.7 T_{c}$, in the superfluid phase. Reproduced from Ref. [156].

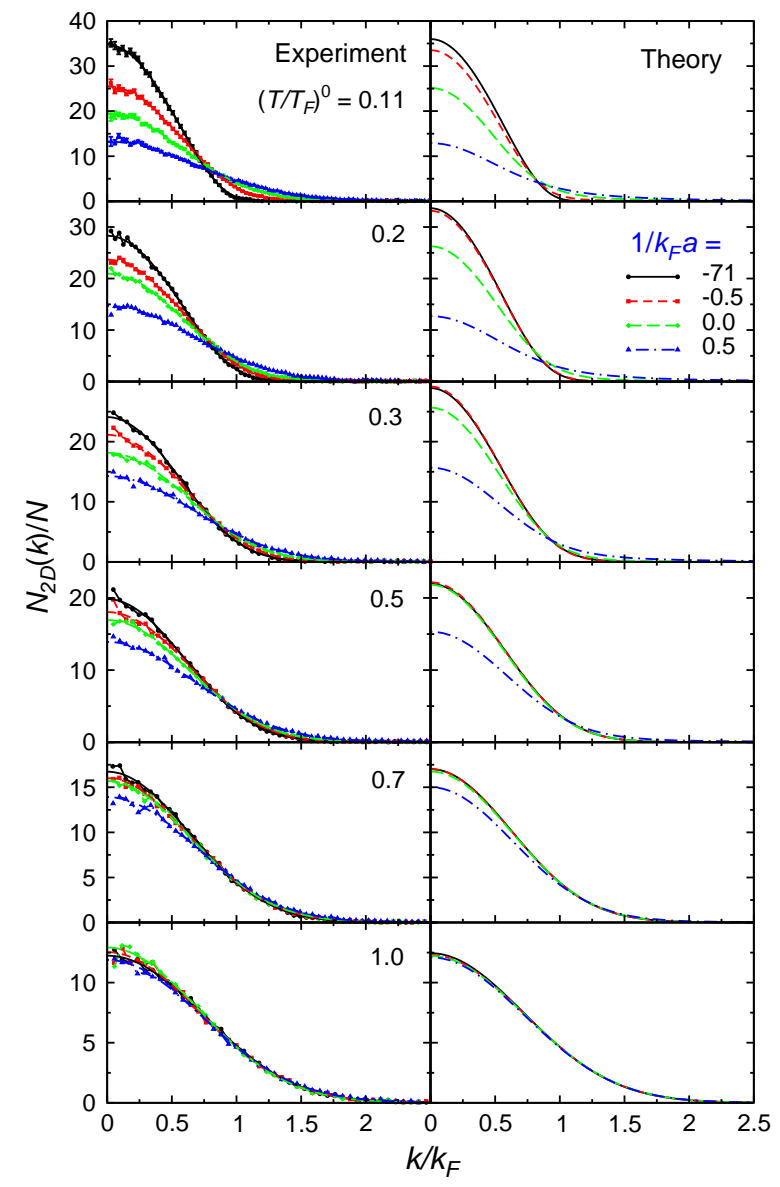

Figure 23. Temperature evolution of the 2D momentum distribution $N_{2 D}(k)$ of a ${ }^{40} \mathrm{~K}$ gas in a trap with different pairing strengths from noninteracting through near-BEC cases. The effective temperature in the noninteracting limit, $\left(T / T_{F}\right)^{0}$, is labeled. Reproduced from Ref. [158].

As shown in Fig. 17, the pseudogap or finite momentum pair contributions are essential in arriving at such a smooth density profile.

\section{B. Momentum distribution}

The presence of a pseudogap necessarily has an important consequence on the momentum distribution $N_{k}$ of the Fermi gases. Without a pseudogap, $N_{k}$ would behave very much like a noninteracting Fermi gas at a given temperature. In contrast, it will be spread to a larger range in the momentum space, according to

$$
\begin{aligned}
N_{2 D}(k) & =\int \frac{\mathrm{d} k_{z}}{2 \pi} N_{k}=\int \frac{\mathrm{d} k_{z}}{2 \pi} \mathrm{d}^{3} r n_{k}(r), \\
n_{k}(r) & =1-\frac{\xi_{\mathbf{k}}}{E_{\mathbf{k}}}+2 \frac{\xi_{\mathbf{k}}}{E_{\mathbf{k}}} f\left(E_{\mathbf{k}}\right) .
\end{aligned}
$$

In Fig. 23, we present the 2D momentum distribution $N_{2 D}(k)$ at a series of temperatures from below to far above $T_{c}$ for different pairing strengths from BCS through (near-)BEC 


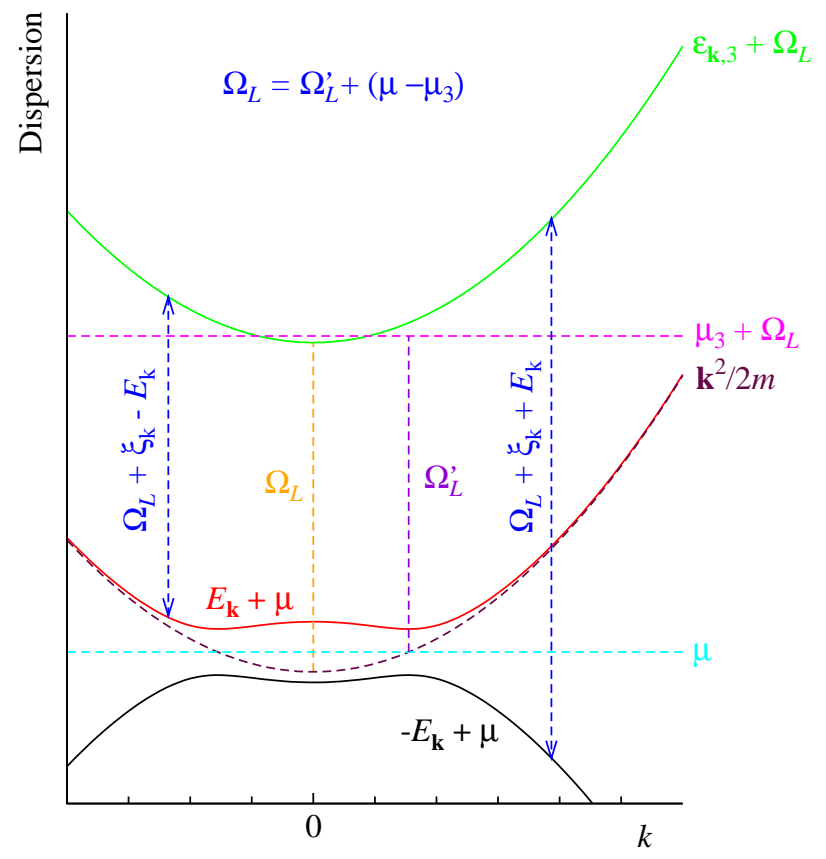

Figure 24. Energy levels in an RF transition. $\Omega_{L}$ is the RF frequency for exciting a free atom from hyperfine level 2 (maroon dashed curve) to level 3 (green solid curve). $\Omega_{L}^{\prime}$ is the same energy but measured relative to the respective chemical potentials. The black and red solid curves are the dispersion of the condensed and thermally excited quasiparticle branches of a paired atom in level 2, with energy level given by $\mp E_{\mathbf{k}}+\mu$, respectively. Reproduced from Ref. [159].

regimes. The left and right columns are from experiment and theory, respectively. The comparison reveals a good agreement between them. The most important message here is that the $N_{2 D}(k)$ curves differs significantly for different pairing strengths at $\left(T / T_{F}\right)^{0}=0.3$ and 0.5 , above $T_{c}$, and they do not merge until $\left(T / T_{F}\right)^{0}>0.7$ where the (pseudo-) excitation gap disappears. Like the $\left(T / T_{F}\right)^{0}=0.11$ case, the difference between the curves for different pairing strengths is caused by the presence of the (pseudo-)gap.

\section{C. (Momentum integrated) radio frequency spectroscopy}

Among various experimental techniques, radio-frequency (RF) spectroscopy $[47,160,161]$ is arguably the most direct probe for the existence of an excitation gap. The basic physics of a RF process is shown in Fig. 24. Atoms in hyperfine levels 1 and 2 are subject to the pairing interaction, whereas atoms in level 3 are free of such pairing. Therefore, by exciting an atom from level 2 to an unoccupied level 3, one can tell how much extra energy is needed in addition to the hyperfine level splitting. This extra energy, referred to as detuning, provides a measure of the "binding energy" of the level 2 atoms due to interactions. To the lowest order, the RF current is given by

$$
I(\nu)=-\left.\frac{1}{2 \pi} \sum_{\mathbf{k}} A(\mathbf{k}, \omega) f(\omega)\right|_{\omega=\xi_{\mathbf{k}}-\nu},
$$

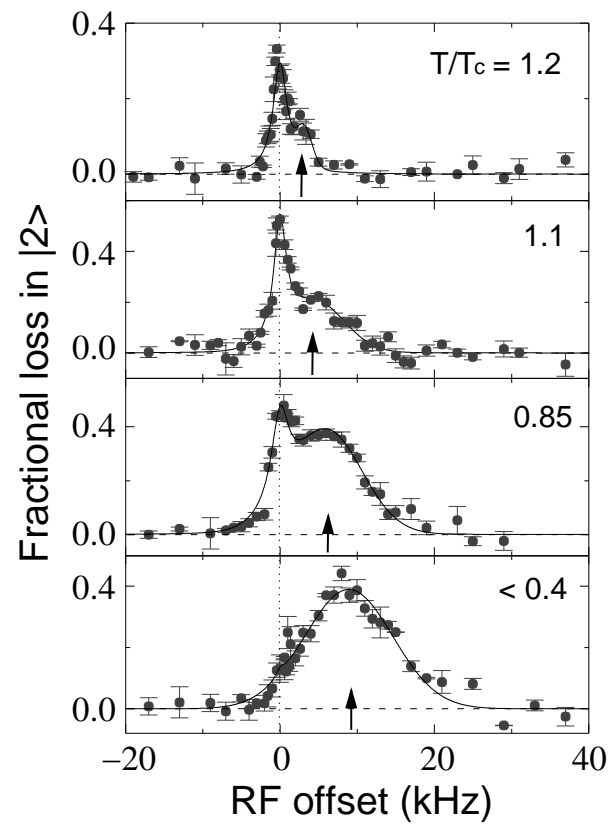

Figure 25. RF spectra of a unitary ${ }^{6} \mathrm{Li}$ gas for different temperatures. The solid lines are fits to guide the eye. The vertical dotted line marks the atomic transition, and the arrows indicate the peak location of the pairing signal. The original effective temperature $T^{\prime} / T_{F}$ has been converted isentropically to the real (reduced) temperature $T / T_{c}$ using the entropy data shown in Fig. 19. Reproduced from Ref. [47].

where $\nu$ is the detuning, $A(\mathbf{k}, \omega)$ is the spectral function for level 2 atoms, and we have set the RF matrix element to unity. As is well known, when level 2 atoms are paired with level 1 atoms, the spectral function $A(\mathbf{k}, \omega)$ consists of two branches, the condensed and thermally excited quasiparticle branches, represented in Fig. 24 by the black and red solid curves, respectively. The thermal branch corresponds to negative detuning, and will not be observable for either very low or very high $T$. The former case will be suppressed by the Fermi function $f(\omega)$, while the high temperature will destroy the pairing in the later case. When interactions exist between level 3 atoms and level 1 atoms, then we have a final state effect, which will also affects the RF spectrum [162-167].

Shown in Fig. 25 is the earliest report on the RF spectroscopy measurement of a unitary ${ }^{6} \mathrm{Li}$ gas at different temperatures. The fractional loss is proportional to $A(\mathbf{k}, \omega)$ and the RF offset is the detuning $\nu$. The effective temperature $T^{\prime} / T_{F}$, measured in the BEC limit after an isentropic sweep, has been converted to the real temperature $T / T_{c}$, using the calculated entropy data shown in Fig. 19. The important feature of this figure is the double peak structure, with one narrow sharp peak at zero detuning, and a broad peak with a positive detuning. The narrow peak can be easily attributed to the transition from the free level 2 atoms, found at the edge of the trap. On the other hand, the broad peak has been associated with paired level 2 atoms. The trap inhomogeneity necessarily leads to a distribution in the pairing gap, and thus the broadness of the $\mathrm{RF}$ signal. The phase space factor $r^{2}$ in the trap integral determines that the pairing signal will be peaked at an intermediate 


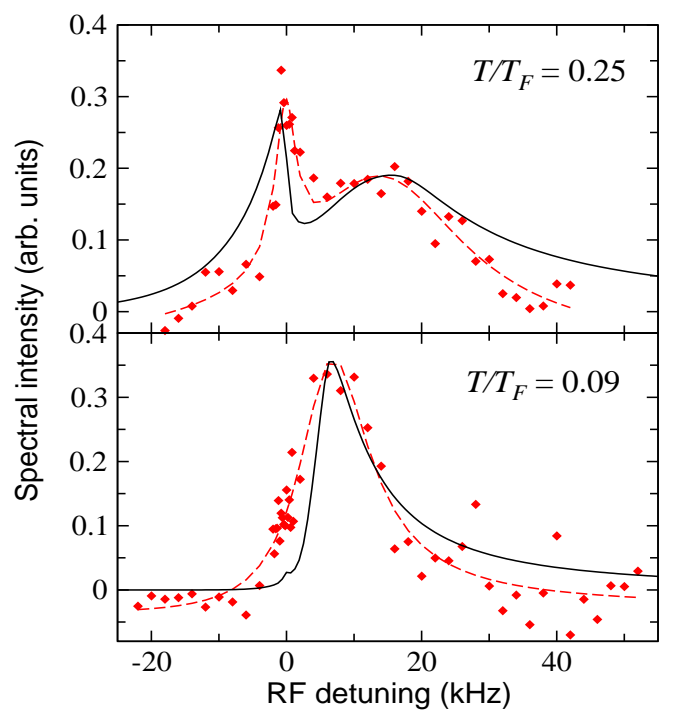

Figure 26. Comparison of calculated RF spectra (solid curves, $T_{c} \approx$ $0.29 T_{F}$ ) with experiment (symbols) in a harmonic trap calculated at $822 \mathrm{G}$ for the two lower temperatures. The temperatures were chosen based on Ref. [47]. The dashed lines are a guide to the eye. Reproduced from Ref. [173].

radius. At unitarity, for a given gap $\Delta$, the detuning would be a momentum average of

$$
\nu=E_{\mathbf{k}}+\xi_{\mathbf{k}} \geq \sqrt{\mu^{2}+\Delta^{2}}-\mu<\Delta,
$$

with the spectral weight given by the integrand of Eq. (26), namely, the momentum dependent RF current. While quantitatively, the location of the broad pairing peak does not give directly the pairing gap, qualitatively, its presence is a signature of pairing. As revealed by Fig. 25, the broad peak can already be seen above $T_{c}$ at $T / T_{c}=1.2$. The total spectral weight under the broad peak as well as the detuning for this peak increases as $T$ decreases further. Deep in the superfluid phase at $T / T_{c}<0.4$, the free atom peak is essentially gone; all level 2 atoms are paired, and the pairing peak detuning reaches its maximum.

Recent experiment[168] and QMC results [169-171] suggest that for ${ }^{6} \mathrm{Li}$ at unitarity, the transition temperature $T_{c} / T_{F} \approx 0.17$, substantially lower than 0.29 predicted in the present theory. This further substantiates the existence of a pseudogap above $T_{c}$.

The experimental result of Ref. [47] was interpreted successfully $[172,173]$ using the present pairing fluctuation theory soon after its publication. In Fig. 26, we present a comparison of calculated RF spectra (solid curve, $T_{c} \approx 0.29 T_{F}$ ) with experiment (symbols) in a harmonic trap calculated for ${ }^{6} \mathrm{Li}$ at $822 \mathrm{G}$ (on the BEC side of the Feshbach resonance at $834 \mathrm{G}$ ) for the two lower experimental temperatures. The overall agreement is satisfactory, which can be further improved by including the final state effect [159]. While our focus remains on qualitative evidence of the pseudogap, we shall not go into details about the final state effect. Interested readers may find further information in Refs. [159, 162, 164167].

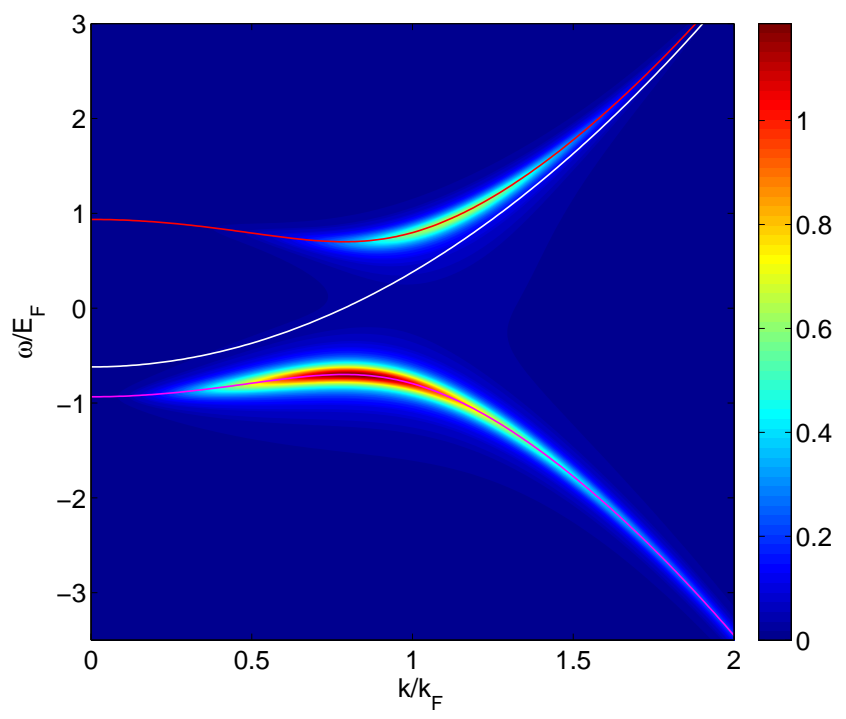

Figure 27. . Contour plots of the occupied spectral intensity at unitarity in a homogeneous Fermi gas for $T / T_{c} \approx 1.9$. The population of the two branches are determined self-consistently. The white curve represents the dispersion of unpaired atom. Reproduced from Ref. [176].

\section{Momentum resolved radio frequency spectroscopy}

Despite the very intuitive picture about the double peak structure in the RF spectra, the momentum integration has caused some disputes regarding the origin of the double peak structure $[160,174]$, and thus the physical interpretation about the RF spectroscopy measurements. This has a lot to do with the final state effect, first noticed by Muller and coworkers [166]. The lack of simple relation between the pairing gap size and the pairing peak location in the RF spectra has made it difficult to extract the gap from the data quantitatively.

A great step forward was made by Jin and coworkers [175], who performed momentum resolved RF (MRRF) spectroscopy measurement for the first time, in a ${ }^{40} \mathrm{~K}$ gas. The RF current is given by the integrand of Eq. (26). It turns out that the MRRF spectroscopy is equivalent to the ARPES [61, 91], which is a very important and useful tool in condensed matter physics. Further simplification comes from the fact that there is no final state effect in a ${ }^{40} \mathrm{~K}$ gas. This makes the interpretation of the MRRF spectra relatively simple and unambiguous. In Fig. 27, we present the contour plot of the occupied spectral intensity in the $\omega-k$ plane, for a homogeneous 3D Fermi gas at unitarity at $T / T_{F}=0.5$. The two branches corresponding to the condensed and thermally excited quasiparticles shown in Fig. 24 are clearly visible. For comparison, the white curve shows the dispersion of a free atom, with the same chemical potential. The particle-hole mixing as a pairing effect is evident, as manifested by the avoided crossing and back-bending of both the lower and upper branches. This back-bending takes place at $k=\sqrt{2 m \mu}<k_{F}$. To see the upper branch clearly, one needs to have relatively high temperature which is comparable with $\Delta$.

Ideally, one would like to have a homogeneous system. Un- 

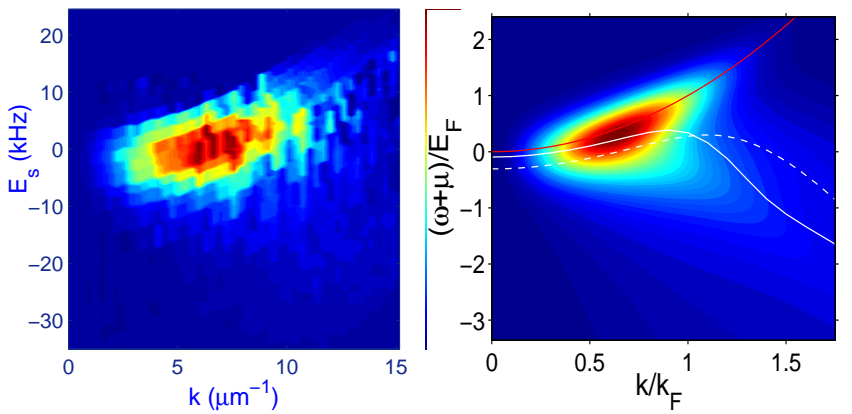

Figure 28. Comparison between experiment (left) and theory (right) of the contour plots of the occupied spectral intensity in a unitary Fermi gas in a trap at $T / T_{c} \approx 1.1$. The red curve represents the free atom dispersion. A large amount of spectral weight has been shifted from a free atom peak to a paired atom peak (downward dispersing curve). Here the intensity increases from dark blue for 0 to dark red for the maximum, and the solid and dashed white lines indicate the loci of the peaks in the energy distribution curves from theory and experiment, respectively. The single particle energy $E_{s}$ is equivalent to $\omega+\mu$, the energy measured relative to the bottom of the band. Reproduced from Refs. [175, 176].

fortunately, a trap potential is necessary in order to hold the Fermi gas together. This complicates the otherwise very simple interpretation of the RF spectra.

In Fig. 28, we present a comparison of the key result on the spectral intensity map between experiment (left) and theory (right) for a unitary Fermi gas above $T_{c}$ at $T / T_{c}=1.1$. The similarity between the two panels is obvious. As can be seen, a large fraction of the spectral weight has been shifted from the free atom branch to the paired atom branch. Indeed, at high $T$ where pairing effect is negligible, the spectral weight concentrates on the free particle dispersion (not shown). As the temperature decreases, a second (downward dispersing) branch emerges. This lower branch is associated with the breaking of a pair and necessarily contains trap averaging effects. With decreasing temperature, the intensity map first bifurcates and eventually at very low $T$ becomes dominated by the lower branch, when essentially all atoms are paired.

From Fig. 28, such bifurcation and downward dispersion already take place above $T_{c}$, indicating unambiguously that a pseudogap exists in the unitary Fermi gas. To extract this downward dispersion, the energy distribution curves (EDCs) have been fitted to a single Gaussian function experimentally. This leads to the white dashed curve in the right panel. The white solid dispersion curve is obtained theoretically following the same procedure. A BCS-like fit to this dispersion can be used to determine the pairing gap, as has been done in Ref. [175], as $E_{s}=\mu-\sqrt{\xi_{\mathbf{k}}^{2}+\Delta^{2}}$. The best fit to the experimental dispersion yields $\Delta=9.5 \mathrm{kHz}$, comparable to $E_{F}$. The agreement between experiment and theory is reasonably good, despite the trap inhomogeneity. The white dashed experimental curve back bends at $k>k_{F}$, in contradiction to what is expected on physical ground at unitarity. This is mainly caused by the low experimental resolution and the incorrect single-Gaussian function fitting procedure. Our theory predicts double peaks in the EDC curves for $k \geq k_{F}$, and

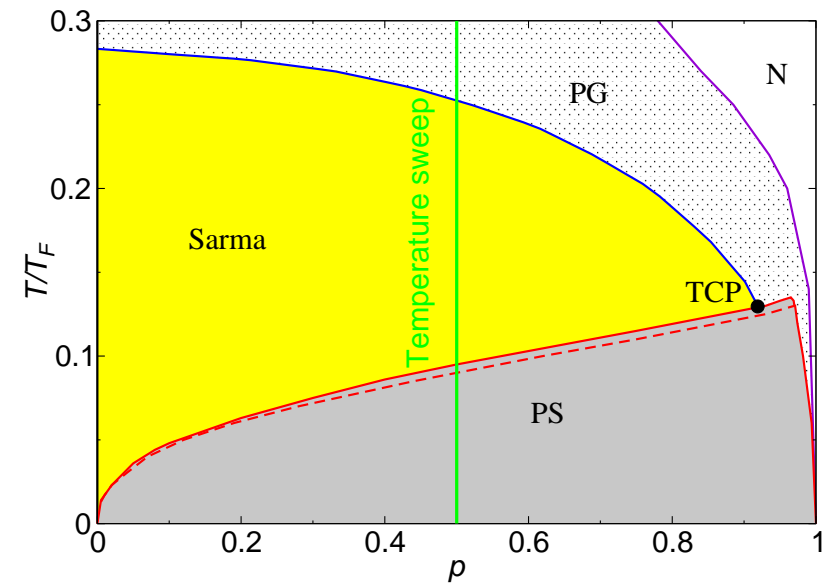

Figure 29. Calculated phase diagram of a population imbalanced Fermi gas in a trap at unitarity. Here "PS" stands for phase separation, "Sarma" for polarized Sarma superfluid, "PG" for pseudogapped normal state, "N" for unpaired normal state, and "TCP" for tricritical point. Reproduced from Ref. [133].

this has been confirmed by careful inspection of the experimental data [176]. Further improved experimental data [177] have led to a dispersion much closer to the theoretical result. The observation of a pseudogap has also been confirmed by Refs. [178, 179].

It should be mentioned that via the simple approximation Eq. (5), the present pairing fluctuation theory has demonstrated in a simple, analytic way that a pseudogap necessarily exists when the pairing interaction is strong. For NSR-based theories, due to the inconsistency between the gap equation, which contains no pairing fluctuation contributions, and the number equation, one would have to extract the pseudogap from the renormalized spectral function in a cumbersome way. In this sense, the numerical route of Strinati et al [180, 181] can be viewed simply as a confirmation of our analytically result.

\section{E. Population imbalanced Fermi gases}

In this subsection, we provide evidence for the existence of a pseudogap in population imbalanced Fermi gases. For extension of the present pairing fluctuation theory to the case of population imbalance, we refer the readers to Refs. [132134]

Shown in Fig. 29 is the calculated phase diagram of a population imbalanced Fermi gas in a trap at unitarity. The overall polarization, $p=\left(N_{\uparrow}-N_{\downarrow}\right) / N$, is different from its local counterpart. At low $T$, phase separation (PS) takes place, where a BCS superfluid core of an equal spin mixture at the trap center is surrounded by polarized Fermi gases. Above the PS phase, there exist intermediate temperature superfluids, which is referred to as Sarma superfluid, for which the local spin polarization penetrates all the way into the trap center. Above the Sarma phase, there is a pseudogap phase (PG) where pseudogap exists without superfluidity, before the sys- 

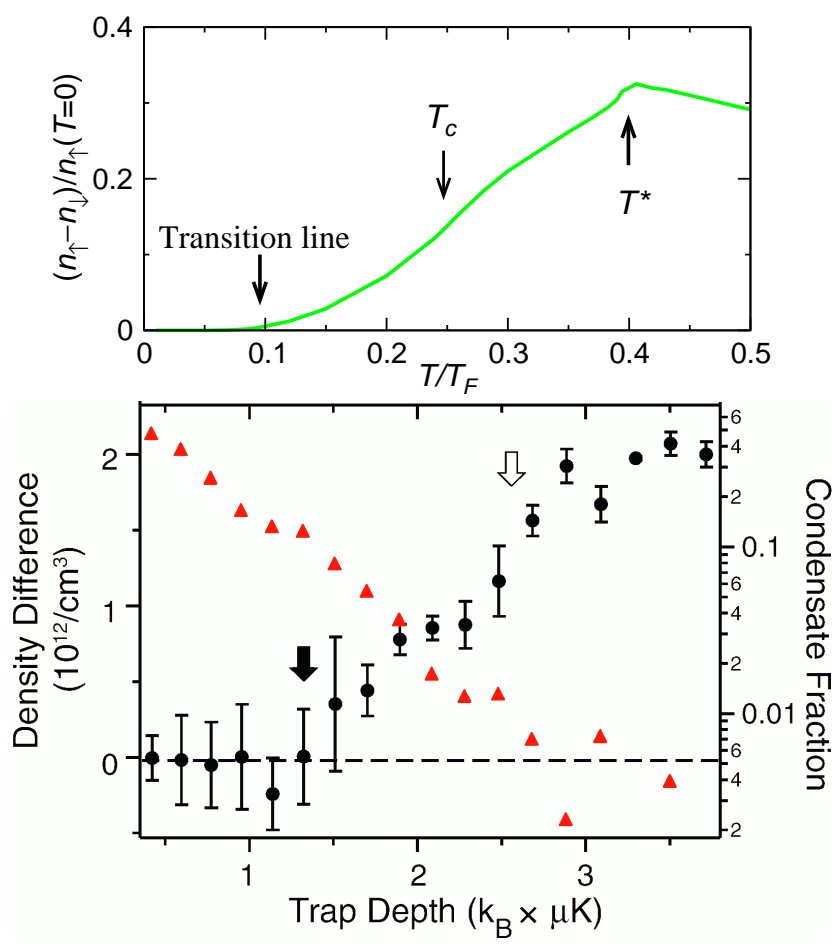

Figure 30. Upper panel: Relative density difference at the trap center at low $T$ as a function of $T / T_{F}$ at $p=0.5$, i.e., along the vertical green line in Fig. 29. The three arrows indicate the PS/Sarma boundary, the Sarma superfluid/PG transition $T_{c}$, and the PG/N crossover temperature $T^{*}$, respectively. Lower panel: Experimental data from MIT, showing the density difference (black solid circles, left axis) at the trap center of a unitary Fermi gas at $p=0.5$, along with the condensate fraction (red triangles, right axis), as a function of trap depth, which is proportional to $T$. The solid and empty arrows indicate the PS/Sarma transition temperature and superfluid $T_{c}$, respectively. Reproduced from Refs. [133, 182].

tem becomes unpaired normal state $(\mathrm{N})$ at high $T$.

The behavior of the polarization at the trap center, $\left(n_{\uparrow}-\right.$ $\left.n_{\downarrow}\right) / n_{\uparrow}(T=0)$, in a temperature sweep at $p=0.5$, is shown in the upper panel of Fig. 30. An important feature here is that its evolution across $T_{c}$ is smooth, without a clear signature of the superfluid transition. A downturn is predicted at the crossover temperature $T^{*}$, where the pseudogap becomes negligible. We emphasize that this smooth evolution across $T_{c}$ is a consequence of the fact that the total excitation gap is continuous across $T_{c}$. This feature has been verified by experimental data from the Ketterle group [182], as shown in the lower panel. Note that the experimental trap depth is proportional to the temperature. The agreement between experiment and theory is remarkable. Therefore, we conclude that the experimental data have provided strong support for the existence of a pseudogap above $T_{c}$.

\section{F. Dispute against the existence of a pseudogap}

Despite the definitive evidence of the pseudogap from various experiments, especially the MRRF spectroscopy measure- ments, there have still been some disputes against the existence of the pseudogap from thermodynamics measurement [183, 184]. Especially, in Ref. [183], Salomon and coworkers reported "a $T^{2}$ dependence of the pressure with temperature", and thus claimed that "This behavior is reminiscent of a Fermi liquid, and indicates that pseudogap effects expected for strongly interacting Fermi superfluids do not show up at the thermodynamic level within our experimental precision." However, this cannot be used as evidence against the existence of a pseudogap, because the macroscopic quantity pressure used in their equation of state (EOS) involves a trap and momentum integration over all microscopic states. There are many possible microscopic states which can produce the same macroscopic thermodynamic quantities after integration. This is a many-to-one mapping. For example, within the BCS mean-field theory, the relation between pressure and energy of a Fermi gas with a contact potential at unitarity is given by $p / E=2 / 3$, exactly the same as that for a noninteracting Fermi gas (which exhibits an ideal Fermi liquid behavior). In fact, their key experimental data were taken at $(T / \mu)^{2}>0.1$, or equivalently, $T / \mu>0.3$. This is far from being a low $T$ regime, where one can talk about power law dependence. At such a high temperature, it is not particularly useful to extract its power law dependence on $T$. The pressure calculated with a pseudogap would follow a similar $T$ dependence in this temperature regime, just like that of the energy (per particle), $E(T)$.

\section{WHERE TO LOOK FURTHER FOR THE PSEUDOGAP}

\section{A. Effects of particle-hole fluctuations}

As in most other theories of BCS-BEC crossover, e.g., the NSR-based theories, the particle-hole channel has been dropped in the treatment of the present pairing fluctuation theory. This is justified in the context of superconductivity, where the particle-hole channel mainly contributes to a change in the chemical potential, which can be taken from experiment. In addition, superfluidity and pairing concerns primarily the particle-particle channel. In many cases, the pairing interaction strength is not precisely known, and thus may be treated as a fitting parameter. Nevertheless, when the pairing interaction strength is indeed known precisely, one may need to consider the effect of particle-hole fluctuations.

In the weak coupling limit, the contribution of particlehole fluctuations was first considered by Gor'kov and MelikBarkhudarov (GMB) [185] to the leading order. They found that both $T_{c}$ and zero temperature gap are suppressed by a big factor of $(4 e)^{1 / 3} \approx 2.22$. A few others have recently considered particle-hole fluctuations within the context of Fermi gases and BCS-BEC crossover [186-189]. In Ref. [190], the present pairing fluctuation theory is extended to include the particle-hole channel in such a fashion that the $T$-matrices of both the particle-particle channel and the particle-hole channel intertwine with each other and are treated self-consistently. The main result is that in the BCS through unitary regime where the chemical potential $\mu>0$, particle-hole fluctuations 


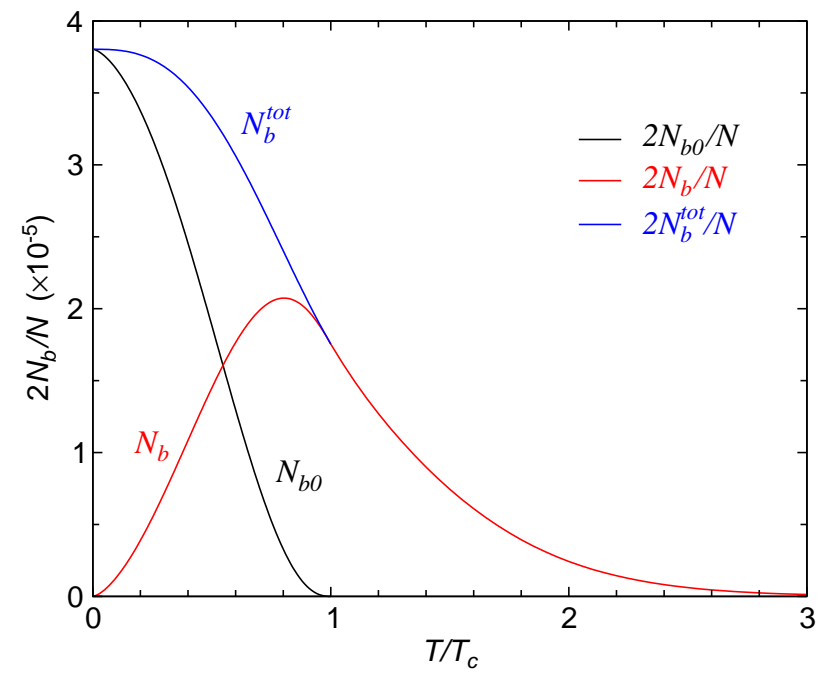

Figure 31. Closed-channel fraction as a function of $T / T_{c}$ at unitarity for $T_{F}=0.4 \mu \mathrm{K}$ for ${ }^{6} \mathrm{Li}$ in a harmonic trap. The black, red, and blue curves are the condensed $\left(2 N_{b 0} / N\right)$, noncondensed $\left(2 N_{b} / N\right)$ and total $\left(2 N_{b}^{t o t} / N\right)$ fractions, respectively. Here $T_{c}=0.273 T_{F}$. Reproduced from Ref. [191].

cause an effective reduction of the pairing strength. In particular, the unitary limit is shifted towards the BEC regime, to $1 / k_{F} a \approx 0.35$. The original maximum $T_{c}$ at unitarity (see Fig. 9) has now occurred at the new location. This seems to be in good agreement with the QMC result from Ref. [170], which reported a maximum $T_{c} / E_{F} \approx 0.25$ around $1 / k_{F} a=0.47$. Depending how the interaction parameter is determined, this seems to suggest that one may need to consider looking for the pseudogap around the new unitary limit in future experiments. Further details of the effect of particlehole fluctuations may be found in Ref. [190].

\section{B. Widespread pseudogap phenomena}

There are widespread pseudogap related phenomena in ultracold Fermi gases. In this section, we shall only name a few examples, instead of giving a full search.

For a wide Feshbach resonance such as the widely studied resonances in ${ }^{6} \mathrm{Li}$ and ${ }^{40} \mathrm{~K}$, the closed channel fraction has turned out to be closely related to the pseudogap. In Fig. 31, we show the closed-channel fraction as a function of $T$ for a unitary Fermi gas, calculated using a two-channel version of the present pairing fluctuation theory [191]. Here the black $\left(N_{b 0}\right)$ and red $\left(N_{b}\right)$ curves stand for the condensed and thermal part of the closed-channel molecules, while the blue curve $\left(N_{b}^{t o t}\right)$ stands for the sum. They are proportional to $\Delta_{s c}^{2}$, $\Delta_{p g}^{2}$, and $\Delta^{2}$, respectively. At low $T$, the calculated fraction $2 N_{b}^{t o t} / N$ as a function of pairing strength is in good quantitative agreement with experiment [191, 192]. It is known that at unitarity, pairing can exist only due to many-body effect. Above $T_{c}$, should there be no pairing (or equivalently, pseudogap), the closed-channel fraction would drop to zero due to the inter-channel coupling. Therefore, detection of the

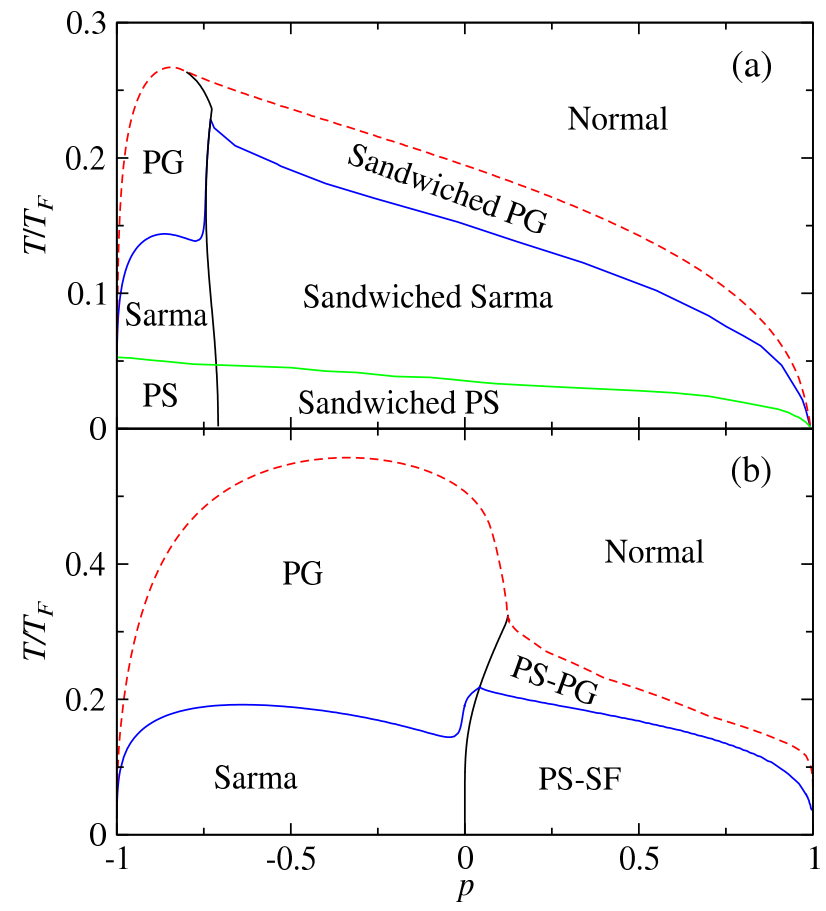

Figure 32. $T-p$ Phase diagram of a ${ }^{6} \mathrm{Li}^{-}{ }^{40} \mathrm{~K}$ mixture in a harmonic trap at (a) unitarity and (b) $1 / k_{F} a=0.5$, with $\omega_{\uparrow}=\omega_{\downarrow}$. The solid lines separate different phases, and the (red) dashed line is approximated by mean field calculations. We choose the population imbalance $p>0$ when ${ }^{40} \mathrm{~K}$ is the majority. Here "PG" and "PS" indicate pseudogapped normal state and phase separation, respectively, and "SF" stands for superfluid. The PS-PG and PS-SF phase has an "inverted" two-shell structure, with a normal gas of the majority heavy atoms at the trap center, surrounded by a superfluid or pseudogapped paired Fermi-Fermi mixture. Reproduced from Ref. [135].

closed-channel fraction above $T_{c}$ should provide a direct measurement of the pseudogap.

The pseudogap phenomena can be found not only in equal mass two component Fermi gases, but also in a Fermi-Fermi mixture, with a strong mass imbalance. In Fig. 32, we present a phase diagram for a ${ }^{6} \mathrm{Li}^{-}{ }^{40} \mathrm{~K}$ mixture in a harmonic trap (a) at unitarity and (b) in the near-BEC regime $1 / k_{F} a=0.5$, with $\omega_{\uparrow}=\omega_{\downarrow}$. Here $\omega_{\sigma}$ is the angular frequency of the spin dependent trapping potential. The convention is such that $p>0$ when the heavy species is the majority. The "PS", "Sarma" and "PG" phase in Fig. 32(a) at unitarity is similar to that in Fig. 29. As usual, at the highest temperature, the system behaves like a mix of uncorrelated normal Fermi gases. Otherwise, the dominant part of the phase diagram at unitarity is a sandwiched three-layer shell structure, for which the inner and outer shells are normal Fermi gases, while the mid-shell is either a BCS, Sarma superfluid or a PG normal state, for a temperature from low through high. In the near BEC case in Fig. 32(b), the PS phase for $p<0$ is no longer stable and the PG and Sarma regions expands substantially. For $p>0$, the outer shell of a majority Fermi gas has disappeared so that the system becomes an "inverted" two-shell structure, with a majority Fermi gas at the trap center, surrounded by a su- 
perfluid or pseudogapped paired Fermi-Fermi mixture in the outer shell. Such two-shell or three-shell structures and the local density profiles may be probed using the in situ phasecontrast imaging and 3D image reconstruction technique as in Ref. [182]. One may also use vortex lattices [50] to detect the (sandwiched) PS and Sarma state, so as to distinguish the superfluid and pseudogapped phases. The paired state may also be detected using a Bragg spectroscopy technique [193, 194], which may also be able to distinguish condensed versus noncondensed pairs.

It should be emphasized that the sandwiched PG and the PS-PG phases are both very unusual and very interesting. The associated phase separation involves pseudogapped normal state rather than a superfluid state. Such phase separations have never been predicted or reported before in the literature.

There are many other experiments and physical quantities which exhibit pseudogap phenomena. For example, atomic Fermi gases on optical lattices will be another realm to search for the pseudogap in the future, despite that experiment on optical lattices falls far behind theory. Another realm is $2 \mathrm{D}$ Fermi gases, since low dimensionality intrinsically enhances fluctuations, including the pseudogap related pairing fluctuations. It is expected that more support for the existence of a pseudogap will come up as new experiments become available.

\section{SUMMARY}

In summary, we have given a review of the study of the pseudogap physics in atomic Fermi gases, and presented a se- ries of experimental evidence of the existence of a pseudogap in Fermi gases, especially in the unitary regime. In this context, we have introduced a pairing fluctuation theory, and have shown that it thus far has addressed successfully multiple atomic Fermi gas experiments. In particular, the momentum resolved radio frequency spectroscopy measurement has provided the most direct probe and the most convincing evidence of the pseudogap. Since the existence of a pseudogap is a natural consequence of the present theory, and most competing theories do not have a pseudogap in their fermion self energy in a self-consistent fashion, the experimental evidence of a pseudogap can be viewed as a strong support for this theory. Given the analogy between superfluidity in Fermi gases and superconductivity in high $T_{c}$ superconductivity, we argue that the present pairing fluctuation theory for the pseudogap is also a strong candidate for high $T_{c}$ superconductivity.

\section{ACKNOWLEDGMENTS}

This work is supported by the National Basic Research Program of China (under grants No. 2011CB921303 and No. 2012CB927404), the Natural Science Foundation of China (No. 10974173 and No. 11274267), and of Zhejiang Province (No. LZ13A040001).
[1] Q. J. Chen, J. Stajic, S. N. Tan, and K. Levin, BCS-BEC crossover: From high temperature superconductors to ultracold superfluids, Phys. Rep., 2005, 412: 1.

[2] I. Bloch, J. Dalibard, and W. Zwerger, Many-body physics with ultracold gases, Rev. Mod. Phys., 2008, 80: 885.

[3] J. M. Maldacena, The large $N$ limit of superconformal field theories and supergravity, Adv. Theor. Math. Phys., 1998, 2: 231, see also e-print arXiv:hep-th/9711200v3.

[4] E. Witten, Anti de sitter space and holography, Adv. Theor. Math. Phys., 1998, 2: 253.

[5] O. Aharony, S. S. Gubser, J. Maldacena, H. Ooguri, and Y. Oz, Large $N$ field theories, string theory and gravity, Phys. Rep., 2000, 323: 183.

[6] M. Cubrović, J. Zaanen, and K. Schalm, String theory, quantum phase transitions, and the emergent fermi liquid, Science, 2009, 325: 439.

[7] T. Timusk and B. Statt, The pseudogap in high-temperature superconductors: An experimental survey. Rep. Prog. Phys., 1999, 62: 61.

[8] J. R. Schrieffer, Theory of Superconductivity, 3rd ed. (Perseus Books, Reading, MA, 1983).

[9] S. N. Bose, Plancks gesetz und lichtquantenhypothese, Z. Phys., 1924, 26: 178.

[10] A. Einstein, Quantentheorie des einatomigen idealen gases, Sitzungsberichte der Preussischen Akademie der Wis- senschaften, 1925, 1: 3.

[11] L. Pitaevskii and S. Stringari, Bose-Einstein Condensation (Oxford, New York, 2003).

[12] C. J. Pethick and H. Smith, Bose-Einstein Condensation in Dilute Gases (Cambridge University Press, Cambridge, 2002).

[13] D. M. Eagles, Possible pairing without superconductivity at low carrier concentrations in bulk and thin-film superconducting semiconductors, Phys. Rev., 1969, 186: 456.

[14] A. J. Leggett, Diatomic molecules and Cooper pairs, in Modern Trends in the Theory of Condensed Matter (SpringerVerlag, Berlin, 1980) pp. 13-27.

[15] P. Nozières and S. Schmitt-Rink, Bose condensation in an attractive fermion gas: From weak to strong coupling superconductivity, J. Low Temp. Phys., 1985, 59: 195.

[16] R. Friedberg and T. D. Lee, Boson-fermion model of superconductivity, Phys. Lett. A, 1989, 138: 423.

[17] T. Friedberg and T. D. Lee, Gap energy and long-range order in the boson-fermion model of superconductivity. Phys. Rev. B, 1989, 40: 6745 .

[18] C. A. R. Sá de Melo, M. Randeria, and J. R. Engelbrecht, Crossover from bcs to bose superconductivity- transition - temperature and time dependent ginzburg-landau theory, Phys. Rev. Lett., 1993, 71: 3202.

[19] M. Randeria, Crossover from BCS theory to Bose-Einstein condensation, in Bose Einstein Condensation, edited by 
A. Griffin, D. Snoke, and S. Stringari (Cambridge Univ. Press, Cambridge, 1995) pp. 355-92.

[20] B. Jankó, J. Maly, and K. Levin, Pseudogap effects induced by resonant pair scattering. Phys. Rev. B, 1997, 56: R11407.

[21] J. Maly, B. Jankó, and K. Levin, Numerical studies of the $s$-wave pseudogap state and related $T_{c}$ : the "pairing approximation" theory, Physica C, 1999, 321: 113.

[22] J. Maly, B. Jankó, and K. Levin, Superconductivity from a pseudogapped normal state: A mode-coupling approach to precursor superconductivity. Phys. Rev. B, 1999, 59: 1354.

[23] Q. J. Chen, I. Kosztin, B. Jankó, and K. Levin, Pairing fluctuation theory of superconducting properties in underdoped to overdoped cuprates. Phys. Rev. Lett., 1998, 81: 4708.

[24] Q. J. Chen, I. Kosztin, B. Jankó, and K. Levin, Superconducting transitions from the pseudogap state: $d$-wave symmetry, lattice, and low-dimensional effects. Phys. Rev. B, 1999, 59: 7083.

[25] I. Kosztin, Q. J. Chen, B. Jankó, and K. Levin, Relationship between the pseudo- and superconducting gaps: Effects of residual pairing correlations below $T_{c}$. Phys. Rev. B, 1998, 58: R5936.

[26] R. Micnas, J. Ranninger, and S. Robaszkiewicz, Superconductivity in narrow-band systems with local nonretarded attractive interactions. Rev. Mod. Phys., 1990, 62: 113.

[27] R. Micnas and S. Robaszkiewicz, Superconductivity in systems with local attractive interactions. Cond. Matt. Phys., 1998, 1: 89.

[28] R. Micnas, M. H. Pedersen, S. Schafroth, T. Schneider, J. Rodriguez-Nunez, and H. Beck, Excitation spectrum of the attractive hubbard model. Phys. Rev. B, 1995, 52: 16223.

[29] J. Ranninger and J. M. Robin, Manifestations of the pseudogap in the boson-fermion model for Bose-Einsteincondensation-driven superconductivity. Phys. Rev. B, 1996, 53: R11961.

[30] M. Drechsler and W. Zwerger, Crossover from BCS superconductivity to Bose condensation, Ann. Physik, 1992, 1: 15.

[31] R. Haussmann, Crossover from BCS superconductivity to Bose-Einstein condensation: a self-consistent theory. Z. Phys. B, 1993, 91: 291.

[32] R. Haussmann, Properties of a Fermi liquid at the superfluid transition in the crossover region between BCS superconductivity and Bose-Einstein condensation, Phys. Rev. B, 1994, 49: 12975.

[33] O. Tchernyshyov, Noninteracting Cooper pairs inside a pseudogap. Phys. Rev. B, 1997, 56: 3372.

[34] E. V. Gorbar, V. M. Loktev, and S. G. Sharapov, Crossover from BCS to composite-boson (local-pair) superconductivity in quasi-2D systems, Physica C, 1996, 257: 355.

[35] V. P. Gusynin, V. M. Loktev, and S. G. Sharapov, Phase diagram of a $2 \mathrm{D}$ metal system with a variable number of carriers, JETP Lett., 1997, 65: 182.

[36] M. Marini, F. Pistolesi, and G. C. Strinati, Evolution from BCS superconductivity to Bose condensation: Analytic results for the crossover in three dimensions, Eur. Phys. J. B, 1998, 1: 151 .

[37] B. DeMarco and D. S. Jin, Onset of fermi degeneracy in a trapped atomic gas, Science, 1999, 285: 1703.

[38] M. H. Anderson, J. R. Ensher, M. R. Matthews, C. E. Wieman, and E. A. Cornell, Observation of Bose-Einstein condensation in a dilute atomic vapor, Science, 1995, 269: 198.

[39] C. C. Bradley, C. A. Sackett, J. J. Tollett, and R. G. Hulet, Evidence of bose-einstein condensation in an atomic gas with attractive interactions, Phys. Rev. Lett., 1995, 75: 1687.
[40] C. C. Bradley, C. A. Sackett, J. J. Tollett, and R. G. Hulet, Evidence of Bose-Einstein condensation in an atomic gas with attractive interactions [Phys. Rev. Lett. 75, 1687 (1995)], Phys. Rev. Lett., 1997, 79: 1170.

[41] K. B. Davis, M. O. Mewes, M. R. Andrews, N. J. van Druten, D. S. Durfee, D. M. Kurn, and W. Ketterle, Bose-Einstein condensation in a gas of sodium atoms, Phys. Rev. Lett., 1995, 75: 3969.

[42] M. Greiner, C. A. Regal, and D. S. Jin, Emergence of a molecular Bose-Einstein condensate from a Fermi gas, $\mathrm{Na}$ ture, 2003, 426: 537.

[43] S. Jochim, M. Bartenstein, A. Altmeyer, G. Hendl, S. Riedl, C. Chin, J. H. Denschlag, and R. Grimm, Bose-Einstein condensation of molecules, Science, 2003, 302: 2101.

[44] M. W. Zwierlein et al., Observation of Bose-Einstein condensation of molecules, Phys. Rev. Lett., 2003, 91: 250401.

[45] C. A. Regal, M. Greiner, and D. S. Jin, Observation of resonance condensation of fermionic atom pairs, Phys. Rev. Lett., 2004, 92: 040403.

[46] M. Bartenstein, A. Altmeyer, S. Riedl, S. Jochim, C. Chin, J. H. Denschlag, and R. Grimm, Crossover from a molecular Bose-Einstein condensate to a degenerate Fermi gas. Phys. Rev. Lett., 2004, 92: 120401.

[47] C. Chin, M. Bartenstein, A. Altmeyer, S. Riedl, S. Jochim, J. Hecker-Denschlag, and R. Grimm, Observation of the pairing gap in a strongly interacting Fermi gas superfluids, Science, 2004, 305: 1128.

[48] M. W. Zwierlein, C. A. Stan, C. H. Schunck, S. M. F. Raupach, A. J. Kerman, and W. Ketterle, Condensation of pairs of fermionic atoms near a Feshbach resonance. Phys. Rev. Lett., 2004, 92: 120403.

[49] J. Kinast, A. Turlapov, J. E. Thomas, Q. J. Chen, J. Stajic, and K. Levin, Heat capacity of a strongly-interacting Fermi gas, Science, 2005, 307: 1296, published online 27 January 2005; doi:10.1126/science.1109220.

[50] M. W. Zwierlein, J. R. Abo-Shaeer, A. Schirotzek, and W. Ketterle, Vortices and superfluidity in a strongly interacting Fermi gas, Nature, 2005, 435: 170404.

[51] M. W. Zwierlein, A. Schirotzek, C. H. Schunck, and W. Ketterle, Fermionic superfluidity with imbalanced spin populations, Science, 2006, 311: 492.

[52] G. B. Partridge, W. Li, R. I. Kamar, Y. A. Liao, and R. G. Hulet, Pairing and phase separation in a polarized Fermi gas, Science, 2006, 311: 503.

[53] P. Fulde and R. A. Ferrell, Superconductivity in a strong spinexchange field, Phys. Rev., 1964, 135: A550.

[54] A. I. Larkin and Y. N. Ovchinnikov, Neodnorodnoe sostoyanie sverkhprovodnikov, Zh. Eksp. Teor. Fiz., 1964, 47: 1136.

[55] A. I. Larkin and Y. N. Ovchinnikov, Inhomogeneous state of superconductors, Sov. Phys. JETP, 1965, 20: 762.

[56] Q. J. Chen, Generalization of BCS theory to short coherence length superconductors: A BCS-Bose-Einstein crossover scenario, Ph.D. thesis, University of Chicago, 2000,, (available in the ProQuest Dissertations \& Theses Database online).

[57] Q. J. Chen, J. Stajic, and K. Levin, Applying BCS-BEC crossover theory to high temperature superconductors and ultracold atomic fermi gases, Low Temp. Phys., 2006, 32: 406, Fiz. Nizk. Temp., 2006, 32: 538.

[58] S. Giorgini, L. P. Pitaevskii, and S. Stringari, Theory of ultracold atomic Fermi gases, Rev. Mod. Phys., 2008, 80: 1215.

[59] C. Chin, R. Grimm, P. Julienne, and E. Tiesinga, Feshbach resonances in ultracold gases, Rev. Mod. Phys., 2010, 82: 1225 . 
[60] M. Inguscio, W. Ketterle, and C. Salomon, eds., Ultracold Fermi gases, Proceedings of the International School of Physics “Enrico Fermi,", Vol. CLXIV, Varenna, 2006, Società Italiana di Fisica Bologna, Italy (ISO Press, Amsterdam, 2008).

[61] H. Ding, T. Yokoya, J. C. Campuzano, T. Takahashi, M. Randeria, M. R. Norman, T. Mochiku, K. Hadowaki, and J. Giapintzakis, Spectroscopic evidence for a pseudogap in the normal state of underdoped high- $T_{c}$ superconductors. Nature, 1996, 382: 51.

[62] C. Renner, B. Revaz, K. Kadowaki, I. Maggio-Aprile, and O. Fischer, Observation of the low temperature pseudogap in the vortex cores of $\mathrm{Bi}_{2} \mathrm{Sr}_{2} \mathrm{CaCu}_{2} \mathrm{O}_{8+\delta}$. Phys. Rev. Lett., 1998, 80: 3606 .

[63] C. Renner, B. Revaz, J.-Y. Genoud, K. Kadowaki, and O. Fischer, Pseudogap precursor of the superconducting gap in under- and overdoped $\mathrm{Bi}_{2} \mathrm{Sr}_{2} \mathrm{CaCu}_{2} \mathrm{O}_{8+\delta}$. Phys. Rev. Lett., 1998, 80: 149.

[64] V. M. Krasnov, A. Yurgens, D. Winkler, P. Delsing, and T. Claeson, Evidence for coexistence of the superconducting gap and the pseudogap in $\mathrm{Bi}-2212$ from intrinsic tunneling spectroscopy, Phys. Rev. Lett., 2000, 84: 5860.

[65] M. Kugler, O. Fischer, C. Renner, S. Ono, and Y. Ando, Scanning tunneling spectroscopy of $\mathrm{Bi}_{2} \mathrm{Sr}_{2} \mathrm{CuO}_{6+\delta}$ : New evidence for the common origion of the pseudogap and superconductivity. Phys. Rev. Lett., 2001, 86: 4911.

[66] J. W. Loram, K. Mirza, J. Cooper, W. Liang, and J. Wade, Electronic specific heat of $\mathrm{YBa}_{2} \mathrm{Cu}_{3} \mathrm{O}_{6+x}$ from 1.8 to $300 \mathrm{~K}$. J. Supercond., 1994, 7: 243.

[67] G. V. M. Williams, E. M. Haines, and J. L. Tallon, Pair breaking in the presence of a normal-state pseudogap in high- $T_{c}$ cuprates, Phys. Rev. B, 1998, 57: 146.

[68] D. J. C. Walker, A. P. Mackenzie, and J. R. Cooper, Transport properties of zinc-doped $\mathrm{YB}_{2} \mathrm{Cu}_{3} \mathrm{O}_{7-\delta}$ thin films, Phys. Rev. $B, 1995,51: 15653$.

[69] T. Graf, J. M. Lawrence, M. F. Hundley, J. D. Thompson, A. Lacerda, E. Haanappel, M. S. Torikachcili, Z. Fisk, and P. C. Canfield, Resistivity, magnetization, and specific heat of $\mathrm{YbAgCu}_{4}$ in high magnetic fields. Phys. Rev. B, 1995, 51: 15053.

[70] Y. F. Yan, P. Matl, J. M. Harris, and N. P. Ong, Negative magnetoresistance in the $c$-axis resistivity of $\mathrm{Bi}_{2} \mathrm{Sr}_{2} \mathrm{CaCu}_{2} \mathrm{O}_{8+\delta}$ and $\mathrm{YBa}_{2} \mathrm{Cu}_{3} \mathrm{O}_{6+x}$, Phys. Rev. B, 1995, 52: R751.

[71] G. Williams, J. L. Tallon, R. Dupree, and R. Michalak, Transport and NMR studies of the effect of Ni substitution on superconductivity and the normal-state pseudogap in $\mathrm{YBa}_{2} \mathrm{Cu}_{4} \mathrm{O}_{8}$, Phys. Rev. B, 1996, 54: 9532.

[72] G. Williams, J. L. Tallon, E. M. Haines, R. Michalak, and R. Dupree, NMR evidence for a $d$-wave normal-state pseudogap. Phys. Rev. Lett., 1997, 78: 721.

[73] K. Magishi, Y. Kituoka, G.-Q. Zheng, K. Asayama, T. Kondo, Y. Shimakawa, T. Manako, and Y. Kubo, Spin-gap behavior in underdoped $\mathrm{TlSr}_{2}\left(\mathrm{Lu}_{0.7} \mathrm{Ca}_{0.3}\right) \mathrm{Cu}_{2} \mathrm{O}_{y}:{ }^{63} \mathrm{Cu}$ and ${ }^{205} \mathrm{Tl}$ NMR studies. Phys. Rev. B, 1996, 54: 3070.

[74] A. Goto, H. Yasuoka, K. Otzschi, and Y. Ueda, Phase diagram for the spin pseudogap in $\mathrm{LaBa}_{2} \mathrm{Cu}_{3} \mathrm{O}_{y}$ studied by NMR, Phys. Rev. B, 1997, 55: 12736.

[75] J. Bobroff, H. Alloul, P. Mendels, V. Viallet, J.-F. Marucco, and D. Colson, ${ }^{17} \mathrm{O}$ NMR evidence for a pseudogap in the monolayer $\mathrm{HgBa}_{2} \mathrm{CuO}_{4+\delta}$, Phys. Rev. Lett., 1997, 78: 3757.

[76] K. Ishida, K. Yoshida, T. Mito, Y. Tokumaga, Y. Kitaoka, K. Asayama, Y. Nakayama, J. Shimoyama, and K. Kishio, Pseudogap behavior in single-crystal $\mathrm{Bi}_{2} \mathrm{Sr}_{2} \mathrm{CaCu}_{2} \mathrm{O}_{8+\delta}$ probed by Cu NMR, Phys. Rev. B, 1998, 58: R5960.
[77] A. V. Puchkov, B. D. N, and T. T, The pseudogap state in high $T_{c}$ superconductors: An infrared study. J. of Phys. Cond. Matter, 1996, 8: 10049.

[78] D. N. Basov, R. X. Liang, B. Dabrowski, D. A. Bonn, W. N. Hardy, and T. Timusk, Pseudogap and charge dynamics in $\mathrm{CuO}_{2}$ planes in YBCO, Phys. Rev. Lett., 1996, 77: 4090.

[79] D. N. Basov, C. C. Homes, E. Singley, M. Strongin, T. Timusk, G. Blumberg, and D. van der Marel, Unconventional energetics of the pseudogap state and superconducting state in high$T_{c}$ cuprates, Phys. Rev. B, 2001, 63: 134514.

[80] J. M. Tranquada, P. M. Gehring, G. Shirane, S. Shamoto, and M. Sato, Neutron-scattering study of the dynamical spin susceptibility in $\mathrm{YBa}_{2} \mathrm{Cu}_{3} \mathrm{O}_{6.6}$. Phys. Rev. B, 1992, 46: 5561.

[81] P. C. Dai, H. A. Mook, S. M. Hayden, and F. Dogan, The connection between superconducting phase correlations and spin excitations in $\mathrm{YBa}_{2} \mathrm{Cu}_{3} \mathrm{O}_{6.6}$ : A magnetic field study, Nature, 2000, 406: 965.

[82] B. Lake, G. Aeppli, T. E. Mason, A. Schroeder, D. F. McMorrow, K. Lefmann, M. Isshiki, M. Nohara, H. Takagi, and S. M. Hayden, Spin gap and magnetic coherence in a clean high-temperature superconductor, Nature, 1999, 400: 43.

[83] G. Ruani and P. Ricci, Transitions at $T>T_{c}$ in underdoped crystals of $\mathrm{YBa}_{2} \mathrm{Cu}_{3} \mathrm{O}_{7-x}$ observed by resonant Raman scattering, Phys. Rev. B, 1997, 55: 93.

[84] X. K. Chen, J. G. Nacini, K. C. Hewitt, J. C. Irwin, R. Liang, and W. N. Hardy, Electronic Raman scattering in underdoped $\mathrm{YBa}_{2} \mathrm{Cu}_{3} \mathrm{O}_{6.5}$, Phys. Rev. B, 1997, 56: R513.

[85] R. Nemetschek, M. Opel, C. Hoffmann, P. F. Muller, R. Hackl, H. Berger, L. Forro, A. Er, and E. Walker, Pseudogap and superconducting gap in the electronic Raman spectra of underdoped cuprates. Phys. Rev. Lett., 1997, 78: 4837.

[86] J. W. Quilty, H. J. Trodahl, and D. M. Pooke, Electronic raman scattering from $\mathrm{Bi}_{2} \mathrm{Sr}_{2} \mathrm{CaCu}_{2} \mathrm{O}_{8+\delta}$ : Doping dependence of the pseudogap and anomalous $600 \mathrm{~cm}^{-1}$ peak. Phys. Rev. $B, 1998$, 57: R11097.

[87] Z. A. Xu, N. Ong, Y. Want, T. Kakeshita, and S. Uchida, Vortex-like excitations and the onset of superconducting phase fluctuation in underdoped $\mathrm{La}_{2-x} \mathrm{Sr}_{x} \mathrm{CuO}_{4}$, Nature, 2000, 406: 486.

[88] Y. Wang, Z. A. Xu, T. Kakeshita, S. Uchida, and N. P. Ong, Onset of the vortexlike Nernst signal above $T_{c}$ in $\mathrm{LaSrCuO}$ and BISrLaCuO, Phys. Rev. B, 2001, 64: 224519.

[89] Y. Y. Wang, N. P. Ong, Z. A. Xu, T. Kakeshita, S. Uchida, D. Bonn, R. Liang, and W. Hardy, The high-field phase diagram of the cuprates derived from the Nernst effect. Phys. Rev. Lett., 2002, 88: 257003.

[90] S. Tan and K. Levin, Nernst effect and anomalous transport in cuprates: A preformed-pair alternative to the vortex scenario, Phys. Rev. B, 2004, 69: 064510.

[91] A. G. Loeser, Z.-X. Shen, D. S. Dessau, D. S. Marshall, C. H. Park, P. Fournier, and A. Kapitulnik, Excitation gap in the normal state of underdoped $\mathrm{Bi}_{2} \mathrm{Sr}_{2} \mathrm{CaCu}_{2} \mathrm{O}_{8+\delta}$. Science, 1996, 273: 325.

[92] A. Kanigel, U. Chatterjee, M. Randeria, M. R. Norman, G. Koren, K. Kadawaki, and J. C. Campuzano, Evidence for pairing above the transition temperature in the electronic dispersion of the pseudogap phase, Phys. Rev. Lett., 2008, 101: 137002.

[93] For simplicity, here we do not discuss electron doping, which is rather similar. Further information can be found in Ref. [7].

[94] S. Chakravarty, R. B. Laughlin, D. K. Morr, and C. Nayak, Hidden order in cuprates, Phys. Rev. B, 2001, 63: 094503.

[95] P. A. Lee, High $T_{c}$ superconductors as doped mott insulators: Fluctuating current and spin chirality, Physica C, 2000, 341- 
348: 63.

[96] P. A. Lee and X.-G. Wen, Vortex structure in underdoped cuprates, Phys. Rev. B, 2001, 63: 224517.

[97] C. Honerkamp and P. A. Lee, Staggered flux fluctuations and the quasiparticle scattering rate in the $\mathrm{SU}(2)$ gauge theory of the $t-J$ model, Phys. Rev. Lett., 2003, 90: 246402.

[98] C. M. Varma, Non-fermi-liquid states and pairing instability of a general model of copper oxide metals, Phys. Rev. B, 1997, 55: 14554.

[99] C. M. Varma, Theory of the pseudogap state of the cuprates, Phys. Rev. B, 2006, 73: 155113.

[100] J. W. Loram, K. A. Mirza, J. R. Cooper, and J. L. Tallon, Specific heat evidence on the normal state pseudogap, J. Phys. Chem. Solids, 1998, 59: 2091.

[101] J. L. Tallon and J. W. Loram, The doping dependence of $T^{*}$ - What is the real high- $T_{c}$ phase diagram? Physica $C, 2001$, 349: 53.

[102] Q. J. Chen, K. Levin, and I. Kosztin, Superconducting phase coherence in the presence of a pseudogap: Relation to specific heat, tunneling, and vortex core spectroscopies. Phys. Rev. B, 2001, 63: 184519 .

[103] P. W. Anderson, The resonating valence bond state in $\mathrm{La}_{2} \mathrm{CuO}_{4}$ and superconductivity, Science, 1987, 235: 1196.

[104] P. W. Anderson, P. A. Lee, M. Randeria, T. M. Rice, N. Trivedi, and F. C. Zhang, The physics behind hightemperature superconducting cuprates: The "plain vanilla" version of RVB, J. Phys. - Condens. Matter, 2004, 16: R755.

[105] N. Nagaosa and P. A. Lee, Ginzburg-landau theory of the spincharge-separated system, Phys. Rev. B, 1992, 45: 966.

[106] For a review of spin-charge separation, see P. A. Lee, Physica C, 1999, 317-318: 194.

[107] Y. J. Uemura, G. M. Luke, B. J. Sternlieb, J. H. Brewer, J. F. Carolan, W. N. Hardy, R. Kadono, J. R. Kempton, R. F. Kiefl, S. R. Kreitzman, P. Mulhern, T. M. Riseman, D. L. Williams, B. X. Yang, S. Uchida, H. Takagi, J. Gopalakrishnan, A. W. Sleight, M. A. Subramanian, C. L. Chien, M. Z. Cieplak, G. Xiao, V. Y. Lee, B. W. Statt, C. E. Stronach, W. J. Kossler, and X. H. Yu, Universal correlations between $T_{c}$ and $n_{s} / m^{*}$ (carrier density over effective mass) in high- $T_{c}$ cuprate superconductors, Phys. Rev. Lett., 1989, 62: 2317.

[108] Y. J. Uemura, Bose-Einstein to BCS crossover picture for high- $T_{c}$ cuprates. Physica C, 1997, 282-287: 194.

[109] V. Mishra, U. Chatterjee, J. C. Campuzano, and M. R. Norman, Effect of the pseudogap on the transition temperature in the cuprates and implications for its origin, Nat. Phys., 2014, 10: 357.

[110] V. J. Emery and S. A. Kivelson, Importance of phase fluctuations in superconductors with small superfluid density. $\mathrm{Na}$ ture, 1995, 374: 434.

[111] M. Franz, Z. Tesanovic, and O. Vafek, QED3 theory of pairing pseudogap in cuprates: From $d$-wave superconductor to antiferromagnet via 'algebraic' Fermi liquid, Phys. Rev. B, 2002, 66: 054535.

[112] I. Ussishkin, S. L. Sondhi, and D. A. Huse, Gaussian superconducting fluctuations, thermal transport, and the nernst effect, Phys. Rev. Lett., 2002, 89: 287001.

[113] J. N. Milstein, S. J. J. M. F. Kokkelmans, and M. J. Holland, Resonance theory of the crossover from Bardeen-CooperSchrieffer superfluidity to Bose-Einstein condensation in a dilute Fermi gas, Phys. Rev. A, 2002, 66: 043604.

[114] Y. Ohashi and A. Griffin, BCS-BEC crossover in a gas of fermi atoms with a feshbach resonance, Phys. Rev. Lett., 2002, 89: 130402 .
[115] N. Andrenacci, P. Pieri, and G. C. Strinati, Evolution from BCS superconductivity to Bose Einstein condensation: Current correlation function in the broken symmetry phase, Phys. Rev. B, 2003, 68: 144507.

[116] A. Perali, P. Pieri, L. Pisani, and G. C. Strinati, BCS-BEC crossover at finite temperature for superfluid trapped Fermi atoms, Phys. Rev. Lett., 2004, 92: 220404.

[117] H. Hu, P. D. Drummond, and X. J. Liu, Universal thermodynamics of strongly interacting Fermi gases, Nat. Phys., 2007, 3: 469.

[118] K. Levin, Q. J. Chen, Y. He, and C.-C. Chien, Comparison of different pairing fluctuation approaches to BCS-BEC crossover, Ann. Phys., 2010, 325: 233.

[119] N. E. Bickers, D. J. Scalapino, and S. R. White, Conserving approximations for strongly correlated electron systems: Bethe-Salpeter equation and dynamics for the two dimensional Hubbard model, Phys. Rev. Lett., 1989, 62: 961.

[120] N. E. Bickers and D. J. Scalapino, Conserving approximations for strongly fluctuating electron systems (I): Formalism and calculational approach, Ann. Phys., 1989, 193: 206.

[121] R. Haussmann, W. Rantner, S. Cerrito, and W. Zwerger, Thermodynamics of the BCS-BEC crossover, Phys. Rev. A, 2007, 75: 023610.

[122] Y. O. Ryota Watanabe, Shunji Tsuchiya, Superfluid density of states and pseudogap phenomenon in the BCS-BEC crossover regime of a superfluid Fermi gas, Phys. Rev. A, 2010, 82: 043630.

[123] P. Magierski, G. Wlazlowski, A. Bulgac, and J. E. Drut, The finite temperature pairing gap of a unitary Fermi gas by quantum Monte Carlo calculations, Phys. Rev. Lett., 2009, 103: 210403.

[124] P. Pieri, A. Perali, G. C. Strinati, S. Riedl, M. J. Wright, A. Altmeyer, C. Kohstall, E. R. S. Guajardo, J. H. Denschlag, and R. Grimm, Pairing-gap, pseudo-gap, and no-gap phases in the radio-frequency spectra of a trapped unitary ${ }^{6} \mathrm{Li}$ gas, Phys. Rev. A, 2011, 84: 011608(R).

[125] L. P. Kadanoff and P. C. Martin, Theory of many-particle systems. II. Superconductivity. Phys. Rev., 1961, 124: 670.

[126] J. Stajic, J. N. Milstein, Q. J. Chen, M. L. Chiofalo, M. J. Holland, and K. Levin, The nature of superfluidity in ultracold Fermi gases near Feshbach resonances, Phys. Rev. A, 2004, 69: 063610.

[127] While a general interaction $V\left(\mathbf{k}-\mathbf{k}^{\prime}\right)$ may not be separable, it can however be decomposed into different channels as $V\left(\mathbf{k}-\mathbf{k}^{\prime}\right)=\sum_{l} \varphi_{\mathbf{k}}^{l} \varphi_{\mathbf{k}^{\prime}}^{l}$, where $\varphi_{\mathbf{k}}^{l}$ represents $s^{-}, p$-, $d$-wave channels, etc. In most cases, only one channel dominates the superfluid order so that we may neglect other channels. In this way, the use of a separable potential is justified.

[128] S. J. J. M. F. Kokkelmans, J. N. Milstein, M. L. Chiofalo, R. Walser, and M. J. Holland, Resonance superfluidity: Renormalization of resonance scattering theory, Phys. Rev. A, 2002, 65: 053617.

[129] Here we will mainly discuss $s$-wave short range contact potential for atomic Fermi gases. At present, $p$-wave superfluids are not yet available experimentally in atomic Fermi gases.

[130] H. Guo, C.-C. Chien, Q. J. Chen, Y. He, and K. Levin, Finite-temperature behavior of an interspecies fermionic superfluid with population imbalance, Phys. Rev. A, 2009, 80: 011601(R).

[131] J. B. Wang, Y. M. Che, L. F. Zhang, and Q. J. Chen, Searching for the elusive exotic Fulde-Ferrell-Larkin-Ovchinnikov states in Fermi-Fermi mixtures of ultracold quantum gases, E-print arXiv:1404.5696. 
[132] C. C. Chien, Q. J. Chen, Y. He, and K. Levin, Intermediate temperature superfluidity in a Fermi gas with population imbalance, Phys. Rev. Lett., 2006, 97: 090402.

[133] C. C. Chien, Q. J. Chen, Y. He, and K. Levin, Superfluid phase diagrams of trapped Fermi gases with population imbalance, Phys. Rev. Lett., 2007, 98: 110404.

[134] Q. J. Chen, Y. He, C.-C. Chien, and K. Levin, Theory of superfluids with population imbalance: Finite temperature and BCS-BEC crossover effects, Phys. Rev. B, 2007, 75: 014521.

[135] J. B. Wang, H. Guo, and Q. J. Chen, Phase diagrams of Fermi gases in a trap with mass and population imbalances at finite temperature, Phys. Rev. A, 2013, 87: 041601(R).

[136] K. M. O’Hara, S. L. Hemmer, M. E. Gehm, S. R. Granade, and J. E. Thomas, Observation of a strongly interacting degenerate Fermi gas of atoms, Science, 2002, 298: 2179.

[137] T. Bourdel, L. Khaykovich, J. Cubizolles, J. Zhang, F. Chevy, M. Teichmann, L. Tarruell, S. J. Kokkelmans, and C. Salomon, Experimental study of the BCS-BEC crossover region in lithium-6, Phys. Rev. Lett., 2004, 93: 050401.

[138] J. Carlson, S. Chang, V. Pandharipande, and K. Schmidt, Superfluid fermi gases with large scattering length, Phys. Rev. Lett., 2003, 91: 050401.

[139] I. Kosztin, Q. J. Chen, Y.-J. Kao, and K. Levin, Pair excitations, collective modes and gauge invariance in the BCS - Bose-Einstein crossover scenario, Phys. Rev. B, 2000, 61: 11662.

[140] Q. J. Chen, Y. He, C.-C. Chien, and K. Levin, Stability conditions and phase diagrams for two-component Fermi gases with population imbalance, Phys. Rev. A, 2006, 74: 063603.

[141] In fact, the parameter $\gamma$ can be taken from experiment, as has been done in Ref. [102], where one can find more details.

[142] P. Pieri, L. Pisani, and G. C. Strinati, BCS-BEC crossover at finite temperature in the broken-symmetry phase, Phys. Rev. B, 2004, 70: 094508 .

[143] N. Fukushima, Y. Ohashi, E. Taylor, and A. Griffin, Superfluid density and condensate fraction in the BCS-BEC crossover regime at finite temperatures, Phys. Rev. A, 2007, 75: 033609 .

[144] I. Kosztin and A. J. Leggett, Nonlocal effects on the magnetic penetration depth in $d$-wave superconductors, Phys. Rev. Lett., 1997, 79: 135.

[145] S. Hufner, M. A. Hossain, A. Damascelli, and G. Sawatzky, Two gaps make a high-temperature superconductor? Rep. Prog. Phys., 2008, 71: 062501.

[146] G. Baskaran, Z. Zou, and P. W. Anderson, Solid State Commun. 63, 973 (1987).

[147] N. Miyakawa, J. Zasadzinski, L. Ozyuzer, P. Guptasarma, D. Hinks, C. Kendziora, and K. Gray, Predominantly superconducting origin of large energy gaps in underdoped BISCO from tunneling spectroscopy, Phys. Rev. Lett., 1999, 83: 1018.

[148] T.-L. Ho, Universal thermodynamics of degenerate quantum gases in the unitary limit, Phys. Rev. Lett., 2004, 92: 090402.

[149] M. L. Chiofalo, S. J. J. M. F. Kokkelmans, J. N. Milstein, and M. J. Holland, Signatures of resonance superfluidity in a quantum Fermi gas, Phys. Rev. Lett., 2002, 88: 090402.

[150] Note here that the definition for $n_{c}$ and $n_{p}$ differ from that in Ref. [156] by a factor of 2 .

[151] G. E. Astrakharchik, J. Boronat, J. Casulleras, and S. Giorgini, Momentum distribution and condensate fraction of a fermion gas in the BCS-BEC crossover, Phys. Rev. Lett., 2005, 95: 230405 , their result seems to suggest a tendency of decrease in the condensate fraction with an increasing particle number used for simulation.
[152] The curves in Fig. 19 were calculated using a two-channel model. Nevertheless, for wide Feshbach resonances such as in ${ }^{6} \mathrm{Li}$ and ${ }^{40} \mathrm{~K}$, the closed-channel fraction is very small $[191,192]$ so that the quantitative difference in the entropy $s(r)$ between the two-channel and one-channel model is negligible.

[153] Q. J. Chen, J. Stajic, and K. Levin, Thermodynamics of interacting fermions in atomic traps, Phys. Rev. Lett., 2005, 95: 260405.

[154] Q. J. Chen, C. A. Regal, M. Greiner, D. S. Jin, and K. Levin, Understanding the superfluid phase diagram in trapped Fermi gases, Phys. Rev. A, 2006, 73: 041601(R).

[155] Note that the experimental data cannot be measuring $N_{c} / N$ as shown in Fig. 18, since at $1 / k_{F} a=-1, N_{c} / N$ is far below the experimental threshold of detection.

[156] J. Stajic, Q. J. Chen, and K. Levin, Density profiles of strongly interacting trapped Fermi gases, Phys. Rev. Lett., 2005, 94: 060401.

[157] While one may argue that the kink, if it exists, may be smoothed out by the $\int \mathrm{d} y \mathrm{~d} z$ integration, we note that as of the time of this writing, no kink behavior has ever been reported in 3D density profiles obtained via an inverse Abel transformation of experimental data.

[158] Q. J. Chen, C. A. Regal, D. S. Jin, and K. Levin, Finite temperature momentum distribution of a trapped Fermi gas, Phys. Rev. A, 2006, 74: 011601(R).

[159] Q. J. Chen, Y. He, C.-C. Chien, and K. Levin, Theory of radio frequency spectroscopy in ultracold Fermi gases and their relation to photoemission in the cuprates, Rep. Prog. Phys., 2009, 72: 122501.

[160] C. H. Schunck, Y. Shin, A. Schirotzek, M. W. Zwierlein, and W. Ketterle, Determination of the fermion pair size in a resonantly interacting superfluid, Nature (London), 2008, 454: 739.

[161] C. H. Schunck, Y. Shin, A. Schirotzek, M. W. Zwierlein, and W. Ketterle, Pairing without superfluidity: The ground state of an imbalanced Fermi mixture, Science, 2007, 316: 867.

[162] Z. H. Yu and G. Baym, Spin-correlation functions in ultracold paired atomic-fermion systems: Sum rules, self-consistent approximations, and mean fields, Phys. Rev. A, 2006, 73: 063601.

[163] G. Baym, C. J. Pethick, Z. H. Yu, and M. W. Zwierlein, Coherence and clock shifts in ultracold Fermi gases with resonant interactions, Phys. Rev. Lett., 2007, 99: 190407.

[164] M. Punk and W. Zwerger, Theory of rf-spectroscopy of strongly interacting fermions, Phys. Rev. Lett., 2007, 99: 170404

[165] A. Perali, P. Pieri, and G. C. Strinati, Competition between final-state and pairing gap effects in the radio-frequency spectra of ultracold Fermi atoms, Phys. Rev. Lett., 2008, 100: 010402 .

[166] S. Basu and E. Mueller, Final-state effects in the radio frequency spectrum of strongly interacting fermions, Phys. Rev. Lett., 2008, 101: 060405.

[167] Y. He, C. C. Chien, Q. J. Chen, and K. Levin, Temperature and final state effects in radio frequency spectroscopy experiments on atomic Fermi gases, Phys. Rev. Lett., 2009, 102: 020402.

[168] M. J. H. Ku, A. T. Sommer, L. W. Cheuk, and M. W. Zwierlein, Revealing the superfluid lambda transition in the universal thermodynamics of a unitary Fermi gas, Science, 2012, 335: 563.

[169] E. Burovski, N. Prokof'ev, B. Svistunov, and M. Troyer, Critical temperature and thermodynamics of attractive fermions at 
unitarity, Phys. Rev. Lett., 2006, 96: 160402.

[170] E. Burovski, E. Kozik, N. Prokof'ev, B. Svistunov, and M. Troyer, Critical temperature curve in BEC-BCS crossover, Phys. Rev. Lett., 2008, 101: 090402.

[171] O. Goulko and M. Wingate, Thermodynamics of balanced and slightly spin-imbalanced Fermi gases at unitarity, Phys. Rev. A, 2010, 82: 053621.

[172] J. Kinnunen, M. Rodriguez, and P. Törmä, Pairing gap and in-gap excitations in trapped fermionic superfluids, Science, 2004, 305: 1131.

[173] Y. He, Q. J. Chen, and K. Levin, Radio-frequency spectroscopy and the pairing gap in trapped Fermi gases, Phys. Rev. A, 2005, 72: 011602(R).

[174] P. Massignan, G. M. Bruun, and H. T. C. Stoof, Twin peaks in rf spectra of Fermi gases at unitarity, Phys. Rev. A, 2008, 77: 031601(R).

[175] J. T. Stewart, J. P. Gaebler, and D. S. Jin, Using photoemission spectroscopy to probe a strongly interacting Fermi gas, Nature (London), 2008, 454: 744.

[176] Q. J. Chen and K. Levin, Momentum resolved radio frequency spectroscopy in trapped Fermi gases, Phys. Rev. Lett., 2009, 102: 190402.

[177] D.S. Jin, private communications; D.S. Jin, American Physical Society March Meeting Talk B8.00002, 2009, abstract available at http://meetings.aps.org/link/BAPS.2009.MAR.B8.2.

[178] J. P. Gaebler, J. T. Stewart, T. E. Drake, D. S. Jin, A. Perali, P. Pieri, and G. C. Strinati, Observation of pseudogap behaviour in a strongly interacting Fermi gas, Nat. Phys., 2010, 6: 569.

[179] A. Perali, F. Palestini, P. Pieri, G. C. Strinati, J. T. Stewart, J. P. Gaebler, T. E. Drake, and D. S. Jin, Evolution of the normal state of a strongly interacting Fermi gas from a pseudogap phase to a molecular Bose gas, Phys. Rev. Lett., 2011, 106: 060402.

[180] A. Perali, P. Pieri, G. C. Strinati, and C. Castellani, Pseudogap and spectral function from superconducting fluctuations to the bosonic limit, Phys. Rev. B, 2002, 66: 024510.

[181] P. Pieri, L. Pisani, and G. C. Strinati, Pairing fluctuation effects on the single-particle spectra for the superconducting state, Phys. Rev. Lett., 2004, 92: 110401.

[182] Y. Shin, M. W. Zwierlein, C. H. Schunck, A. Schirotzek, and W. Ketterle, Observation of phase separation in a strongly interacting imbalanced Fermi gas, Phys. Rev. Lett., 2006, 97: 030401 .

[183] S. Nascimbène, N. Navon, K. Jiang, F. Chevy, and C. Salomon, Exploring the thermodynamics of a universal Fermi gas, Nature (London), 2010, 463: 1057.

[184] S. Nascimbène, N. Navon, S. Pilati, F. Chevy, S. Giorgini, A. Georges, and C. Salomon, Fermi-liquid behavior of the normal phase of a strongly interacting gas of cold atoms, Phys. Rev. Lett., 2011, 106: 215303.

[185] L. P. Gorkov and T. K. Melik-Barkhudarov, Contribution to the theory of superfluidity in an imperfect Fermi gas,, Sov. Phys. JETP, 1961, 13: 1018.

[186] H. Heiselberg, C. J. Pethick, H. Smith, and L. Viverit, Influence of induced interactions on the superfluid transition in dilute Fermi gases, Phys. Rev. Lett., 2000, 85: 2418.

[187] D.-H. Kim, P. Torma, and J.-P. Martikainen, Induced interactions for ultracold Fermi gases in optical lattices, Phys. Rev. Lett., 2009, 102: 245301.

[188] J.-P. Martikainen, J. J. Kinnunen, P. Torma, and C. J. Pethick, Induced interactions and the superfluid transition temperature in a three-component Fermi gas, Phys. Rev. Lett., 2009, 103: 260403.

[189] Z.-Q. Yu, K. Huang, and L. Yin, Induced interaction in a Fermi gas with a BEC-BCS crossover, Phys. Rev. A, 2009, 79: 053636.

[190] Q. J. Chen, Effect of the particle-hole channel on BCS-BoseEinstein condensation crossover in atomic Fermi gases, Eprint arXiv:1109.2307.

[191] Q. J. Chen and K. Levin, Population of closed-channel molecules in trapped Fermi gases with broad Feshbach resonances, Phys. Rev. Lett., 2005, 95: 260406.

[192] G. B. Partridge, K. E. Strecker, R. I. Kamar, M. W. Jack, and R. G. Hulet, Molecular probe of pairing in the BEC-BCS crossover, Phys. Rev. Lett., 2005, 95: 020404.

[193] H. Guo, C.-C. Chien, and K. Levin, Establishing the presence of coherence in atomic Fermi superfluids: Spin-flip and spinpreserving Bragg scattering at finite temperatures, Phys. Rev. Lett., 2010, 105: 120401.

[194] M. G. Lingham, K. Fenech, S. Hoinka, and C. J. Vale, Local observation of pair condensation in a Fermi gas at unitarity, Phys. Rev. Lett., 2014, 112: 100404. 